University of Rhode Island

DigitalCommons@URI

Open Access Master's Theses

1978

\title{
TRICYCLIC ANTIDEPRESSANTS AS UPTAKE BLOCKERS
}

Girolamo A. Ortolano

University of Rhode Island

Follow this and additional works at: https://digitalcommons.uri.edu/theses

\section{Recommended Citation}

Ortolano, Girolamo A., "TRICYCLIC ANTIDEPRESSANTS AS UPTAKE BLOCKERS" (1978). Open Access Master's Theses. Paper 215.

https://digitalcommons.uri.edu/theses/215

This Thesis is brought to you for free and open access by DigitalCommons@URI. It has been accepted for inclusion in Open Access Master's Theses by an authorized administrator of DigitalCommons@URI. For more information, please contact digitalcommons-group@uri.edu. 


\section{TRICYCLIC ANTIDEPRESSAITS \\ AS \\ UPTAKE BIOCKERS}

A THESIS SUBHITTED IN PARTIAL FULFILLMENT OF THE

REQUIREIENTS FOR TEE DFGREE OF

IIASTER OF SCIENCE

IN

PHARMACOLOGY

UNIVERSITY OT RHODE ISLAND

1978 


\section{MASTER OF SCIEHCE THISIS \\ $\mathrm{OF}$ \\ GIROLAMO A. ORTOLANO}

\section{Approved:}

Thesis Committee

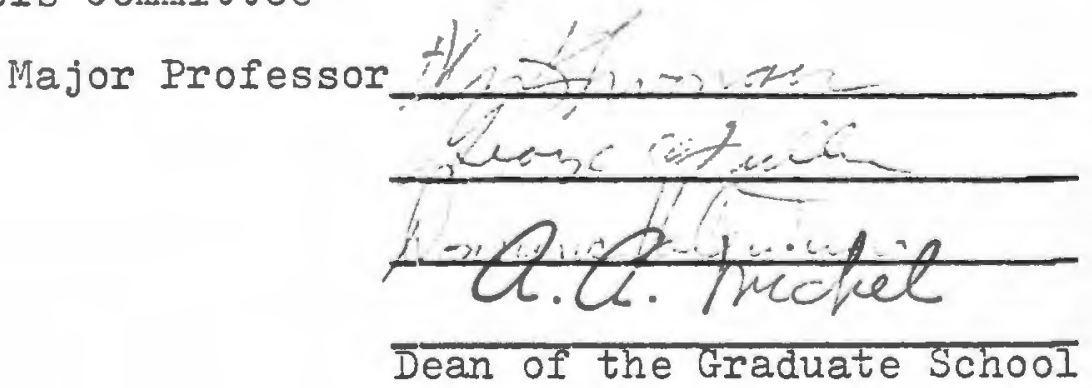

UNIVERSITY OF RHODE ISLAND

1978

ii 


\section{ABSTRACT}

The biogenic amine hypothesis of affective disorders ascribes a functional depletion of neurotransmitters within critical regions of the brain as either the cause or, at the very least, a concomitancy of the depressive syndrome. Much indirect evidence has accummulated to suggest that the therapeutic effect of tricyclic antidepressants (TCA's) is mediated by their ability to block the re-uptake of physiologically released neurotransmitter leaving more neurotransmitter available within the synapse to interact with the post-synaptic receptor, thereby correcting the posited functional depletion.

The evidence upon which the proposed mechanism of TCA's therapeutic activity is based is largely indirect. Most of the evidence comes from in-vitro studies of the ability of tricyclics to block neurotransmitter uptake into either brain slices, minces or preparations of pinched-off nerve endings (synaptosomes). The therapeutic response, however, cannot be demonstrated upon acute administration of these drugs and clinically effective activity is usually not observed until after several weeks of chemotherapy. The uptake inhibitory characteristics which have been demonstrated in-vitro, therefore, cannot explain the latency to the therapeutic effect.

A time-course for the effect of protriptyline administration upon $\mathrm{H}^{3}$-l-norepinephrine $\left(\mathrm{H}^{3}-\mathrm{l}-\mathrm{NE}\right)$ uptake into hypothalamic crude synaptosomal homogenate (CSH) was determined 
after acute, sub-acute and chronic treatment. In addition, the ability of a number of TCA's to block the uptake of three biogenic amines, the catecholamines, norepinephrine and dopamine, and the indoleamine, serotonin, was assessed using a CSH in-vitro test system.

The results support the biogenic amine hypothesis as well as the proposed mechanism of action of TCA's. Uptake blockade could be demonstrated after acute and chronic administration but not after sub-acute administration. An attempt to describe some of the characteristic effects of individual TCA's as well as effects common to the class of drugs was approached on the basis of predominant neurotransmitter influences. 


\section{Acknowledgement}

The traditional acknowledgement must be reserved for those who make the traditional sojourn through the educational process - for me the experience has been anything but traditional. The time invested, the financial and emotional cost represented an insurmountable barrier and was overcome only with the encouragement and assistance of many people. To all of them I offer my sincerest appreciation and mention them here in chronological order.

Thanks to my Mom and Da.d for knowing not to push me into anything and always being willing to pull me out of troubleit's because of them I took the chances I did.

Thanks to my brother Ien for being the model person I chose to emulate, for his support and the fine example he set through. the earliest and perhaps most important years of my life.

Thanks to my long-time Iriend Peter J. Terrara who provided encouragement and the inmeasurabley valuable attitude that anything can be done if you want to do it bad enough.

Thanks to Arnie Ieitner who made me transform thinking about what I wanted to do into doing what I wanted to do.

Thanks to Bob and Delores Olander who provided continual encouragement and the necessary good times.

Thanks to Dr. Gary P. Carlson for the encougaging attitude he expressed in the early years of my interest in graduate work at U.R.I.

Thanks to my friend and advisor Al Swonger who continues to set an example of a unique combination of superior investigative intellect and deep inter- and intra-personal communicative prowess.

Thanks to the State of Rhode Island for their financial support and the faculty of U.R.I. for their understanding during my most difficult periods.

Thanks to Henry Pedro for providing me with much of the necessary inspiration.

Thanks to Ray Scienzo for his long hours of help in setting up the original experimental protocol and for introducing me to H.P.

Thanks to the best family a man could hope to have - my sons Brett and Keith who almost always respected my right to privacy and my need for their good behavior during the long hours I studied in their presence and worked in the lab in 
their absence.

Thanks to Kathryn E. Valeo, a woman like no other more a mother to my sons than most, more a friend to me than móst, an excellent secretary, an excellent lab technician, an unbelieveable person from whom I must continually fight to keep from taking all she has to give.

Thank you, all.

In addition, I would like to acknowledge the unfailing assistance of Roberta Doran and Vicki Burnett at the U.R.I. inter-library loan office for all their help and to i.I. ivadylis Wood who so graciously consented to do the figures in this thesis. 
For Mom, Dad, Brett, Keith and Kathy

vii 


\section{TABLE OP CONTEYTS}

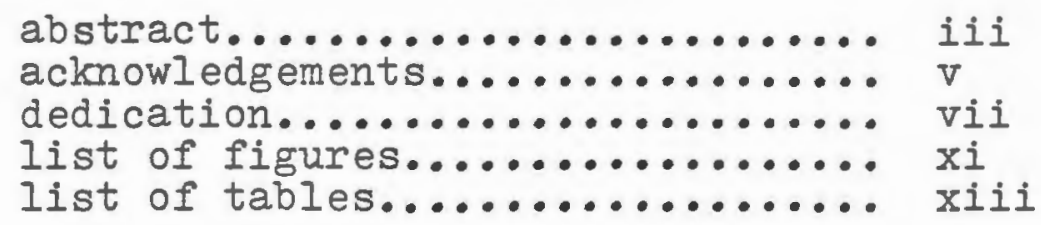

CHAPTER

TITLE

PAGE

INTPODUCTION

A. Neurotransmitter inactivation by uptake processes: An historical background

B. Characterization of kinetically distinct neuronal uptake mechanisms

1. Norepinephrine, dopamine and tyrosine

ii. Serotonin and tryotophan

iii. Other neurohumors

a. Acetylcholine and choline

b. gamma-Aminobutyric acid

c. Glutamate, aspartate and glycine

d. Histamine and histadine

C. Effects of tricyclic antidepressants upon the uptake process.

i. In-vitro

ii. In-vivo

D. Effects of tricyclics on the metabolism of biogenic amines

i. Serotonin

ii. Norepinephrine

iii. Dopamine

III. EXPERIMTITAL WORK

A. Introduction

B. liethods 


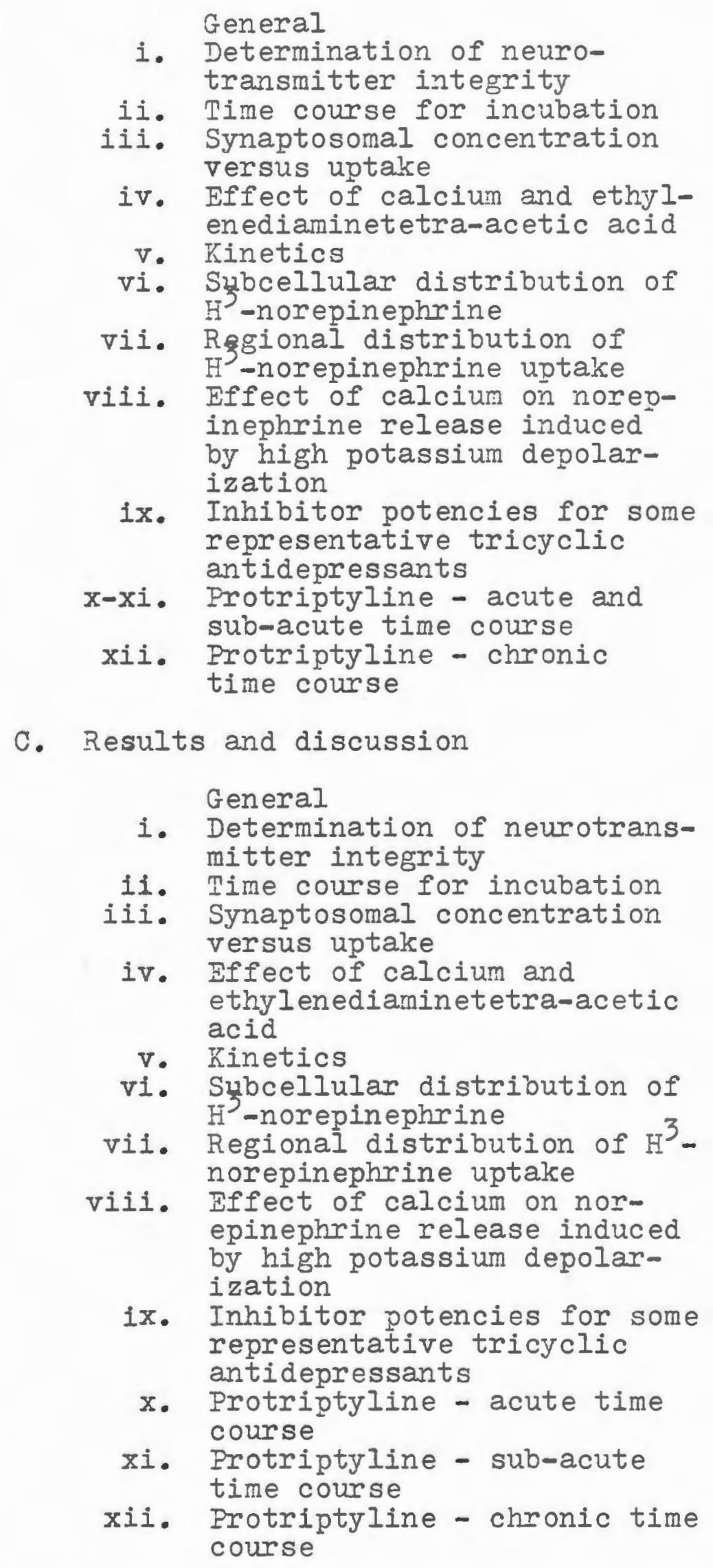


$\begin{array}{ll}\text { IV. } & 102\end{array}$

A. Interpretive constraints 103

3. Altemative mechanisms 106

C. Conclusions 114

V. REFEREMCES 116

$\begin{array}{ll}\text { APPENDIX } & 137\end{array}$ 


\section{IIST OF FIGURES}

1 Functional relationship between brain tissue wet weight and resultant protein concentration in the medium.

2 Integrity of radioactive neurotransmitter substrates determined by thin layer chroma-

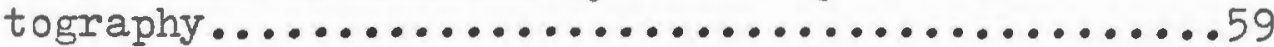

3 Effect of incubation time upon the uptake of E3-norepinephrine into hypothalamic crude synaptosomal homogenate.................61

4 Effect of synaptosomal concentration in the incubation medium upon the uptake of $\mathrm{H}^{3}$-norepinephrine into hypothalamic crude synaptosomal homogenate.......................64

5 Iffect of altering calcium concentrations in the incubation media upon $\mathrm{U} 3$-norevinephrine uptake into hypothalamic crude syñaptosomal homogenate........................67

'Direct Iinear Plot' of $\mathrm{H}^{3}$-norepinephrine uptake into hypothalamic crude synaptosomal

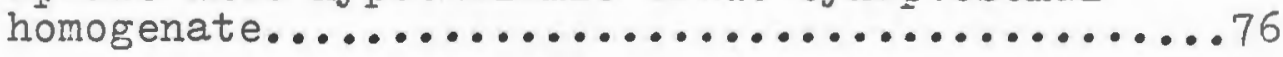

7 Subcellular distribution of previously accumulated $\mathrm{H}^{3}$-norepinephrine into hypothalamic crude synaptosomal homogenate..............81

8 Regional distribution of high affinity

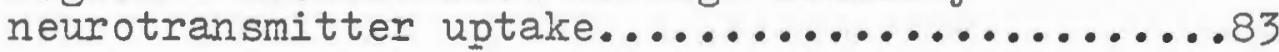

9 Graphical determination of $\mathrm{IC}_{50}{ }^{\prime} \mathrm{s} . \ldots . . . . . . . .88$

10 Time course for the acute administration of protriptyline upon the uptake of $\mathrm{H}^{3}$-norepinephrine into hypothalamic crude synaptosomal homogenate.....................994

11 Time course for the subacute administration of protriptyline upon the uptake of $\mathrm{E}^{3}$-norepinephrine into hypothalamic crude synapto-

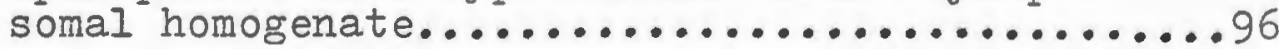

12 Time course for the chronic administration of protriptyline upon the uptake of $\mathrm{H}^{3}$-norepinephrine into hypothalamic crude synapto-

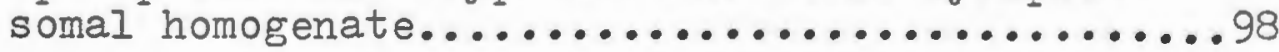




\section{IIST OF FIGURES (continued)}

No.

Title

Page

A-1 Flowchart for 'VIVO': A program used to calculate data for synaptosomal uptake

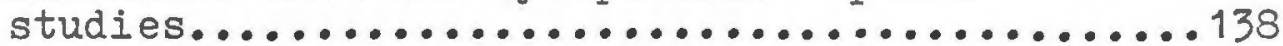




\section{LIST OF TABLES}

1 Reproducibility of the brain dissection

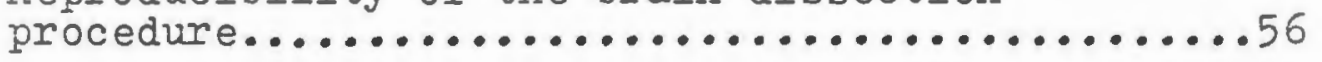

2 Comparison of methods used to determine kinetics coistants for $\mathrm{H}^{3}$-norepinephrine uptake into rat hypothalamic crude synaptosomal homogenate......74

3 Kinetics constants obtained for norepinephrine uptake using substrate concentrations in the

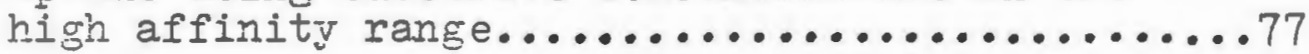

4 Kinetics constants obtained for serotonin uptake using substrate concentrations in the ingh

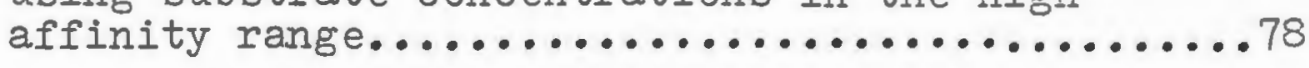

5 Kinetics constants obtained for dopamine uptake using substrate concentrations in the high affinity range.

6 Effects of high potassium depolarization pretreatment upon subsequently measured in-norepinephrine uptake.

7 Norepinephrine uptake $I_{50}$ 's for representative secondary and tertiary amines of the tricyclic antidepressant class of drugs................90

8 Serotonin uptake $I_{50}$ 's for representative secondary and tertiary amines of the tricyclic antidepressant class of drugs.................91

9 Dopamine uptake $I_{50}$ 's for representative secondary and tertiary amines of the tricyclic antidepressant class of drugs................92

10 Summary of relative and absolute potencies of representative secondary and tertiary amines of the tricyclic antidepressant class of drugs..109

A-1 Iisting of 'VIVO': A program used to calculate data for synaptosomal uptake studies..........139 
CHAPTER I: INTRODUCTION 
The biogenic amine hypothesis of affective disorders, admirably and extensively reviewed by Baldessarini (1975), was first proposed by Jacobsen in 1959 and popularized by Schildkraut and Kety (1967). The hypothesis developed from observations of the effects of drugs upon emotionality and the resultant biochemical changes produced by these drigs in experimental animals. Reserpine, once used widely in the treatment of essential hypertension, produces depression in a significant proportion of patients necessitating withdrawal of the medication. Reserpine has also been shown to drastically deplete levels of physiologically active biogenic neurotransmitter amines within the brains of experimental animals. The biogenic amine hypothesis attributes lowered levels or turnover of these amines as either the cause or, at the very least, a concomitancy of the depressive syndrome. Further support for the hypothesis comes from the noted clinical antidepressant properties of a class of drugs referred to as monoamine oxidase inhibitors (MAOI). Iproniazid, an early member of the class, produced mood elavation in patients being treated for tuberculosis. Inhibition of MAO, an enzyme which degrades biogenic amines within the central nervous system (CNS), results in increased levels of the amines. Reviews of the support for the biogenic amine hypothesis abound (Davis, 1970; Schildkraut, 1973; Leonard, 1975; Weil-Malherbe, 1972) and even some dealing selectively with either norepinephrine (Schildkraut, 1965), dopamine (McClure, 1973) or serotonin (Lapin and Oxenkrug, 1969) as 
the critical neurotransmitter exist.

Tricyclic antidepressants have been shown to be effective inhibitors of the primarily neurotransmitter-specific, "hi-affinity", uptake process which many investigators believe is responsible for the termination of activity of physiological concentrations of neurotransmitter at the synapse. The ability of these antidepressants to block the uptake of neurotransmitter from the synapse may result in more neurotransmitter avallable within the synapse to interact with the post-synaptic receptor. This property of the drug would logically tend to reverse the effect of a lowered level or lowered potency or, in terms of the biogenic amine hypothesis, a functional depletion of neurotransmitter and can account for a reversal of the depressive symptoms.

The onset of clinical antidepressant activity, however, cannot be explained by correlations with antidepressant plasma levels (Oswald, 1972). A lag of several weeks of treatment before antidepressant activity becomes apparent has been reported in clinical trials of tricyclic antidepressant therapy (Hammer, et al., 1962; Asberg et al., 1970). Uptake studies to date deal primarily with in-vitro effects of antidepressants and in doing so cannot uncover any significant alterations in the drug effects on the uptake systems which might be coincident with and possibly related to the clinical antidepressant activity. Although one recent study (Ross and Renyi, 1975b) reports the effect of uptake blockade in animals treated for one day orally, there 
has not been a study reporting the effect of chronic administration of tricyclics on the hi-affinity uptake system of biogenic amines in the central nervous system. The design of many antidepressant drugs and the animal screening models for potential new antidepressants relies heavily upon the uptake inhibitory property of the tricyclics. Although the property of uptake inhibition fits the heuristic biogenic amine hypothesis, it has yet to be established that the therapeutic effect is mediated by this mechanism either entirely or in part.

A test of the hypothesis that tricyclic antidepressants mediate this therapuetic activity by inhibiting the uptake of biogenic amines can be approached experimentally using isolated pinched off nerve endings (synaptosomes) as a model of nerve endings functioning in-situ (Kuhar, 1973; Bradford, 1975). Characterization of the model and optimization of the parameters for unidirectional transport are the required preliminary experiments. The classical pharmacological approach necessitates establishing a time course for the effect as well as a dose-response curve. Treatment effects may then be compared with that of vehicle injected controls to conclude the test of this hypothesis.

To confirm that uptake inhibition occurs upon repeated administration of tricyclics would lend support to the biogenic amine hypothesis and further substantiates the concept that tricyclics may mediate their effect by virtue of this well documented property. 
CHAPTER II: REVIEW OF THE IITERATURE 
A. Neurotransmitter inactivation by uptake processes: An historical background.

Interest in measuring the uptake of catecholamines and the physiological significance of this process came about as a result of a number of experiments designed to illustrate the mechanism by which catecholamines are inactivated under physiological conditions. Monoamine oxidase (MAO) was then considered the enzyme responsible for inactivating the catecholamines at sympathetic nerve endings in much the same way in which acetylcholinesterase was thought to act at cholinergic neurons (Burn, 1953). Koelle and Valk (1954) reported that unlike acetylcholinesterase in cholinergic neurons, MAO is not selectively associated with adrenergic neurons. Kamijo et al. (1955), Balzer and Holtz (1956) and Corne and Graham (1957) all reported the inability of a variety of MAOI's to significantly potentiate the actions of catecholamines either administered exogenously or released by stimulation of the sympathetic nerve.

Catechol-0-methyltransferase (COMT) has been shown to play a role in the metabolism of catecholamines (Axelrod, 1957, 1959; Hertting and Axelrod, 1961; Rosell et al., 1963), but Bacq (1959) reported only a slight potentiation of the effects of endogenous catecholamines produced by stimulation of sympathetic, nerves after COMT was inhibited by pyrogallol. Wylie et al. (1960) reported similar results upon administration of exogenous catecholamines as did Murnaghan and Mazurkiewicz (1963) using another COMT inhibitor, 4-methyltropolone. 
Burn (1932) first suggested that exogenously administered catecholamines might be taken up into storage sited of certain peripheral tissue, but it was not until fifteen years later that Raab and Humphreys (1947) reported an increase in catecholamine content of cat and dog hearts after the administration of large doses of epinephrine and norepinephrine.

Acceptance of the importance of tissue uptake in the inactivation of catecholamines awaited the development of radioactively labeled catecholamines with high specific activity and this uptake was demonstrated by Axelrod et al. (1959). ${ }^{3} \mathrm{H}$-Epinephrine $(0.1 \mathrm{mg} / \mathrm{kg})$ administered to mice was found to disappear in two phases. The first phase was a rapid metabolism of approximately $70 \%$ of the injected dose. The remaining 30\%, however, disappeared more slowly. The interpretation of these results was that exogenously administered catecholamines are accumulated in tissues and may serve to inactivate catecholamines under normal physiological conditions. Whitby et al. (1961) drew the same conclusions from the resilts of similarly designed experiments. Further support for the concept of uptake being responsible for inactivation of catecholamines came from Muscholl (1961) using a combination of bioassay and flourimetric techniques and from the laboratory of stromblad and Nickerson (1961) as well. Numerous reports substantiating this conclusion followed and are reviewed by Iversen (1965). Considerable evidence has accumulated to suggest that 
the uptake of catecholamines occurs into post-ganglionic nerve endings. Kopin et al. (1964) maintained that if allowances are made for differences in regional blood flow through various tissues studied, there is a good correlation between the accumulation of norepinephrine and the level of endogenous norepinephrine. The levels of endogenous norepinephrine may be taken to reflect the extent of innervation of the organ or tissue by sympathetic postganglionic terminals, although turnover may be judged a better indicator by some (Costa, 1969). Additional evidence that the uptake of norepinephrine occurs primarily into sympathetic post-ganlionic neuronal endings became available when Hertting et al. (1961) reported almost complete disappearance of the ability of norepinephrine and epinephrine to accumulate in tissues in which the sympathetic postganglionic nerves innervating the tissue had been cut and the terminals allowed to degenerate. This approach was modified by Zaimis et al. (1965), Iversen et al. (1966) and Sjoqvist et al. (1967) whereby sympathetic innervation was obliterated by immunosympathectomy at birth; the results obtained were similarly interpreted.

A number of techniques have been employed to conclude that localization of accumulated radiolabeled norepinephrine occurs predominantly in neuronal endings include studies using density gradient centrifugation (Potter and Axelrod, 1962; 1963), autoradiography and electron microscopy (Worle et al., 1962; Marks et al., 1962; Samorajski et al., 1964) 
and histochemical flourescence techniques (Hamberger et al., 1964). Although the evidence implicates the neuronal ending as the site of uptake, methodology at that time was not available to accurately separate and describe the component of uptake into tissue other than neuronal endings. Fischer and Kopin (1964), however, did present evidence to indicate that the uptake of catecholamines was not limited to neuronal tissue in their report describing uptake into denervated salivary glands of the rat.

Further elaboration of the characteristics of uptake systems of peripheral tissues came about as a result of attempts to study the effects of drugs. Macmillan (1959) proposed the "nerve uptake hypothesis" ascribing the potentiating properties of cocaine to its ability to interfere with the catecholamine uptake mechanism. Adrenergic blocking agents were found to inhibit the uptake of ${ }^{3} \mathrm{H}-$ norepinephrine including phenoxybenzamine and dicholroisoproterenol (Axelrod et al., 1962b). Tricyclic antidepressants, neuroleptics of the phenothiazine class as well as guenethidine, bretylium and ouabain were shown to be effective inhibitors of the noradrenergic uptake mechanism (Axelrod et al., 1961b; Herhitting et al., 1962; Dengler et al., 1962) as well as the sympathomimetic amines tyramine, amphetamine and ephedrine (Axelrod and Tomchick, 1960; Axelrod et al., 1961a, Heritting et al., 1962; Dengler et al. 1961).

Muscholl (1960), Axelrod et al. (1961b) and Herltting 
et al. (1962) all reported that reserpinized tissues failed to accumulate epinephrine and norepinephrine. Dengler (1961) found that the accumulation of epinephrine and norepinephrine in tissue slices was prevented when reserpine was present in the incubation medium. As a result of these experiments there was relatively wide acceptance that reserpine inhibited the uptake of catecholamines (Brodie and Beaven, 1963). After an extensive review of the literature, however, Shore (1963) concluded that the actions of reserpine were predominantly centered around the intracellular uptake and storage mechanism and not the neuronal membrane uptake system. Direct evidence to support this hypothesis came from Iindmar and Muscholl (1964) where in both normal and reserpinized isolated rat heart preparations, they infused $0.5 \mathrm{ug}$ of norepinephrine over a 10 minute period and collected and analyzed the perfusate for unchanged norepinephrine. Analyzing the norepinephrine content of the heart after the perfusion interval, they determined that in normal hearts the catecholamine taken up from the medium was almost entirely accounted for as unchanged norepinephrine accumulated into the tissue. In reserpinized hearts, however, the amount of norepinephrine in the perfusate was the same as in the perfusate of normal hearts, but tissue analysis revealed very much lower levels of catecholamine with very high levels of MAO metabolites in the perfusate. These These results suggested that norepinephrine accumulation was 
not affected but its subsequent storage was.

Iversen (1965a, 1965b) reported characteristics of the uptake of norepinephrine into isolated rat hearts. At low concentrations of epinephrine or norepinephrine (.07- $7 \mathrm{uM}$ ) the uptake followed classical Michaelis-Menten kinetics and $\mathrm{Km}$ and $V_{\max }$ values where calculated showing a greater affinity of the stereoisomer, l-norepinephrine, for the uptake mechanism than d-norepinephrine. The racemate, dl-norepinephrine, exhibited a higher $V_{\max }$ and lower $\mathrm{Km}$ than dl-epinephrine.

Similar experiments were performed using high concentrations of catecholamines (5-230 uM) in the perfusion medium (Iversen 1965a, 1965b). The accumulation of catecholamines under these conditions could not be explained in terms of the uptake system described when using low catecholamine concentrations in the perfusate, however, the characteristics of this uptake process could be described using classical enzyme kinetic models. This second uptake system, a process for which the term Uptake $2\left(\mathrm{U}_{2}\right)$ has been applied, showed a much lower affinity than could be ascribed to the initial uptake system described (referred to as Uptake 1, abbreviated $\mathrm{U}_{1}$ ).

Several properties of $\mathrm{U}_{2}$ serve to distinguish it from $\mathrm{U}_{1}$. Catecholamines accumulated by $\mathrm{U}_{2}$ disappeared rapidly from the tissue if the perfusion was continued using a catecholamine-free medium, although 2 ug. of catecholamine per gram of tissue remained even after prolonged periods of 
washout. A comparison of the kinetic constants showed that unlike $U_{1}, U_{2}$ exhibited no stereospecificity; also, although $\mathrm{U}_{2}$ accumulated norepinephrine more rapidly, epinephrine exhibited a greater affinity for uptake with the Km being about five times lower than that for norepinephrine. Although $\mathrm{U}_{2}$ appeared inoperative at low substrate concentrations and thought to be activated where a critical threshold of substrate concentration is reached (Iversen, 1965a), a more recent study reported evidence to conclude that both systems operate at all substrate concentrations with $U_{l}$ having the greater influence at low and $\mathrm{U}_{2}$ at high substrate concentrations (Iightman and Iversen, 1969).

Another property serving to distinguish between $U_{1}$ and $\mathrm{U}_{2}$ includes substrate specificity and the effect of drugs on inhibiting the transport effected by each system. Burgen and Iversen (1965) reported the results of a variety of substituted $B$-phenylethylamines on the $\mathrm{U}_{2}$ system. Interestingly, O-methylation as well as $\mathbb{N}$ - substitution greatly enhanced that affinity for $\mathrm{U}_{2}$; wherea's the effect of these compounds on $U_{1}$ are exactly opposite in nature (Horn, 1973). Cocaine, imipramine and desipramine while potent inhibitors of $\mathrm{U}_{1}$ were found to be only weakly active with respect to $U_{2}$ (Iversen 1965a; Salt, 1972). Phenoxybenzamine is a potent inhibitor of $\mathrm{J}_{2}$ as is a variety of steroids including B-estradiol, corticosterone, testerone, deoxycorticosterone and androsterone (Iversen and Salt, 1972).

Originally it was suggested that $\mathrm{U}_{2}$ might represent a 
separate neuronal membrane transport system at sites along the entire neuron; that is, cell body, axon and nerve terminal. Evidence for this concept was provided by the histochemical studies of Norbert and Hamberger (1964) and Hamberger et al. (1964) who reported that the accumulation of norepinephrine was localized to terminal regions of postganglionic sympathetic neurons when low doses of norepinephrine were administered and amine throughout the cell body and axon as well as the terminals when high doses were administered. This hypothesis, however failed to explain the washout phenomenon reported to exist for $\mathrm{U}_{2}$. Another possibility offered was that $U_{2}$ may represent an uptake of catecholamines by storage granules within adrenergic neuronal terminals (Iversen, 1965a). Evidence for this hypothesis included the indisputable similarity of properties between $U_{2}$ and either isolated adrenergic nerve granules or adrenal medullary granules incubated in-vitro (Euler and Iishajko, 1964; Carlsson et al., 1963). The particle uptake system is saturable at amine concentrations of $80 \mathrm{ug} / \mathrm{ml}$. which is very close to that described for $U_{2}$, also, both are relatively insensitive to inhibition by cocaine, desipramine or metraminol and both are inhibited by tyramine and pkienoxybenzamine. Granular uptake lacks specificity for the optical isomers of epinephrine and norepinephrine. Finally, the uptake of catecholamines in isolated granules occurs at concentrations of $1 \mathrm{ug} / \mathrm{ml}$. or higher in the absence of magnesium and adenosine triphos- 
phate and $U_{2}$ is known to begin to exert substantial influence at this concentration of amine. One important difference between $\mathrm{U}_{2}$ and the isolated particles is reflected by the fact that no difference in affinity can be shown for epinephrine and norepinephrine as can be observed in the $\mathrm{U}_{2}$ system.

The hypothesis which has gathered the most support by far is that $U_{2}$ represents an extra-neuronal uptake mechanism (Iversen, 1975). Support has come from the results of histochemical studies of the distribution of catecholamines in various tissues after exposure to high concentrations of exogenously administered norepinephrine. The work of Fisher and Kopin (1964) cited earlier may have provided that needed impetus to investigate the association between non-neuronal or extra-neuronal uptake and $\mathrm{U}_{2}$. After perfusion of rat hearts with medium containing high concentrations of norepinephrine, intense flourescence was observed in cardiac muscle cells and unidentified connective tissue cells as well as nerve terminals (Ehinger and Sporrong, 1968; Farnebo and Maimfors, 1969; Clarke et al., 1969; Jacobowitz and Brus, 1971). Extraneuronal uptake, like $\mathrm{U}_{2}$, was found to be sensitive to normetanephrine and relatively insentive to desimpramine (Farnebo and Malfors, 1969; Clarke et al., 1969). Numerous reports of uptake of catecholamines into nonneuronal tissue including salivary gland parenchyma (Hamberger, 1967), smooth muscle of cat spleen (Gillespie 1968, 1973), vas deferens (Gillespie et al., 1970), chick 
amnion (Burnstock et al., 1971), perfused rabbit ear artery (Avakian and Cillespie, 1968), cat nictitating membrane (Draskoczy and Trendlenburg, 1970) and guinea pig trachea (O'Donnell and Saar, 1973), appeared. Kinetic constants for the extra-neuronal uptake into arterial cells was shown to have characteristics similar to $\mathrm{U}_{2}$ (Gillespie and Towart, 1973). The uptake of norepinephrine into smooth muscle also showed susceptibility to phenoxybenzamine and washout was inhibited by normetanephrine (Gillespie, 1968).

Although $U_{1}$ has been considered the principal mechanism responsible for the inactivation of physiological concentrations of norepinephrine (Iversen, 1971), the preponderance of sympathetic innervation is clearly a consideration in determining the importance of either uptake mechanism in isolated tissue preparations, as is the concentration of catecholamine in the medium. Smooth muscle of the vasculature contains relatively little sympathetic innervation and it would seem reasonable to conclude that $U_{2}$ is most important in terminating the action of circulating catecholamines in this tissue. The evidence to support the hypothesis that neuronal uptake is the principal inactivating mechanism of depolarize-released neurotransmitter comes from reports that organ response to sympathetic nerve stimulation is greatly increased by drugs shown to be effective inhibitors of the neuronal uptake mechanism (Thoenen, 1964a, 1964b; Brown, 1965). Based on results reported by Ianger (1970), U $U_{1}$ may account for the recapture of as much as 70- 
$90 \%$ of the transmitter released by low frequency sympathetic nerve stimulation in the cat nictitating membrane. 
B. Characterization of kinetically distinct neuronal uptake mechanisms.

i) Norepinephrine, dopamine and tyrosine:

Norepinephrine uptake as an active process described using Michaelis-Menten kinetics was dissociated from the contribution of passive diffusion by using low substrate concentrations $\left(10^{-7} \mathrm{M}\right.$ ) in the medium of brain slice preparations (Dengler, 1962). The active component of uptake into brain slices was shown to be temperature dependent, occur against a concentration gradient, be dependent upon $\mathrm{Na}^{+}$in the medium, require oxygen and glucose, can be lowered by metabolic inhibitors (Dengler et al., 1962; Dengler, 1965; Ross and Renyi, 1964, 1966), and does not require calcium or magnesium ions (Hamberger, 1967). Limited accumulation occurs when incubation is carried out in a sucrose medium (Mirkin et al., 1963).

Similar results have been reported with the use of a crude synaptosomal homogenate. Davis et al. (1967), Bogdanski and Tissari (1967), Bogdanski et al. (1968) Colburn et al. (1968) and White and Keen (1971) all showed an absolute requirement for $143 \mathrm{mM} \mathrm{Na}^{+}$concentrations and inhibitory effects of increasing $\mathrm{K}^{+}$concentrations above $5 \mathrm{mM}$, as well as temperature dependence and reduction in uptake by metabolic inhibitors. Conclusions of these studies suggest that uptake by brain slices, at low substrate concentrations similar to that used by Dengler (1962), occurs primarily into neuronal endings.

Harris and Baldessarini (1973) showed similar require- 
ments for dopamine uptake into rat corpus striatum with one notable exception. Whereas Snyder and Coyle (1969) reported no substantial effect on initial uptake of catecholamines using reserpine pretreatment, Harris and Baldessarini (1973) reported a considerable effect.

Additional support for the hypothesis that amine accumulation occurs into nerve endings comes from the work of Snyder et al. (1965) who showed that intraventricular injection of labeled norepinephrine was found localized to a great extent in the synaptosomal fraction upon differential centrifugation. Histochemical techniques confirmed that the uptake of monoamines into rat cerebral cortex was confined to varicosities indistinguishable from those containing endogenous norepinephrine (Eamberger and Masuoka, 1965; Hamberger, 1967). Eenn (1967) using electron microscopic autoradiography reported that $80 \%$ of the $\mathrm{H}^{3}$-norepinephrine accumulated by brain slices was localized to neurons. More recently Iidbrink and Jonsson (1974) showed a reduction of uptake of norepinephrine into cerebral cortices after noradrenergic terminals were destroyed by lesion of the dorsomedial reticular formation with 6-hydroxydopamine.

Snyder and Coyle (1969) studied the stereospecificity of norepinephrine and dopamine uptake in different areas of the rat brain. The Michaelis constant of 0.4 uN for norepinephrine uptake into extrastriatal areas is in good agreement with the 0.56 uM $\mathrm{Km}$ reported by Colburm et al. 
(1968) using whole rat brain. Snyder and coyle (1969) extended the range of catecholamine concentration from 0.05 uM using 1.2 uM as the upper limit. They reported that the striatum had a higher affinity for dopamine than norepinephrine and both neurotransmitters display only a single uptake component over the range of substrate concentrations studied. All other brain areas showed two components of dopamine uptake, one considered a high affinity the other a low-affinity transport mechanism, whereas norepinephrine continued to be described adequately by a straight line using the Iineweaver-Burk method suggesting only one transport system exists for this amine. In a later paper (Coyle and Snyder, 1969), they reported the $\mathrm{Ki}$ values for mutual inhibition of dopamine and norepinephrine uptake in all regions of rat brain to be identical with their respective $\mathrm{Km}$ values and concluded that these two catecholamines use the same transport systems (presumably dopaminergic) and would not require such a characteristic.

The uptake of precursors of norepinephrine and dopamine, namely tyrosine and dopa, have been described (Naeme, 1961; Guroff et al., 1961; Dengler et al., 1962; Yoshida et al., 1963a, 1963b, 1963c, 1965) but delineation of two transport systems has not been the focus of attention and whether these systems exist remains to be shown. Chirigos et al., (1960) have reported stereospecificity of 1-tyrosine for brain tissue and illustrated its uptake inhibition by the amino acids leucine, isoleucine, valine, histadine and 
cysteine. They also noted that stereospecificity was lacking in muscle preparations. Interestingly, Ross and Renyi (1966) studied the uptake of tyramineand 1-dopa at concentrations similar to those reported for high affinity uptake of dopamine (Snyder and Coyle, 1969) and reported $\mathrm{Km}$ 's for dopamine and tyrosine of $0.7 \mathrm{uM}$ and $0.4 \mathrm{uM}$, respectively, in slices of whole brain. These values are similar to those reported for extra-striatal norepinephrine uptake (Coyle and Snyder, 1969; Snyder and Coyle, 1969). It would appear that these $\mathrm{Km}$ values adequately describe a high affinity component of uptake when compared with other amino acids with $\mathrm{Km}^{\prime} \mathrm{s}$ in the millimolar range (Cohen and Lajtha, 1972). In addition, Wofsey et al. (1971) present evidence to indicate that tyrosine may become selectively incorporated into a population of synaptosomes that can be separated from those which accumulate norepinephrine by a technique known as incomplete equilibrium centrifugation.

ii) Serotonin and tryptophan:

Fuxe and Ungerstedt (1967) described the localization of intraventricularly administered serotonin to serotonergic neurons using histochemical flourescence techniques. Although a number of attempts were made to illustrate serotonin accumulation in brain slices (for a review see Katz and Chase, 1970) most attempts were unsucessful in separating out an active transport process from simple diffusion until Blackburn et al. (1967) incubated brain slices with $c^{14}$-serotonin at a concentration of $2 \times 10^{-8} M$ and 
reported considerable accumulation of the amine with $60 \%$ recovered in the synaptosomal fraction upon subcellular fractionation using differential centrifugation techniques. Similarly, Kuhar et al. (1970) studied the subcellular localization of serotonin after incubation of brain slices with the amine in concentrations ranging from $10^{-5}$ to $10^{-8} \mathrm{M}$ and found the major portion of accumulated serotonin localized to synaptosomes.

The kinetics of serotonin accumulation into slices of rat striatum and hypothalamus revealed two distinct transport processes with $\mathrm{Km}_{\mathrm{H}}=1.7 \times 10^{-7} \mathrm{M}$ and $\mathrm{Km}_{\mathrm{L}}=8.0 \times 10^{-6} \mathrm{M}$ in the striatum and $\mathrm{Km}_{\mathrm{H}}=1.4 \times 10^{-7} \mathrm{M}$ and $\mathrm{Km}_{\mathrm{L}}=8.0 \times 10^{-6} \mathrm{M}$ in the hypothalamus (Shaskan and Snyder, 1970). The Ki values for the competitive inhibition of norepinephrine uptake by serotonin approximated the $\mathrm{Km}$ for low affinity serotonin into catecholaminergic neurons. These kinetically distinct components were confirmed by Wong et al. (1973).

Kuhar et al. (1974) review the results of a number of studies illustrating the reduction of serotonin uptake associated with the selective destruction of serotonin nerve terminals by midbrain raphe lesions suggesting that uptake occurs predominantly into serotonergic nerve endings when low substrate concentrations are employed. Histoahemical flourescence as well as the measurement of tryptophan hydroxylase activity and endogenous serotonin levels support the hypothesis that high affinity uptake process is specific for neurotransmitter inactivation upon depolarized release 
(Kuhar et al., 1972). Massari and Sanders-Bush (1975) have utilized this concept of high-affinity neurotransmitterspecific uptake to compare the effects of para-choloroamphetamine or B-9 lesions with the distribution of serotonergic neuronal endings in different areas of the brain. Initial studies of tryptophan transport into synaptosomal preparations utilized substrate concentrations limited to the low affinity component of transport (Grahame-Smith and Farfitt, 1970). In this study the concentrations of sodium ions in the media were varied from 0 to $112 \mathrm{mM}$ by osmotic replacement with either choline or sucrose and resulted in no effect on tryptophan uptake, however, cyanide and ouabain did significantly reduce the uptake of substrate. Interestingly, preloading the synaptosomes with l-tryptophan resulted in an increase in the uptake of either 1-tryptophan or 1-phenylalanine. I-Tryptophan preloaded synaptosomes were also shown to greatly accelerate the rate of efflux of tryptophan by either 1-tryptophan or 1-phenylalanine implying that a faciltated exchange process was occuring. A number of amino acids were reported very active in stimulating the efflux of tryptophan from preloaded synaptosomes. It would appear that this low affinity transport system represents the same mechanism by which other amino acids gain entry into the neuron and is not specific for tryptophan.

A more recent study revealed a high affinity component of the uptake of l-tryptophan as well as a low affinity component (Parfitt and Grahame-Smith, 1974). Although the 
affinity Michaelis constant $\left(\mathrm{Km}_{L}\right)$ originally reported by Grahame and Parfitt (1970) as $1.0 \times 10^{-3} \mathrm{M}$ is considerably disparate from their more recent data $\left(\mathrm{Km}_{\mathrm{I}} 3.2 \times 10^{-4} \mathrm{M}\right)$; use of different tissue preparation, i.e. whole brain vs. cerebral cortex, can account for the results. The $\mathrm{Km}_{\mathrm{F}}$, however, is only lower than the the $\mathrm{Km}_{\mathrm{I}}$ by a factor of three reported in this study, (Farfitt \& Grahme-Smith, 1974), whereas, other investigators have reported differences between high and low affinity uptake systems differing from a minimum of 21 (in cerebral cortex) to over 300 (glycine in the spinal cord) orders of magnitude which would appear to represent physiologically more distinguishable processes (Snyder et al., 1973).

\section{iii) Other neurohumors:}

a) Acetylcholine and choline:

Unlike the catecholamines and serotonin, acetylcholine appears to be inactivated largely by the intrasynaptic degradative enzyme acetylcholinesterase and diffusion rather than an uptake mechanism (for a review see Katz and Miledi, 1973). Early work aimed at characterizing an uptake process for acetylcholine did not prove fruitful (Guth, 1962; Burton, 1964). Even in the presence of the reversible cholinesterase inhibitor physostigmine, negative results were reported (Elliot and Henderson, 1951; Schuberth and Sundwall, 1967) although it was suggested that physostigmine may have strong inhibitory actions on the uptake mechanisms (Polak and Meeuws, 1966). Use of the organophosphate cholinester- 
ase inhibitors including paraoxon (Diethyl-4-nitrophenyl phosphate) have resulted in significant accumulations of acetylcholine into brain tissue (Polak and Meeuws, 1966; Schuberth and Sundwall, 1967; Iiang and Quastel 1969; Heilbronn, 1969) with the uptake requirement for oxygen, reduced by metabolic inhibitors and described using Michaelis-Menten kinetics (Schuberth and Sundwall, 1967; Polak and Meeuws, 1966; Liang and Quastel, 1969).

Acetylcholine uptake seems unimportant physiologically for it has been shown that acetylcholine uptake into synaptosomes is neither readily incorporated into vesicles (Marchbanks, 1968) nor released by electrical stimulation or potassium depolarization (Katz et al., 1973). Further evidence leading to a conclusion that acetylcholine uptake is not physiologically important comes from the results of Marchbanks (1969b) and Adamic (1970) suggesting that both molecules utilize the same carrier. Similarly autoradiographic techniques have been employed using slices of rat cortex incubated in labeled acetylcholine and the distribution of label was reported as diffuse with no obvious preference for specific cell types or structures (Polak, 1969). Finally, Katz et al. (1973) attempted to collect the excess acetylcholine which might be produced by brain stimulation in the presence of an acetylcholine uptake inhibitor if the acetylcholine transport process were physlologically active. They report that no additional acetylcholine is collected in the presence of uptake inhibitor 
and conclude that acetylcholine uptake may not be physiologically operational. These results may be explained by considering the transport mechanism responsible for acetylcholine uptake to be identical with the choline carrier; the low affinity choline transport mechanism being common to all cells requiring choline as substrate for synthesis of phasphatidyl- or phosphorycholine.

Hodgkin and Martin (1965) first described the ability of choline to be transported into excitable:tissue, the squid axon. A year later Schuberth et al. (1966) reported the presence of choline in cerebral cortex tissue slices and localization of choline transport within nerve endings was established by Marchbanks (1968b) using synaptosomal preparations with a reported $\mathrm{Km}$ of 100uM. A number of similar studies reported $\mathrm{Km}$ 's varying from as $10 \mathrm{w}$ as $80 \mathrm{uM}$ (Potter, 1968) to 440 uM (Hensworth and Bosmann, 1971).

Green and Haubrich (1971) using synaptosomal preparations incubated with low concentrations of choline, reported a significant amount of acetylcholine formed and suggested that more than one uptake system may exist for choline accumulation in the brain. Yamamura and Snyder (1973) and Dowdall and Simon (1973) reported two kinetically distinct carrier-mediated systems for choline transport having a $\mathrm{Km}_{\mathrm{H}}$ of 1-5 uM and $\mathrm{Im}_{\mathrm{L}}$ less than $25 \mathrm{uM}$. The two systems showed clearly distinguishable effects of $\mathrm{Na}$ concentration (Haga and Noda, 1973) with the high affinity component more dependent on alterations in sodium concentration in the media. 
Although it has been suggested that the high affinity component represents a mechanism of cholinergic neurons which may serve to recapture the product of intrasynaptic acetylcholinesterase for the synthesis, storage and subsequent reutilization of acetylcholine, conclusive evidence of this is not available as yet (Kuhar and Murrin, 1978).

Kuhar, et al. (1973) have provided evidence to indicate that choline uptake occurs into cholinergic neurons primarily by the high affinity uptake process. Septal lesions, known to result in reductions of acetylcholine in the cerebral cortex and hippocampus, resulted in a reduction of choline uptake by $60 \%$ in synaptosomes prepared from these cholinergically innervated areas, whereas, tryptophan and glutamatic acid accumulation were unaffected although GABA and norepinephrine uptake were affected slightly.

b) gamma-Aminobutyric acid (GABA)

GABA was shown to be taken up by slices of rat cerebral cortices when a low concentration ( $0.05 \mathrm{uM}$ ) of substrate was used in the medium (Iversen and Neal, 1963). The electron microscopic autoradiographic experiments of Bloom and Iversen (1971) suggest that more than $70 \%$ of the GABA taken up into brain slices is localized to the nerve terminals. It has also been suggested that at this low concentration, reminiscent of that used to detect high affinity uptake, a distinct population of nerve terminals are responsible for the uptake of labeled GABA (Iversen and Snyder, 1968; Iversen and Johnston, 1971). Clearly distinguishable 
high and low affinity components for GABA uptake into synaptosomes and brain slices have been reported (Ievi and Raiteri, 1973a, 1973b).

As with other high affinity transport systems there is an absolute requirement for physiological concentrations of electrolytes and uptake is inhibited strongly by low concentrations of $\mathrm{Na}^{+}$(Iversen and Neal, 1968). Inhibition of GABA uptake has been reported with 2,4-dinitrophenol, ouabain and malonate (Kuriyama et al., 1969; Snodgrass et al., 1973).

c) Glutamate, aspartate and glycine:

Logan and Snyder (1972), Balcar and Johnston (1972a, 1972b) and Bennett et al. (1972) have studied the uptake of glutamate and aspartate and have reported that transport occurs by both high and low affinity mechanisms with the high affinity system having an absolute dependence on sodium ions. Furthermore, the metabolic requirements of both glutamate and aspartate uptake are indistinguishable with azide, eyanide and omission of glucose from the media having minimal effects and it appears as though glutamate and aspartate share the same transport carrier. Wofsey et al. (1971), using subcellular distribution studies, have associated the high affinity uptake with a unique population of nerve terminals suggesting that the high affinity mechanism may be directly related to the function of replenishment of neurotransmitter.

A high affinity uptake system specific for glycine has 
been shown in slices of rat spinal cord, pons and medulla (Neal, 1971; Johnston and Iversen, 1971), slices of cat spinal cord (Balcar and Johnston, 1973) as well as in crude synaptosomes of rat spinal cord (Logan and Snyder, 1972; Bennett et al., 1972) and in purified synaptosomes of cerebral cortex of the rat (Peterson and Raghupathy, 1973). Iocalization of glycine uptake into nerve endings by the high affinity system has been shown by autoradiographic analysis (Matus and Dennison, 1972; Iversen and Bloom, 1972; Ehinger, 1972; Hosli and Hosli, 1972; Ljungadhl and Hokfelt, 1973) as well as subcellular fractionation techniques (Johnston and Iversen, 1971; Arregui et al., 1972). Glick (1972) has shown inhibitors of $\mathrm{Na}^{+} / \mathrm{K}^{+}$dependent ATP-ase to be effective inhibitors of glycine uptake; however, no inhibitors has yet been described as being more effective in inhibiting either the high or the low affinity uptake. The low affinity mechanism of glycine uptake seems to be relatively non-specific in that 1-alanine, 1-serine and other small neutral amino acids compete for the transport site (Cohen and Iajtha, 1972).

d) Histamine and histadine:

Although the probable mechanism of histamine inactivation is via the degradative enzyme histamine methyltransferase, subcellular distribution studies show the incorporation into the $P_{2}$ (synaptosomal) fractions and after subsequent treatment in a hypo-osmotic medium histamine was shown to be localized to the vesicular fraction (snyder 
et al., 1974). Young et al. (1971) reported an ontogenic related change in the distribution of histamine among subcellular fractions. Older animals (2l days) showed greatest accumulation in the synaptosomal fraction whereas younger animals appeared to concentrate histamine in the nuclear pellet.

A stereospecific uptake of I-histidine into brain slices has been demonstrated (Neame, 1964) although the substrate concentrations were not low enough to differentiate a high affinity component and in all probability the low affinity system is shared by other basic amino acids such as tryptophan. 
C. Effects of tricyclic antidepressants on the hi-affinity uptake process of serotonergic noradrenergic and dopaminergic neurons.

i) in-vitro

The ability of tricyclic antidepressants to block the uptake of norepinephrine into brain slices was described by Dengler et al. (1961) using concentrations of radioactively labeled norepinephrine $\left(2.8-14.0 \times 10^{-8_{M}}\right)$ well within the concentration range where hi-affinity transport is responsible for a large part of the uptake of norepinephrine into nerve endings. The concentration at which the uptake of norepinephrine into nerve endings is inhibited by fifty percent, $\left(I C_{50}\right)$, varies depending upon the preparation used, 1.e. brain slices versus synaptosomes, as well as by brain region. The $I_{50}$ for desmethylimipramine (DMI) varles from 0.003 uM in rat brain synaptosomes (Ross and Renyi, 1975a) to 0.11 uM in rat brain slices (Salama et al., 1971). Buus Iassen et al., (1975) reported an $I_{50}$ for DMI of $10 \mathrm{uM}$ in rat forebrain synaptosomes as opposed to 0.001 uM in synaptosomes prepared from rat hippocampal tissue. The large difference is believed to be due to the concentration of noradrenergic neuronal endings within brain regions (Squires, 1974; Buus Lassen et al., 1975).

It is also true that studies utilizing different species as well as different preparations reported identical $I_{50}$ values for the tricyclics tested. Ross and Renyi (1967a) utilizing mouse cortex slices reported an $I_{50}$ value of 0.03 uM for the inhibition of norepinephrine uptake 
by DMI whereas an identical value was reported by Shaskan and Snyder (1970) using a crude synaptosomal homogenate of rat hypothalamus. A good correlation between the partition coefficient and the $I_{50}$ values for a number of chemically different uptake inhibitors was found to exist in brain slices (Carmichael and Israel, 1973).

All studies report DMI to be the most potent tricyclic tested with respect to its ability to block norepinephrine uptake (Ross and Renyi, 1967a; Shaskan and Snyder, 1970; Horn et al., 1971; Salama et al., 1971; Carmichael and Israel, 1973; Squires, 1974; Maxwell et al., 1974; Ross and Renyi, 1975a; Buus Lassen et al., 1975). Many studies indicate that the demethylated form of the tertiary amines, i.e. the secondary amines, are more potent with respect to their ability to inhibit norepinephrine uptake whereas the tertiary amines are more efficacious in its effect on serotonin uptake (Blackburm et al., 1967; Ross and Renyi 1967b; 1969; Carlsson, 1970; Shaskan and Snyder, 1970; Kannengiesser et al., 1973; Horm and Trace, 1974; Ross and Renyi, 1975a and Buus and Lassen et al., 1975).

Structure activity relationships have been studied with respect to both the noradrenergic (Horn and Snyder, 1972; Ferris et al., 1972; Horn, 1973a; Naxwell et al., 1974) and serotonergic (Horn, 1973b) transport system in the central nervous system. It is on the basis of these studies that evidence can be found to substantiate the concept that there exists a chemically distinguishable transport 
mechanism for each neurotransmitter.

The uptake inhibitory characteristics of tricyclics as they apply to dopamine have been studied (Ross and Renyi, 1967a). Their effect on this transport system has not generally been considered as important due to the relatively large $I C_{50}$ values reported (Horn et al., 1971). There is no correlation with the inhibition of uptake of dopamine into either rat hypothalamus or whole brain synaptosomes with respect to methyl substitution on the side chain nitrogen of any of the tricyclic antidepressants (Horn et al., 1971; Halaris et al., 1975). Alternatively, antiparkinsonian drugs have been shown to be very potent in their ability to block dopamine uptake in the central nervous system (Coyle and Snyder, 1969b).

\section{ii) in-vivo}

Imipramine was shown to reduce the amount of labeled epinephrine found in homogenates of whole mice when compared. with saline treated controls (Axelrod and Tomchick, 1959). Similarly, imipramine increased the rate of disappearance of labeled norepinephrine in the whole animal (Axelrod and Tomchick, 1960). Hertting et al. (1961) reported reduced levels of $\mathrm{H}^{3}$-norepinephrine in heart, spleen, adrenals, liver and muscles with elevated normetanephrine found in muscle tissue after treatment with imipramine. Glowinski and Axelrod (1964) administered imipramine DMI or amitriptyline to rats and after 1 hour administered $\mathrm{H}^{3}$-norepinephrine by an intra-cerebroventricular (ICV) injection. All three 
compounds were reported to be effective in inhibiting the uptake of $\mathrm{H}^{3}-\mathrm{NE}$ into rat brain. It is interesting to note that Schanberg (1963) could not show imipramine to be effective in inhibiting the uptake of 5-hydroxytrptophan, the precursor of serotonin, into brain slices when the drug was administered i.p. I hour prior to sacrifice with subsequent testing in-vitro.

As with the in-vitro data, many studies measuring the effectiveness of tricyclics on either norepinephrine or serotonin uptake report tertiary amines as being more effective then the secondary amines in blocking the uptake of 5HT in the central nervous system (Ross and Renyi, 1975b). Conversely, a number of studies conclude the secondary amines to be more effective than tertiary amines in blocking NE uptake (Ross and Renyi, 1967; Schildkraut et al., 1969a; Carlsson et al., 1969b; Ross and Renyi, 1975b).

Palaic et al., (1967) reported no uptake inhibitory effect of DMI 10mg/kg; injected i.p. 20 minutes prior to ICV $\mathrm{H}^{3}-5-\mathrm{HT}$ injection. Similarly, Carlsson (1970) could not show an effect of DMI on the release of serotonin by H75/12 into rat brain. Schubert et al., (1970) failed to demonstrate an effect on the rate of disappearance of $\mathrm{H}^{3}-5-\mathrm{HT}$ after intravenous injection of $\mathrm{H}^{3}$-tryptophan of either secondary amine, DMI or nortriptyline, whereas both imipramine and amitriptyline retarded the rate of disappearance of $\mathrm{H}^{3}-5-\mathrm{HT}$. Histochemical evidence is available to suggest that imipramine blocks serotonin uptake (Carlsson et al., 1968; Corrodi and Fuxe, 1968). 
D. Effects of tricyclics on the metabolism of biogenic amines:

i) Serotonin

Da Prada and Pletscher (1966) reported a significant reduction in the levels of 5-hydroxyindoleacetic acid, (5-IIAA) the major metabolite of serotonin in the CNS, for both imipramine and amitriptyline. The ratio of product to precursor indicates that imipramine is slightly more efficacious in reducing the turnover of serotonin and this is reflected in the in-vitro data as well (Ross and Renyi, 1969; 1975; Kannengiesser, 1973). Palaic et al., (1967), using an intracerebroventricular perfusion of $\mathrm{H}^{3}-5 \mathrm{HT}$ at 55 uM concentration for 1 hour, demonstrated a reduction in endogenous levels of 5-HIAA but not of $\mathrm{H}^{3}-5 \mathrm{HIAA}$. At this concentration the predominant idoleamine inactivation mechanism is presumably a low affinity transport system. It seems possible that the effect of DMI on inhibiting the uptake of $5 \mathrm{HT}$ may have been overshadowed by the low affinity transport mechanism as well as diffusion. A reduction in endogenous levels of 5HIAA imply decreased activity of the neuron which may come about as a result of feedback mechanisms in the event DMI is inhibiting 5HT uptake. Subsequently increases in the intrasynaptic amine concentration and hence its interaction with post-synaptic receptors is the postulated mechanism for feedback inhibition (Costa and Meek, 1974). $\mathrm{C}^{14}$-Serotonin was administered intracistermally 15 minutes prior to an i.p. injection of either DMI or IMI 
(25mg/kg) and 2 hours after the intracisternal injection brains were assayed for $\mathrm{H}^{3}-5 \mathrm{HT}$ and deaminated metabolites (Schildkraut et al., 1969b). The results indicate imipramine as being more effective in increasing levels of radioactive amine. These results are consistentwith the idea that the tricyclics reduce the turnover of $5 \mathrm{HT}$ by the postulated inhibitory feedback mechanism. Similar results were obtained in the study of Schubert et al., (1970) in which both imipramine and amitriptyline reduced the turnover of $5 \mathrm{HT}$ as measured by a reduction in the ratio of $\mathrm{H}^{3}$ tryptophan to $\mathrm{H}^{3}-5 \mathrm{HT}$. Bruinvels (1972) and Alpers and Himwich (1972) reported reduced turnover of $5 \mathrm{HT}$ as a result of tricyclic treatment. More recently, Ieonard and Kafoe (1976) showed a decreased turnover of serotonin with both acute and chronic imipramine as well as DMI treatments. Buus Lassen et al., (1975) tested the effects of tricyclics administered 3 hours prior to sacrifice and all resulted in lowering levels of 5HIAA.

i1) Norepinephrine

Schanberg et al., (1967) administered either IMI or DMI 90 minutes prior to the intraventricular administration of $\mathrm{H}^{3}-\mathrm{NE}$ and demonstrated increased levels of $\mathrm{H}^{3}$-normetanephrine in the brains of drug treated rats. When the drugs were given 90 minutes after the intraventricular injection of $\mathrm{H}^{3}-\mathrm{NE}$ and animals were sacrificed 3 hours after the antidepressant, the levels of labeled norepinephrine increased. Normetanephrine increased and deaminated 
catechol metabolites decreased reflecting the anticipated response of agents which block the uptake mechanism. Schildkraut et al. (1969a) demonstrated greater decreases in levels of $\mathrm{H}^{3}-\mathrm{NE}$ by treatment with secondary amines DMI and PRO than by the tertiary amines IMI and AMI. In fact AMI did not result in altering the levels of $\mathrm{H}^{3}-\mathrm{NE}$ found in the brains of drug treated rats when compared with saline treated controls. These results are similarly reflected in the in-vitro data of Ross and Renyi (1967a) and Shaskan and Snyder (1970). Schildkraut et al. studied the effects of chronic administration of the tertiary amine, imipramine (1970), and the secondary amine protriptyline (1971), on the levels of norepinephrine and its metabolites. In these experiments, tricyclic antidepressants were administered by intra-peritoneal injection and $\mathrm{H}^{3}-\mathrm{NE}$ followed 6 hours later by intracisternal injection. The animals were sacrificed either 6 minutes or $31 / 2$ hours after the labeled compound was administered. Upon acute administration, imipramine significantly reduced the levels of $\mathrm{H}^{3}-\mathrm{NE}$ when animals were sacrificed 6 minutes after the label whereas the reduction by protriptyline was not statistically significant. Normetanephrine levels were increased whereas deaminated catechols and 0-methylated deaminated metabolites were decreased for both drugs studied. When the animals were sacrificed 3 1/2 hours after the label was administered, a trend towards increased values of norepinephrine was noted but did not achieve statistical significance. These 
results could be explained within the framework of the proposed mechanism of action of these drugs as transport inhibitors. It seems reasonable that after $91 / 2$ hours the drug was nearly completely metabolized and the neurons responded to the effect of decreased levels in the presence of drug to increasing their levels by facilitating the uptake of amines. Normetanephrine levels remained somewhat elevated and deaminated catechol metabolites were not nearly as depressed as after the 6 minute sacrifice period. Chronic administration of either drug resulted in decreased levels of $\mathrm{H}^{3}$-norepinephrine when studied either 6 minutes or $31 / 2$ hours after administration of the labeled amine. Similarly, normetanephrine was increased and deaminated catechol metabolites were severely depressed. The levels of endogenous amines were reduced below control for both drugs. The data suggests that both the secondary and tertiary amines have the same effect on norepinephrine metabolism when administered chronically; that of inhibiting uptake and increasing turmover as measured by the rate of disappearance of $\mathrm{H}^{3}-\mathrm{NE}$ from the brain (levels of $\mathrm{H}^{3}-\mathrm{NE}$ at 6 minutes vs. levels at $31 / 2$ hours after label).

In a more recent study of the effect of chronic administration of IMI and DMI, tumover of the biogenic amines including norepinephrine was reported as being decreased although the decrease was not statistically significant (Leonard and Kafoe, 1976). In these experiments turnover was measured as the ratio of specific activity of labeled 
product to labeled precursor after administration of $\mathrm{H}^{3}$ tyrosine and $\mathrm{H}^{3}$-tryptophan. The results are obviously at variance with that reported by Schildkraut et al. (1970, 1971) with respect to the turnover of norepinephrine, and the authors could not offer a reason for the apparant discrepancy. The effects of chronic administration of tricyclics on the turnover of norepinephrine awaits further study before a conclusion can be drawn.

\section{iii) Dopamine}

Dopamine has been the least studied of the catecholamines with respect to the effects of tricyclic antidepressants on its metabolism. Da Prada and Pletscher (1966) reported a slight but insignificant decrease of homovanillic acid (HVA) after an acute administration of imipramine whereas amitriptyline caused a significant increase in HVA. Similar results were obtained by Nielsen (1975) in a study of the affect of acutely administered amitriptyline. Levels of dopamine were shown to be increased although the increase did not achieve statistical significance. DMI, however, did increase the levels of dopamine significantly.

Chronic administration of both imipramine and DMI decreased the turmover of dopamine (Ieonard and Kafoe, 1976). 
CHAPTER III: EXPERIMENTAI WORK 


\section{A. Introduction:}

The use of any experimental system necessitates an evaluation of its performance characteristics or characterization. The rigor applied to characterization is often goverened by the investigators knowledge and understanding of the system as acquired through a review of the literature and personal experience. In this regard, the following characterization experiments were performed to establish some of the important parameters pertinent to the experimental protocol as well as some characteristics believed to be Indicative of a properly functioning system.

Following the characterization are experiments designed to directly test the hypothesis that protriptyline blocks the uptake of tritiated 1-norepinephrine in an in-vitro hypothalamic synaptosomal test after acute, subacute and chronic administration of the drug. Since the methodology employed in the characterization differs little with that used for the test of the hypotheses, the methods section contains a "general methods" section and the deviations from the general methods are noted in the subsequent numbered subsections corresponding to each individual experiment.

Subsection 1 through ix can be considered characterization experiments and $x$ through $x i 1$ are specifically directed towards the objective of this thesis.

\section{B. Methods:}

General: Male Long Evans rats (Charles River Breeding 
Iabs) weighing 200-450 gms. were group housed with food and water given ad libitum in a windowless room in which an artificial photoperiod was provided (light: 0800-1600 E.S.T.). Animals were sacrificed by decapitation at approximately the same time each day (11:00-12:00 noon) to eliminate the possible source of error attributable to chronobiological variations in the uptake of neurotransmitters as reported by Lew and Quay (1974). The latency from decapitation to immersion of the dissected brain tissue into ice cold $0.32 \mathrm{M}$ sucrose never exceeded 1.0 minute and was usually accomplished within 45 seconds.

Brains were dissected using a modification of the method developed by Glowinski and Iversen (1966). The brain was removed from the skull and placed with its ventral side upon a previously cooled glass plate positioned on ice (generally within 20 seconds from decapitation). An icecold stainless steel spatula was used to make a medialsaggital incision to the level of the corpus callosum. With the lateral ventricles as the starting point the cerebral cortex was rolled back laterally to expose the hemisphere of tissue containing the characteristic striae of the striatum. The medial aspect of the striatal tissue was teased free with the blade of the spatula. After removal of both striatal tissue masses the cortex was folded closed and placed on its dorsal surface. The end of a curved spatula was inserted at the posterior portion of the Circle of Willis and maneuvered in such a way as to emerge at its 
anterior portion. The tissue was dissected free along the side edges of the spatula with a dissecting needle. Although referred to here as hypothalamic this tissue mass is predominantly hypothalamic in origin with some mid-brain tissue as depicted in the diagram provided by Glowinski and Iversen (1966). The remaining tissue was placed on either lateral side and the hemisphere partially separated to observe the approximate location of the genu of the corpus callosum. The brain was then sliced in a coronal plane with the genu as its posterior boundary. This tissue mass was termed forebrain and contained cerebral cortex and some limbic structures.

The dissected tissue was immediately placed into icecold 0.32 sucrose $(10 \% \mathrm{w} / \mathrm{v})$ and allowed to sit on ice until all dissections were completed. The tissue was then homogenized in a Teflon ${ }^{R}$-glass tissue grinder with a clearance of $0.030 \mathrm{~cm}$ using 10 up and down strokes at about 1,000 r.p.m. The supernatant fraction of a $1,000 \times \mathrm{g} \times 10$ minute centrifugation carried out at $4^{\circ} \mathrm{C}$ in a Sorvall RC-2B refrigerated centrifuge was used as the crude synaptosomal homogenate (CSH).

The incubation medium (IM) originally used in preliminary experiments was Kreb's bicarbonate (Umbreit et al., 1964), as modified by snyder and Coyle (1969) and contained in millimoles ( $\mathrm{mM}$ ): $\mathrm{NaCl}(118.5), \mathrm{KCl}(4.8) \mathrm{CaCl}_{2}(1.2)$ $\mathrm{KH}_{2} \mathrm{PO}_{4}(1.2) \mathrm{MgSO}_{4} 7 \mathrm{H}_{2} \mathrm{O}(1.2), \mathrm{NaHCO}_{3}$ (24.8), d-glucose (11.1), ascorbic acid (1.1), disodium ethylenediamine tetra- 
acetic acid (EDTA) (0.13) and the monoamine oxidase inhibitor (MAOI), nialamide $(0.01)$. The ascorbic acid and EDTA were added to increase the stability of the neurotransmitters by preventing auto-oxidation shown to occur in oxygenated media (Iversen, 1972).

One milliliter (ml.) of $\mathrm{CSH}$ was introduced into $6-8 \mathrm{ml}$. of incubation medium (IM) resulting in a concentration of 0.4 to $0.9 \mathrm{mg}$. protein per ml. IM. Protein was determined by the method of Iowry et al. (1951). Usually, six aliquots were equally divided among $0^{\circ} \mathrm{C}$ and $37^{\circ} \mathrm{C}$ conditions. In some experiments the number of replicates per condition reached 8.

The radio-labeled neurotransmitter ( $2 \mathrm{uCi} / \mathrm{ml}$ ) was added to the IM in a volume of 5 microliters (ul.) per ml. of IM resulting in an IM which was 0.1 uM with respect to neurotransmitter. One ml. aliquots (minimum of 3 and maximum of 8 determinations per condition) were incubated at $37^{\circ} \mathrm{C}$ in a Dubnoff metabolic shaker (with no agitation) and an equal number were kept on ice in glass test tubes (10 mm O.D. $\times 75 \mathrm{~mm})$. After incubation for the specified time interval (norepinephrine and dopamine, 10 minutes; serotonin 5 minutes) the tubes were placed into a Sorvall RC-2B refrigerated centrifuge to spin at $13,000 \times \mathrm{g} \times 60$ minutes to form a pellet containing synaptosomes, mitochondria and microsomes or the $\mathrm{P}_{2}$ fraction described by Gray and Whittaker (1962).

One-tenth $\mathrm{ml}$. of the supernatant was placed into 
scintillation vials containing $2 \mathrm{ml}$. of absolute ethanol prior to the addition of toluene phosphor scintillation fluid. Pellets were solubilized in $1.0 \mathrm{ml}$. of absolute ethanol with the aid of a bent dissecting needle used to break up the pellet and the solution was poured into a scintillation vial. The tubes were washed with $1.0 \mathrm{ml}$. of absolute ethanol and this wash was added to the scintillation vial prior to the addition of scintillation fluid.

Ten ml. of toluene phosphor containing $5.5 \mathrm{~g} /$ liter of 2,5-diphenyloxazole (PPO) and $125 \mathrm{mg} / \mathrm{liter}$ of B-bis-(2phenyloxazole)-benzene ( $P O P O P$ ) in toluene. The radioactivity was counted in a Packard Tri-Carb Model 3335 for 10 minutes. The efficiency for tritium was optimum (45\%) at a gain setting of 57.5 with the window virtually wide open. Quenching between samples was not found to be significant and therefore no correction was made. Background counts was subtracted from all samples with counts less than ten times the value obtained for background.

The data are expressed as either Tissue (or Particulate): Medium ratios or uptake in nanograms/mg. protein/10 minutes (or 5 minutes where applicable). In preliminary experiments calculations were performed with the aid of a computer. Two programs were used the more complex of which is presented in the appendix, Figure A-I is a flowchart and table A-l is the program listing. The less complicated program was a modification of VIVO to allow for a single value of protein concentration per neurotransmitter (or 
brain region) condition.

Mean values obtained by incubating at $0-4{ }^{\circ} \mathrm{C}$ were routinely subtracted from values obtained at $37^{\circ} \mathrm{C}$ as a control for non-specific adsorption of neurotransmitter to a variety of binding sites including plasma proteins and presumably the transport carrier itself. Uptake was not found to be saturable at $0-4{ }^{\circ} \mathrm{C}$ over the concentration range 0.04-2.0 uM. An untreated sample was used as a value representing maximal uptake with which drug treated samples were compared. Hypothalamic homogenate was used for norepinephrine uptake studies striatal for dopamine and forebrain for serotonin.

1) Determination of neurotransmitter integrity: The radioactive neurotransmitters used in this study were obtained from New England Nuclear Co. as $\mathrm{H}^{3}-1$-norepinephrine ( $\left.\mathrm{H}^{3}-1-\mathrm{NE}: 5.85 \mathrm{Ci} / \mathrm{mM}\right), 5$-hydroxytryptamine binoxalate $\left(\mathrm{H}^{3}-5-\mathrm{HT}: 2.2 \mathrm{ci} / \mathrm{mM}\right.$ ), and dopamine (DA: $1.0 \mathrm{Ci} /$ mM). The corresponding unlabeled compounds were obtained from Sigma Chemical Company as well as the 0-methylated and deaminated metabolites of the neurotransmitters. The unlabeled compounds were spotted on cellulose NM300 thin layer chromatographic plates in $10 \mathrm{ul}$. volumes containing 1-5 ugms. of the test substance as originally described by Giese et al. (1967). The plates were allowed to dry after spotting and were equilibrated in a TIC tank for 30-60 minutes prior to immersion into the solvent (butanol: $5 \mathrm{~N}$ acetic acid=100:35). The solvent travel was spotted after 
approximately $15 \mathrm{~cm}$. Clear separation of all the neurotransmitter was obtained and a test system for neurotransmitter integrity was established by spotting the labeled neurotransmitter. After developing, the visualizable spot was scraped off and placed into a scintillation vial and counted. All adsorbent below the spot was placed into a scintillation vial as was all adsorbent after the spot. High counts found only directly on the visualized spot were interpreted to mean the neurotransmitter remained intact. Once radioactivity either before or after the visualized spot was apparent, the labeled neurotransmitter was no longer used.

ii) Time course for incubation

All samples incubated at $37^{\circ} \mathrm{C}$ were placed in the hot water bath and removed sequentially over the time intervals reported in the results. Another set of tubes identically prepared except that the labeled neurotransmitter was omitted, were placed in an ice-water bath. The labeled neurotransmitter was added at various times during the course of the total incubation time beginning with longest time interval sample (prepared just prior to introducing the $37^{\circ} \mathrm{C}$ samples into the heated water bath). All tubes were vortexed after addition of the labeled neurotransmitter. Results are expressed as Tissue:Medium ratio calculated as ngms $\mathrm{H}^{3}-1-\mathrm{NE} \mathrm{gm}^{-1}$ brain:ngms. $\mathrm{H}^{3}-1-\mathrm{NE} \mathrm{ml} .^{-1}$ supernatant. This experiment was repeated 6 times with the brains of 2-6 animals pooled in different experiments. 
iii) Synaptosomal concentration versus uptake

The synaptosomal concentration was varied by delivering different amounts of the CSH to the IM and adjusting to an identical final sucrose concentration in each tube by the addition of an appropriate volume of $0.32 \mathrm{M}$ sucrose. The tubes were kept in an ice-water bath until the start of the experiment at which time half were transferred to the hot water bath for the previously specified periods of time. The brains of two to six animals were pooled for each experiment and the experiment was repeated five times. The results are expressed as a Tissue (Particulate): Medium ratio calculated ngms. $\mathrm{H}^{3}-\mathrm{I}-\mathrm{NE} / \mathrm{gm}$ brain wet weight: ngms $\mathrm{H}^{3}-\mathrm{I}-\mathrm{NE} / \mathrm{ml}$ and supernatant in an attempt to demonstrate the concentrative property of the synaptosomal preparation.

iv) Effect of calcium ions and ethylenediamine-tetraacetic acid

An attempt to evaluate the contribution of facilitated exchange diffusion in the synaptosomal preparation was made by varying the calcium concentration in the media and employing ethylenediamine tetra-acetic acid (EDTA) to chelate endogenous calcium. Six determinations were made at both 0 and $37^{\circ} \mathrm{C}$. The mean of the six $0^{\circ} \mathrm{C}$ samples was subtracted from each of the $37^{\circ} \mathrm{C}$ values followed by the transformation to uptake expressed as ngms $\mathrm{H}^{3}-\mathrm{l}-\mathrm{NE} / \mathrm{mg}$ ptotein/ 10 minutes. The media of Coyle and Snyder (1969) was used as a control to which greater or lesser concentrations of calcium were compared.

The brains of six aminals were pooled and allquots 
were placed in media that was identical to the media of Snyder and Coyle (1969) except that calcium and EDTA were modified as follows. For the first condition, 1.2mM calcium was replaced with $10 \mathrm{mM} \mathrm{Ca}^{++}$and EDTA was omitted. The calcium concentration was then lowered to $2.5 \mathrm{mM}$ in the second condition. The third condition employed was that of Snyder and Coyle (1969) as a control. In the fourth tube the medium was modified by deleting calcium and adding 0.1 mM EDTA. The EDTA concentrations were increased logarithmically in subsequent tubes to 1.0 and $10 \mathrm{mM}$. The $\mathrm{pH}$ was checked in each and found to require adjustment in the 10 mM EDTA solution by the drop-wise addition of $0.1 \mathrm{~N} \mathrm{NaOH}$ to bring the $\mathrm{pH}$ from 6.6 to 7.4. All other solutions were in the range $7.35-7.45$.

v) Kinetics

Preliminary studies of the kinetics for the transport of biogenic amines into the CSH included all three neurotransmitters, 1-norepinephrine, serotonin and dopamine. Seven substrate concentrations used during preliminary investigations ranged from 2.0 to $0.01 \mathrm{uM}(2-10 \mathrm{uCi} / \mathrm{ml}$.). An aliquot of the supernatant was used as a measure of the total radioactivity in the media since the difference between IM and supernatant counts did not differ by more than $7 \%$ at any time. The brains of 2 animals were pooled and the media of Coyle and Snyder (1969) was used in the studies using serotonin and dopamine as substrate.

l-Norepinephrine kinetics was performed with a calcium- 
free media supplemented with 0.1 uM EDTA. The substrate concentrations ranged from 2.0 to $0.01 \mathrm{uM}(2-10 \mathrm{uCl} / \mathrm{ml}$.). Three determinations were made for each condition and the data was transformed to uptake expressed in ngms $\mathrm{H}^{3}-1-\mathrm{NE} /$ mg protein/10 minutes. The experiment was repeated three times.

vi) Subcellular distribution of $\mathrm{H}^{3}$-l-norepinephrine Continuous sucrose gradients were prepared using a Beckman Density Gradient former resulting in a linear gradient from 0.38 to $1.4 \mathrm{M}$ sucrose in a $12 \mathrm{ml}$. volume. The gradients were prepared 3 hours prior to their use and stored in an ice-water bath until ready for use. The CSH was prepared as an outlined above and after incubation and subsequent centrifugation the pellet was resuspended in a $1.0 \mathrm{ml}$. volume and layered onto the sucrose gradients. An identical aliquot of the CSH was prepared in media in which the monoamine oxidase inhibitor, nialamide, was omitted. Unlabeled l-NE was added to a final concentration of $0.1 \mathrm{UM}$ and processed in an identical manner as the radioactive sample.

The gradients were centrifuged for 90 minutes at $130,000 \mathrm{x} \mathrm{g}$ in an International ultracentrifuge using a swinging bucket rotor. Fractions were obtained by piercing the bottom of polyallomar centrifuge tubes and collecting $0.4 \mathrm{ml}$. fractions.

The samples containing radioactive neurotransmitter was assayed for tritlum by placing $0.1 \mathrm{ml}$. samples into 
scintillation vials containing $2 \mathrm{ml}$. of absolute ethanol to which $10 \mathrm{ml}$. of toluene phosphor was subsequently added.

Unlabeled neurotransmitter samples were assayed for monoamine oxidase activity by a modification of the method of Wurtman and Axelrod (1963). A $0.1 \mathrm{ml}$. aliquot of the sample was added to $0.25 \mathrm{ml}$. of phosphate buffer (pH 7.4) in a test tube. $\mathrm{C}^{14}$-tryptamine was added and the tubes were incubated at $37^{\circ} \mathrm{C}$ for twenty minutes. The reaction was stopped by the addition of $0.2 \mathrm{ml}$ of $2 \mathrm{~N} \mathrm{HCI}$ and the indole acetic acid formed was extracted into $6 \mathrm{ml}$. of toluene by shaking. Four ml. aliquots of the toluene were placed into scintillation vials and $10 \mathrm{ml}$. of toluene phosphor added prior to counting. The results obtained are expressed as percent of radioactivity in each fraction compared with the sum of all fractions.

vii) Regional distribution of neurotransmitter uptake

The uptake of each neurotransmitter into each brain region employed in this study was investigated. The method employed was similar to that outlined above in methods except that all these brain regions were studied for each neurotransmitter. The media used was that described by Snyder and Coyle (1969).

The results are expressed as Particulate:Medium ratio calculated as CPM/mg protein/Incubation time: CPM/ml. supernatant.

viii) Effect of calcium in 1-norepinephrine release induced by hi-potassium depolarization

The role of calcium in the release of norepinephrine 
and its consequent effect on uptake was studied. The hypothalami of ten animals were pooled and divided into equal aliquots. Preincubation media used were as follows: (1-2) hi- $\mathrm{K}^{+}$(45mM) plus $\mathrm{Ca}^{++}(2.5 \mathrm{mM})$, (3) normal Kreb's

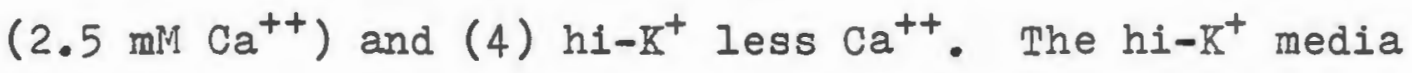
was prepared by equimolar replacement of $\mathrm{Na}^{+}$by $\mathrm{K}^{+}$. The CSH aliquots were incubated in twice the normal incubation medium volume resulting in a protein concentration of 0.24 $\mathrm{mg} / \mathrm{ml}$. Incubation was carried out at $37^{\circ} \mathrm{C}$ for 15 minutes with continuous oxygenation of the media with $\mathrm{O}_{2}: \mathrm{CO}_{2}(95: 5)$. The tubes were then centrifuged at $15,000 \times \mathrm{g}$ for 15 minutes and the resultant pellets reconstituted with modified Kreb's (i.e. no $\mathrm{Ca}^{++}+0.1 \mathrm{mM}$ EDTA) in half the volume used for the preincubation. To tube \#I was added the diluent for the neurotransmitter. To the second tube was added a calculated amount of unlabeled neurotransmitter required to completely refill the synaptosomes (to $1 \mathrm{ugm} / \mathrm{g}$ tissue wet weight). Tube 3 received neurotransmitter diluent as did $\# 4$ and all tubes were incubated for 30 minutes at $37^{\circ} \mathrm{C}$. Following centrifugation at $15,000 \times \mathrm{x}$ for 15 minutes the pellets were resuspended in modified Kreb's media to which $\mathrm{H}^{3}-1-\mathrm{NE}$ was added to achieve a resultant concentration of 0.1 uM and four one $\mathrm{ml}$. aliquots of each tube were incubated at $0^{\circ}$ and eight one ml. aliquots were incubated at $37^{\circ} \mathrm{C}$ for 10 minutes. All tubes were then centrifuged at $13,000 \mathrm{x} \mathrm{g}$ for 60 minutes in a Sorvall RC-2B centrifuge. The pellets were solubilized in the usual manner described in the gen- 
eral methods. The data was calculated as ngm $\mathrm{H}^{3}-\mathrm{I}-\mathrm{N} \mathrm{N} / \mathrm{mg}$ protein/10 minutes and expressed as percent of control.

ix) IC $_{50}$ for some representative tricyclic antidepressants

The ability of tricyclic antidepressants to block the uptake of labeled neurotransmitter by 50 percent (i.e. $I C_{50}$ ) was studied by varying the concentration of antidepressant using 4 concentrations increasing progressively by one order of magnitude. The uptake of neurotransmitter calculated as ngms $\mathrm{H}^{3}-1-\mathrm{NE} / \mathrm{mg}$ protein in the drug treated samples were then compared with an untreated control and expressed as a percent of control. The concentration of drug resulting in uptake representing $50 \%$ of control was determined graphically and described in the results.

$x-x i)$ Protriptyline - acute and subacute time course Protriptyline was administered intraperitoneally either acutely $(20 \mathrm{mg} / \mathrm{kg}$ ) or subacutely $(10 \mathrm{mg} / \mathrm{kg}$ ) at varying times prior to sacrifice as stated in the results. Acute refers to a single injection prior to sacrifice, whereas, subacute refers to 6 consecutive days of injections. On any given day two animals from each treatment condition was represented to randomize day to day experimental variations. The animals were sacrificed at approximately the same time each day necessitatingaschedule of injection running throughout the day (i.e. 5:00, 9:00, 11:00 A.M. and 11:00 P.M.). Three determinations were made for each condition (i.e. $O^{\circ} \mathrm{C}$ and $37^{\circ} \mathrm{C}$ ). Incubations were performed in modified Kreb's 
media ( $\mathrm{nO} \mathrm{Ca}^{++}+0.1 \mathrm{mM}$ EDTA).

xii) Protriptyline - chronic time course

One hundred animals were randomly assigned into five groups of twenty animals each. These five groups were administered either saline or drug beginning on five consecutive days. Within each group of twenty, four groups of five animals each represented either saline injected controls or 2, 6 and 24 hour post-protriptyline ( $10 \mathrm{mg} / \mathrm{kg}$ i.p.) injected treatments. The brains of 5 animals in each group were pooled prior to homogenization and the uptake study was carried out over the course of 5 days after 18 consecutive days of injections for each group of twenty animals. 
C. Results and discussion:

Included in the results sections are discussions pertaining to the individual experiment which serve to develop a sense of continuity and an appreciation for the course of investigation taken in this thesis. The general discussion to follow considers the results of the research presented here as it relates to more general considerations of the possible mechanism(s) of action of tricyclic antidepressants.

The terminology used in the literature with respect to neurotransmitter transport studies is governed largely by the methodology employed (Graefe, 1976). Although the definitions reviewed here are not strictly adhered to in the literature, it is useful to describe the differences and establish the terminology used in this report. "Uptake" of amines can be defined as transmembranous flux into the cell regardless of whether or not intracellular accumulation of the substrate occurs. Many studies attempt to measure "net uptake" which takes into account the accumulation of unchanged amine by the tissue preparation. The tissue preparation can lose accumulated amines by MAO metabolism and a common approach is to inhibit MAO in an attempt to obtain an estimate of net uptake. The use of amines which do not undergo metabolism by MAO (e.g. metaraminol) is an altemate approach to obtain a measure of net uptake. In either case the measurement only approaches the true value of net uptake because exchange processes are 
usually not considered by investigators. There is sufficient evidence to suggest that both hetero-exchange as well as homo-exchange processes do occur (Cohen \& Lajtha, 1972; Levi et al., 1976). Many studies use short incubation times to maximize the magnitude of unidirectional flow of substrate and prolonged incubation usually results in a deviation from linearity. "Re-uptake" refers more to the process in-situ and is inappropriately used as a synonym for uptake. This thesis utilized the term uptake to represent an apparent accumulation of amines and assumes that exchange processes are not significant over the short incubation times used. General:

The reproducibility of the brain dissection procedure is reported in table $I$ and compares favorably with the values reported by Glowinsky and Iversen (1966). Since the boundaries differ some, the difference in values between those published and reported here appear to be reasonable. The weighed brain regions were carried through the homogenization procedure and the functional relationship between the wet weight of brain tissue for a given brain region and the resultant concentration of protein in the IM was determined as shown in figure 1 for the hypothalamus. From the intersection of two regression lines (i.e. $y$ on $x$ and $x$ on $y$ ) the mean values of both variables are obtained. From these values corrections for dilution can be made and the appropriate relationships between $\mathrm{mg}$ protein (as a crude measure of synaptosomal concentration) and a gram of tissue wet weight 
Table 1. Reproducibility of the brain dissection procedure

\begin{tabular}{lc}
\hline Brain region & Tissue wet weight $(\bar{X} \pm$ s.e.m. $)$ \\
\hline Hypothalamus & $133 \pm 8$ \\
Forebrain & $199 \pm 8$ \\
Striatum & $85 \pm 4$ \\
\end{tabular}

Mean weights for various brain regions dissected from 24 animals. 
Figure 1. Functional relationship between brain tissue wet weight and resultant protein concentration in the incubation medium.

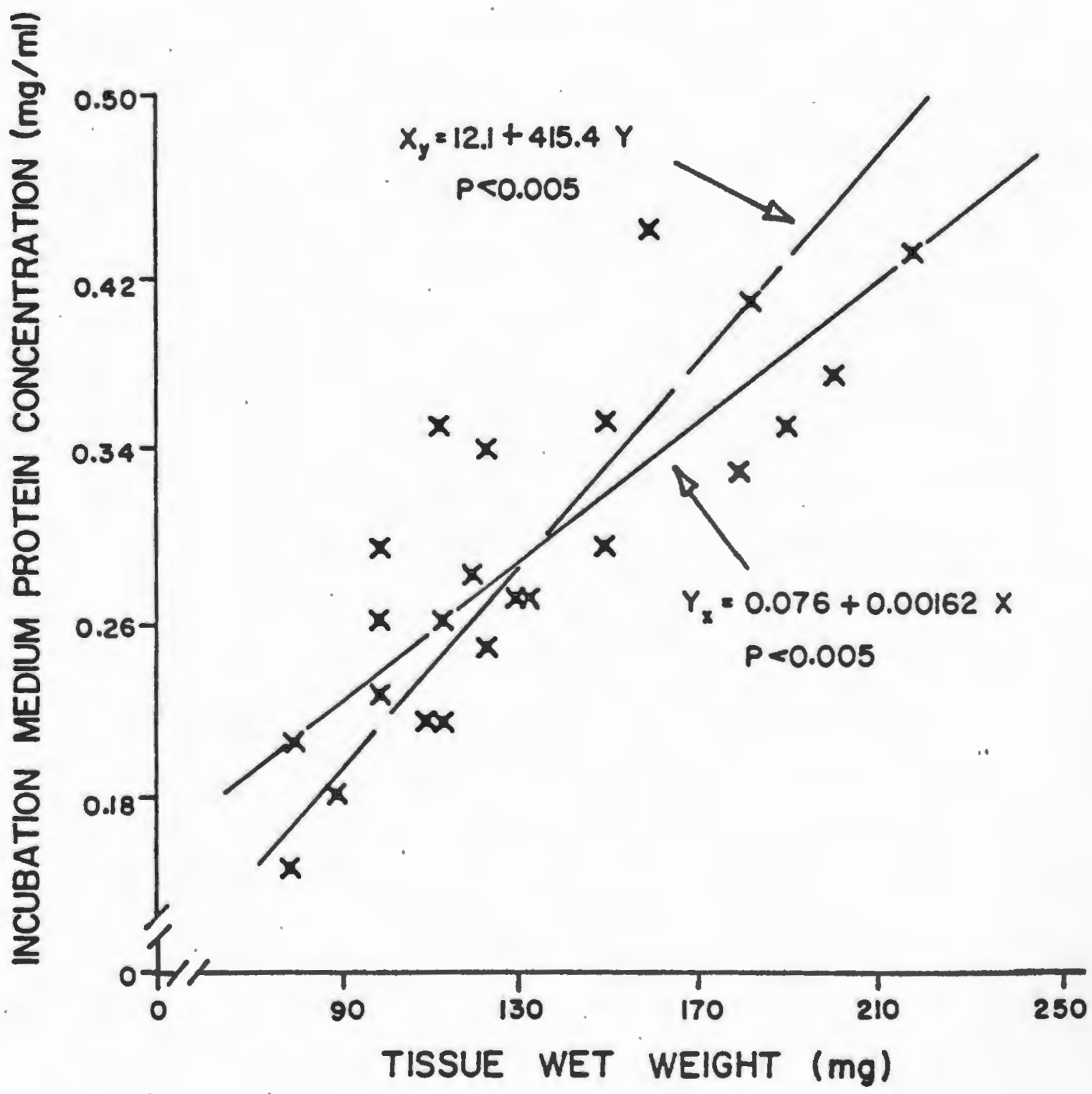


can be formulated. One gram of forebrain, hypothalamus and striatum are equivalent to 460,300 and $285 \mathrm{mg}$ protein respectively. Conversions from mg protein to grams wet tissue weight were made using these relationships.

i) Neurotransmitter integrity

The functional integrity of the substrates used in biochemical systems of the type reported here is an important consideration with respect to interpreting the results. Structual changes of the substrate molecule can result in drastically altering the observed kinetic constants as well as possess inhibitory properties themselves as outlined in the literature review. Monitoring the integrity of the neurotransmitters is important and can be accomplished in at least two ways. A simple method is to use chromatography to identify the substance by separating it from closely related molecular species. The procedure outlined by Giese et al. (1967) and reported here clearly resolved all three neurotransmitters with virtually no overlap in the resultant radio-chromatogram shown in figure 2. De-aminated and 0-methylated metabolites were shown to possess clearly distinguishable spots on the developed chromatogram. The thin layer chromatographic (ILC) procedure was performed between series of experiments on both the unlabeled stock solution as well as the radioactive neurotransmitter.

A method for the detection of specifically inhibitory contaminants in radioactive substrates is described by Timpton et al. (1977). The advantage of this procedure is 
Figure 2. Integrity of radioactive neurotransmitter substrates determined by thin layer chromatography.

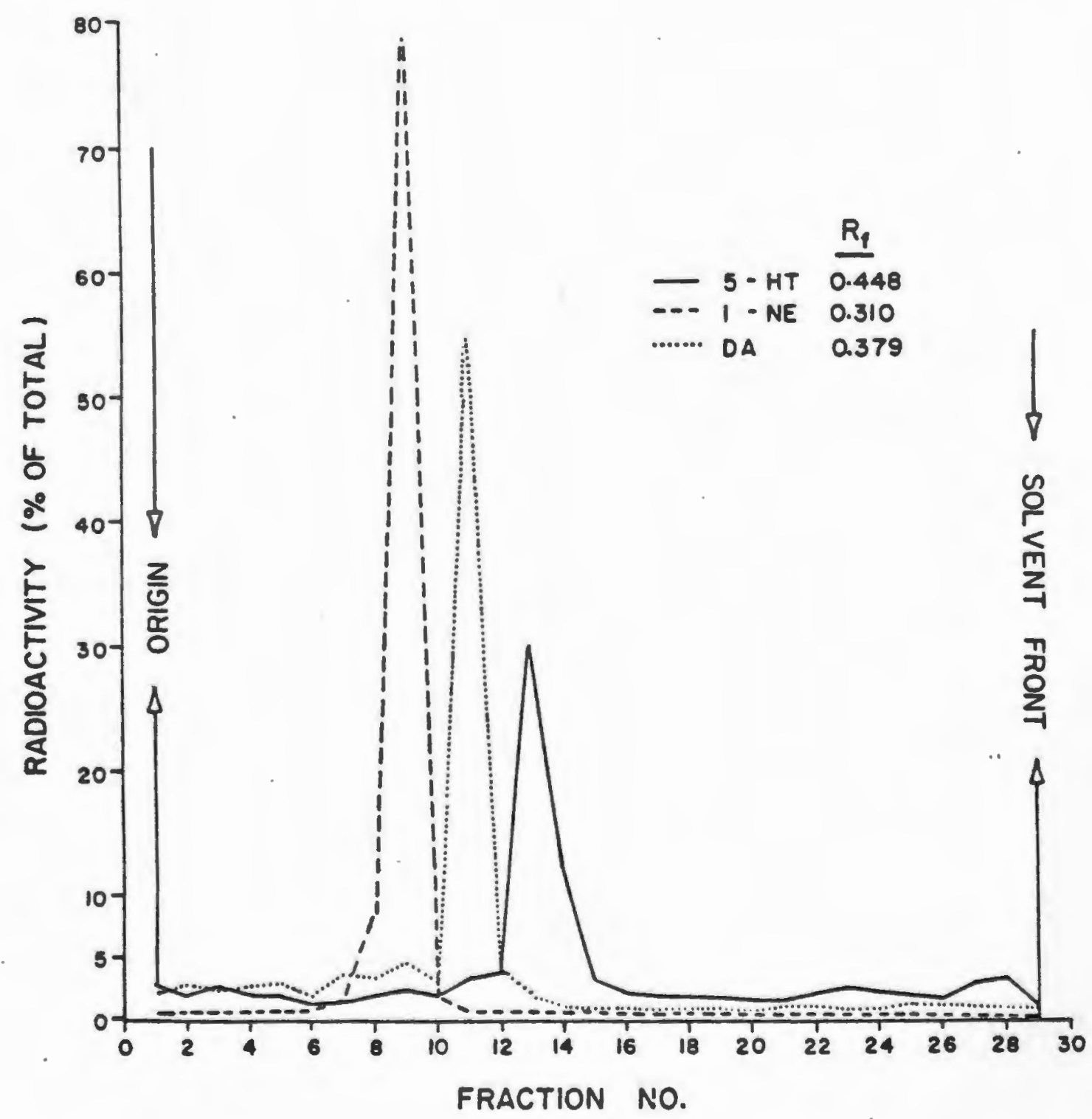


Figure 3. Effect of incubation time upon the uptake of $\mathrm{H}^{3}-I-N E$ into hypothalamic crude synaptosomal homogenate.

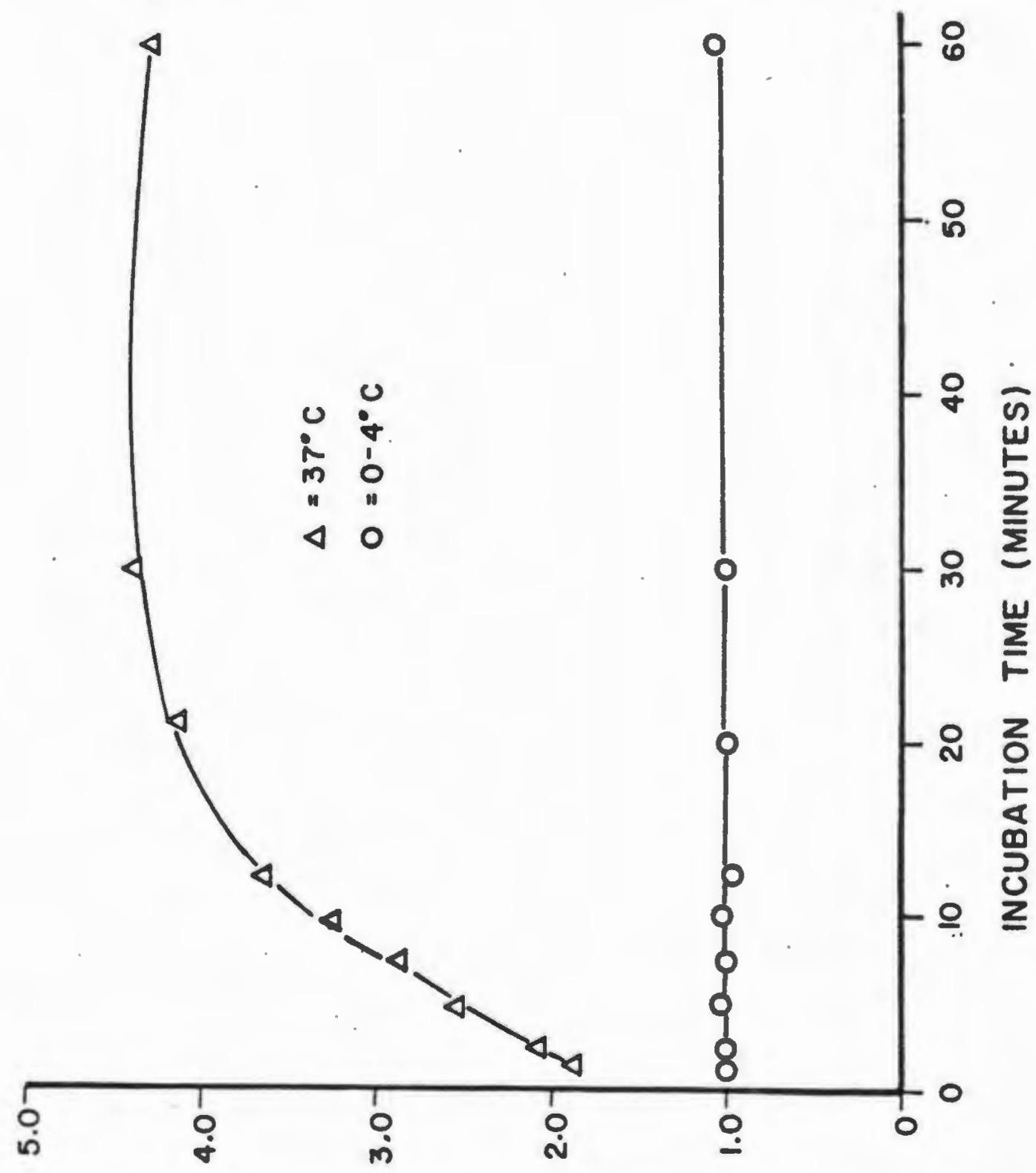

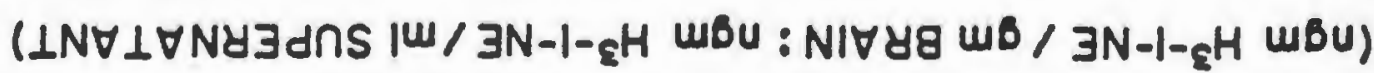

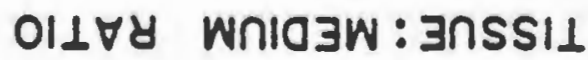


minutes). Similar results were obtained for DA uptake into striatal CSH. These results agree with those published (Coyle and Snyder, 1969; Snyder and Coyle, 1969). Serotonin uptake was linear for six minutes and this value agrees exactly with that reported by Shaskan and Snyder (1970). Wong et al. (1973) demonstrated linearity for 5HT uptake for at least 3 minutes and the difference may be accounted for by the use of a 5 minute pre-incubation or equilibration of the IM prior to introducing the radioactive neurotransmitter and as well as a lower incubation temperature $\left(30^{\circ} \mathrm{C}\right)$. One more complicating factor includes the use of a velocity term as the ordinate of the time course curve presented by these investigators. The velocity term (nM/mg protein/3 min) requires each value for uptake at each time point to be corrected for uptake over 3 minutes. Making the appropriate correction from the velocity term to a concentration term ( $\mathrm{nM} / \mathrm{mg}$ protein) results in a linear function for at least 5 minutes. As a result of these experiments, incubation times for the uptake of $N E$ and $D A$ of 10 minutes were established and 5 minutes for 5-HT. The Tissue:Medium ratio shown in figure 3 demonstrates that the process at $0^{\circ} \mathrm{C}$ seems to be one of adsorption or diffusion whereas at $37^{\circ} \mathrm{C}$ a concentrative phenomenon appears operative with ratios as high as 4 . Dopamine uptake gave ratios one order of magnitude higher than those of 5-HT or NE. The tremendous concentrative power of dopaminergic nerve endings in the striatum may be as a result of finer nerve terminals 
resulting in increased surface area for uptake as suggested by Glowinski and Iversen (1966).

iii) Synaptosomal concentration versus uptake The concentration of tissue in the incubation medium was shown to vary considerably as a result of the variation in the dissection procedure. Another source of this type of variation is the physical size of the brain which varies with the age or weight of the animal. Differences in protein concentration (assumed to be a measure of synaptosomal concentration) was studied in order to understand the nature of this variable and apply whatever corrections seemed necessary. The results shown in figure 4 for $H^{3}-1-N E$ are representative of the results found for the uptake of labeled 5-HT and DA. The points represent the mean of 3 determinations with the coefficient of variation less than $10 \%$ for values above $0.5 \mathrm{mg}$ protein per $\mathrm{ml}$. of IM. Values below $0.5 \mathrm{mg}$ had a much larger degree of variability associated with their means. The resultant curves were calculated using the original data points by polynomial regression. The data was shown to be satisfied best by the second degree polynomial equations shown in the figures with their attendent levels of probability.

Two interesting points can be made by observation of figure 4. First, the ordinate is expressed as uptake in ngms $H^{3}-1-N E$ corrected for mg protein. Theoretically, if increasing synaptosomal concentrations results in an increase in uptake described by a linear function, one might expect 
Figure 4. Fffect of synaptosomal concentration in the incubation medium upon the uptake of $\mathrm{H}^{3}-1-\mathrm{NE}$ into hypothalamic crude synaptosomal homogenate.

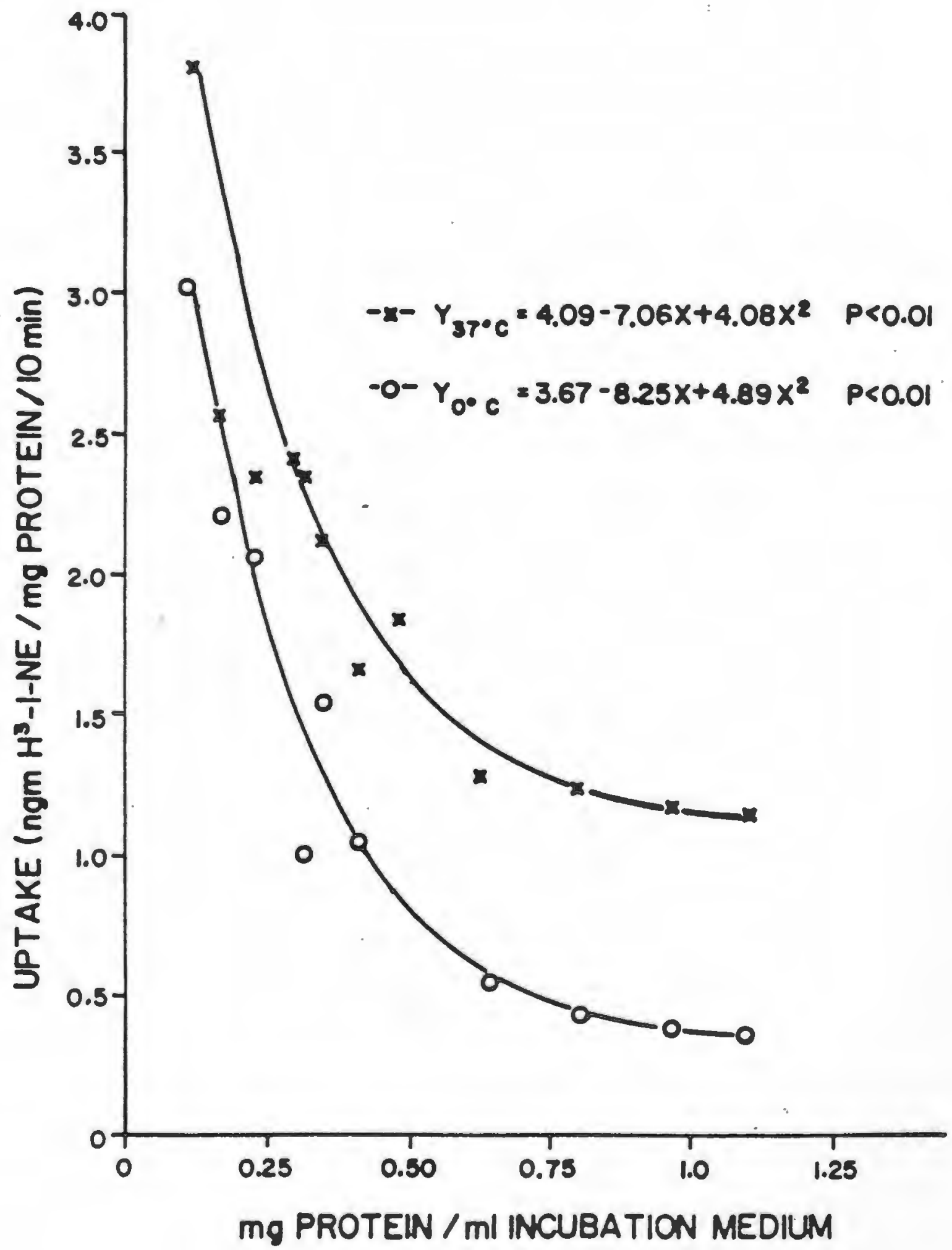


that uptake corrected for $\mathrm{mg}$ protein would be a straight line with a slope close to zero. At concentrations of protein greater than $0.5 \mathrm{mg} / \mathrm{ml}$. this theoretical curve appears to be approximated fairly well.

The tremendous increase in uptake demonstrated at low synaptosomal concentrations may be explained by an increase in membranous surface area. Since the uptake is increased for both 0 and $37^{\circ} \mathrm{C}$ one might conclude that the increase is due to adsorption to the membranous particles whose surface area increases tremendously as a result of dilution. Concentrations above $0.5 \mathrm{mg} / \mathrm{ml}$. may reflect a state where by a certain degree of clumping occurs resulting in minimizing the surface area exposed to media substrate. This clumping effect appears not to increase tremendously over the range $0.5-1.1 \mathrm{mg}$ protein/ml. IM although a slight decline in uptake may be detected with increasing concentrations of protein.

Secondly, it may be noted that the difference between uptake at $37^{\circ} \mathrm{C}$ and $0^{\circ} \mathrm{C}$ is constant for any synaptosomal concentration. This finding is important for it implies there is no effect of packing upon the measure of protein. Interestingly, the relationship holds for low synaptosomal concentrations where, admittedly, the variability is high. An attempt was made to stay within the specified range where variability was lowest. Similar relationships for striatal and forebrain tissue were found and the critical values obtained were 0.16 and $0.25 \mathrm{mg}$ protein per $\mathrm{ml}$. IM respectively. 
iv) The effect of calcium and ethylenediamine-tetra acetic acid

The results of manipulating the calcium concentration in the media are presented in figure 5. A one-way analysis of variance of this data resulted in rejection of the hypothesis that the means were equal at $P<.005$ ( $F_{\text {obs }}=33.9$ compare with $F(5,26 ; \alpha=.005)=4.38)$. An $F_{\max }$ test for homogenity of variance demonstrated no violation of the assumption. A Student-Neumen-Keuls test for differences between treatment means indicated that the Snyder and Coyle (1969) medium resulted in uptake significantly different from that obtained with normal Kreb's medium(Snedecor and Cochran, 1967). When calcium was removed from the mediun and 0.1 m EDTA added, the uptake into hypothalamic synaptosomes was shown to be significantly greater than the value obtained using the medium of Snyder and Coyle (1969). Higher concentration of EDTA resulted in attenuation of the response.

Much mystery still surrounds the role of calcium in stimulus-secretion coupling mechanisms of neurotransmitter release (Rubin, 1970; Phillis, 1974; Baldessarini, 1975). As has been pointed out earlier in this report, preliminary experiments were performed using the mediumdescribed by Snyder and Coyle (1969). The rationale for its use was never made clear in the literature. In 1968, Snyder et al. reported the results of kinetic experiments of racemic $\mathrm{H}^{3}$ $N E$ in brain slices. The mediumdescribed was both normal $\mathrm{Kreb}$ 's and an ascorbate, EDTA and pargyline supplemented 
Figure 5. Effect of altering calcium concentrations in the incubation media upon $\mathrm{H}^{3}-1-\mathrm{NE}$ uptake into hypothalamic crude synaptosomal homogenate.

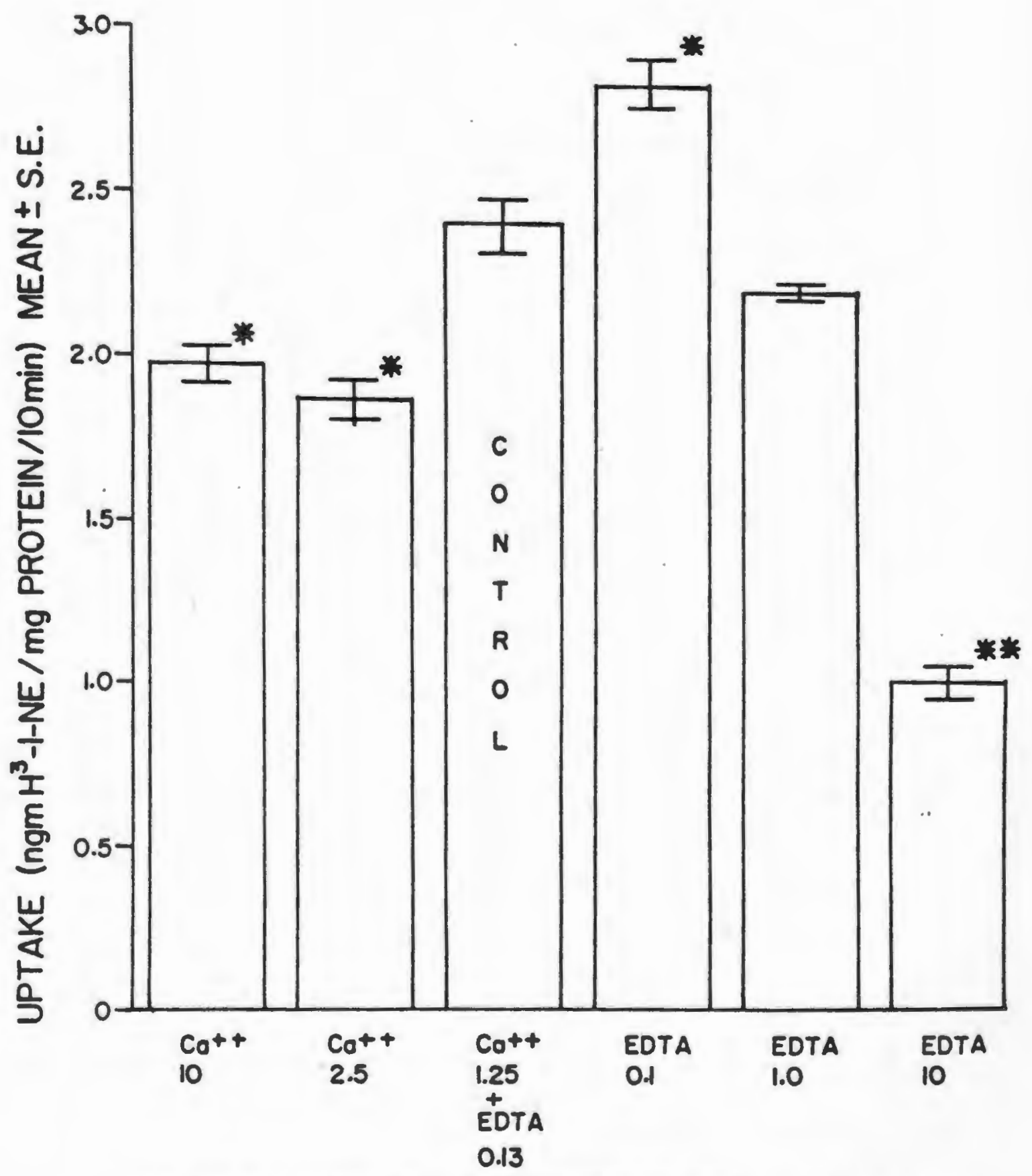

MODIFICATION OF KREB'S INCUBATION MEDIA (mM) 
Kreb's. The authors suggested that IDTA and ascorbate reduced auto-oxidation and pargyline prevented the formation of deaminated metabolic products of $\mathrm{NE}$, but calcium levels were not reduced. Subsequently, Snyder and Coyle (1969) published the use of their calcium reduced medimand it has been used in subsequent studies with no attendant discussion. EDTA is a chelator of divalent cations. The molar ratio of divalent cation to chelate molecule is, at the theoretical limit, equal to two. Since EDTA possesses four carboxylic acid groups it is theoretically possible for two calcium ions to electrically neutralize one EDTA molecule. EDTA, however, is not specific for chelating calcium, Martell and Sillen (1964, 1971) report the metal chelate stabilities of IDTA to be greater for $\mathrm{Cu}$ (11), $\mathrm{Zn}$ (11) and Fe (11) by 70, 49 and $30 \%$ than for calcium. Magnesium has a stability constant $23 \%$ lower than calcium.

Complex ion-chelator formations may also occur in acidic media by protonation of the amine groups. Therefore, it is with considerable conserative bias that the theoretical stoichiometric limit of 2 moles of $\mathrm{Ca}^{++}$required to form a complex with one mole of EDTA is used to calculate divalent ion equivalency in the various media employed. Theoretically then, $0.13 \mathrm{mM}$ EDTA would complex $0.26 \mathrm{mP} \mathrm{Ca}^{++}$rendering the media of Snyder and Coyle equivalent to $0.99 \mathrm{mM} \mathrm{Ca}^{++} \mathrm{Kreb}^{\mathrm{s}}$. In consideration of the conservativeness eluded to above, a $\mathrm{Ca}^{++}$concentration slightly higher than this might be more equivalent to the Snyder and Coyle medium. 
In a separate experiment the uptake of 1-NE into hypothalamic CSH was measured using the Snyder and Coyle medium compared with $1.25 \mathrm{mM} \mathrm{Ca}^{++} \mathrm{Kreb}$ 's. The results, analyzed using a two-tailed t-test did not show the means to be significant at $P<.05$ although the $1.25 \mathrm{mM} \mathrm{Ca}^{++}$mean value obtained was approximately $9 \%$ below the value obtained using the Snyder and Coyle medium. Acceptance of the null. hypothesis suggests that the two media may be equivalent with respect to the availability of $\mathrm{Ca}^{++}$ions.

The media of snyder and coyle was chosen in an attempt to duplicate the experimental protocol and compare the results as an aid to characterization of the system. Subsequent to these preliminary studies use of the medivir was questioned and prompted this experiment. The choice of deleting calcium and adding EDTA was made using purely theoretical considerations. Since uptake studies attempted measuring unidirectional flux it was reasoned that efflux of endogenous amines in calcium enriched media might confound the results. Indeed both homo-and hetero-exchange processes are known to occur in transport systems of neurotransmitter amino acids (Cohen and Lajtha, 1972). , Preventing the calcium dependent process would serve to reduce the accumulation of label by reducing its specific activity in the IM. Controlling dilution by calcium-independent or spontaneous release of endogenous neurotransmitter is not possible and should be considered as a source of error in any estimate of unidirectional transmembranous flux of 
neurotransmitter molecules.

The results indicate that removal of calcium, up to a point, does result in an increase in the value of uptake. Increasing the EDTA concentration beyond $0.1 \mathrm{mM}$ results in a dose dependent decline in uptake. Interestingly, Rajan et al. (1977) reported a dose dependent inhibition of $\mathrm{H}^{3}-\mathrm{NE}$ binding by metal chelators in mouse cerebral vesicles in vitro. It is possible then that high concentrations of chelating agent interfere with metal coordination complex formation within vesicles. It is also possible that since the chelating agents bind to copper and iron, high concentrations of chelators may interfere with cell respiratory processes requiring these metal ions. Either mechanism may result in the attenuation of uptake demonstrated at high EDTA concentrations.

Blaustein et al. (1972) demonstrated a 37\% decrease in synaptosomal norepinephrine release from a calcium-free normal Kreb's medium compared with $2.5 \mathrm{mM} \mathrm{Ca}^{++}$normal Kreb's. The magnitude of this release, calculated from the data provided in the paper and making the assumption that one rat brain weighs approximately 2 grams, results in a value of $2.2 \mathrm{ng} \mathrm{NE} / \mathrm{g}$ wet weight/min released into the normal $\mathrm{Kreb}$ 's medium and $1.45 \mathrm{ng} \mathrm{NE} / \mathrm{g}$ wet weight/min in $\mathrm{Ca}^{++}$-free Kreb's. The data presented in figure 5 demonstrates a $54 \%$ increase in uptake using a $\mathrm{Ca}^{++}$-free EDTA-enriched medium when compared with normal Kreb's. The value of uptake obtained in the experiments described in this thesis are 
5.5 and $8.5 \mathrm{ng} / \mathrm{NE} / \mathrm{g} / \mathrm{min}$ in normal $\mathrm{Kreb}$ 's and calcium free EDTA enriched Kreb's respectively. These figures indicate that $\mathrm{Ca}^{++}$independent or spontaneous neurotransmitter release may contribute significantly to homoexchange processes confounding a measurement of unidirectional flux into synaptosomes.

Kant and Meyerhoff (1977) studied the effect of calcium on norepinephrine release from hypothalamic tissue minces. These authors compared the release of $\mathrm{NE}$ in a normal $\mathrm{Kreb}$ 's versus a calcium free Kreb's media at $0^{\circ} \mathrm{C}$ and reported a seemingly insignificant increase (5\%) of release in normal Kreb's. Iane and Aprison (1977) reported all three neurotransmitters (i.e. NE, DA \& 5-HT) to behave similarly. Since the release of endogenous neurotransmitter can be minimized by modifying the media all subsequent experiments reported here have been performed with calcium-free EDTA enriched ( $0.1 \mathrm{mM}$ ) Kreb's media referred to herein as "modified Kreb's".

v) Kinetics

Characteristics of transport systems of the type used in this study have been determined using relationships described by enzyme kinetics. Iinear transformations of the Michaelis-Menten equation leads to graphical techniques for the determination of kinetics constants (i.e. $\mathrm{Km}$ and $V_{\max }$ ). A number of linear transformations are possible and most are reviewed in standard texts on enzyme kinetics (Roberts, 1977). In recent years a growing number of investigators have become concerned with the validity of the methods employed. 
The most popular method by far is that of Lineweaver-Burk (1934), although Dowd and Riggs (1965) demonstrated that this method led to least accurate determinations of kinetic constants whether the curve was plotted by eye or using the method of least squares. The Hofstee plot (S/v vs. S) was considered the most favorable since it was viewed that of the two variables $S$ had less error associated with it than v. Estimates of kinetics constants can be obtained using non-linear statistical techniques (Cleland, 1967; Wentworth, $1965 \mathrm{a}, \mathrm{b})$ but these methods require 1terative procedures and have not demonstrated popularity among biochemists. Elmore et al. (1963) applied a linear regression technique to the Lineweaver-Burk transformation which accounted for the varlability associated with each data point. Wilkinson (1961) applied a similar technique except that a weighting factor was used with the assumption that the distribution of the observations of the velocity about a given substrate concentration was directly proportional to the magnitude of the velocity. This assumption can be shown to be true in many biological systems where analysis using MichaelisMenten kinetics apply. Probably the best method from the standpoint of a mathematical approach to the determination of kinetic constants is that of Wilkinson (1961) although it requires the assumption of normally distributed data as any parametric method does.

Recently, a graphical method for the analysis of kinetic data called the direct linear plot (DLP) has been 
reported by Eisenthal and Comish-Bowden (1974). Non-para metric statistical considerations supporting the use of this method have been described (Comish-Bowden and Eisenthal, 1974; Trager and Porter, 1977). The advantage to the direct linear plot: over the other methods as pointed out by the authors is that it is simple to construct with kinetic constants read directly off the graph without calculations as is done with reciprocal plots. It is extremely sensitive to outliers or abberant observations and provides accurate determinations of kinetics constants.

The kinetics data for 1-NE uptake into hypothalamic CSH was analyzed using a number of different methods, some graphical and some statistical. The results obtained by various methods are shown in table 2. The easiest to prepare. The kinetics constants obtained were compared and the results obtained indicate the direct linear plot to be as good an estimator of the kinetics constants obtained by either Wilkinson (1961) regression or the standard parametric linear regression model described by Elmore et al. (1963).

The DIP was used in the calculation of all kinetics data reported here and is described briefly to acquaint the reader with the technique. The interester reader is refered to the original references of Cornish-Bowden and Eisenthal for the theory and a more thorough discussion of techniques and applications. The DIP is so named "...because it represents each observation by a straight line, ..." and utilizes the concept of plotting data in parameter space rather than 
Table 2. Comparision of methods used to determine kinetics constants for $\mathrm{H}^{3}-\mathrm{l}-\mathrm{NE}$ uptake into rat hypothalamic CSH

\begin{tabular}{|c|c|c|}
\hline $\mathrm{Km}(\mathrm{uM})$ & $\underline{\mathrm{v}}_{\mathrm{max}}(\mathrm{ng} / \mathrm{mg} \operatorname{prot} / 10 \mathrm{~min})$ & Method \\
\hline 0.308 & 2.85 & $1 / \mathrm{v}$ vs. $1 / \mathrm{s}$ \\
\hline 0.239 & 2.434 & $S / v$ vs. $S$ \\
\hline 0.236 & 2.40 & v/s vs. v \\
\hline $\begin{array}{l}0.262 \\
\pm .018\end{array}$ & $\begin{array}{l}2.539 \\
\pm .098\end{array}$ & $\begin{array}{l}\text { reduced weighted } \\
\text { regression } \\
\text { assuming constant } \\
\text { coefficient of } \\
\text { variation }\end{array}$ \\
\hline 0.276 & -2.628 & $\begin{array}{l}\text { weighted linear } \\
\text { regression }\end{array}$ \\
\hline 0.235 & 2.340 & $\begin{array}{l}\text { Direct Iinear } \\
\text { Plot }\end{array}$ \\
\hline
\end{tabular}

\author{
$\underline{\text { Reference }}$ \\ Lineweaver-Burk, 1934 \\ Woolf plot \\ (Haldane \& Stern, 1932) \\ Augustinson (Hofstee) \\ Johansen \& Lumry, 1961; \\ Wilkinson, 1961 \\ Steel and Torrie, 1960; \\ Elmore, Kingston \& \\ Shields, 1963 \\ Eisenthal \& Cornish- \\ Bowden, 1974
}


observation space. The ordinate is labeled $V_{\max }$ and the abscissa $\mathrm{Km}$ and the corresponding set of observations, 1.e. $\checkmark$ and $S$, are represented as points on the abscissa and ordinate, respectively. A line is drawn through each set of data points and is continued as far as possible into the first quandrant. The data for 1-NE kinetics is show in figure 6 using the DIP. It can be demonstrated that for $n$ pairs of observations there exists $1 / 2 n(n-1)$ intersections of the lines extended into the first quandrant. The nonparametric theory specified the most likely unbiased estimator of the parameters to be determined is given by the point associated with the median of all interecting lines. That point can be taken from the associated values of the axes labeled $\mathrm{Km}$ and $\mathrm{V}_{\max }$ * For 6 data points there are $6 / 2$ $(6-1)=15$ intersections with the median being the point described between the points designating the seventh and eighth intersecting lines. Two intersecting lines yields one point whereas three intersecting lines coming close to the same place can be shown to be represented by three points. Counting may begin from the low left corner of quadrant 1 and proceed to the upper right. The first circle represents 4 intersecting lines or 5 intersections. Since we are interested in that intersection which falls between 7 and 8 , that intersection must be in the second circle (i. e. between intersections 4 and 9).

Comparisons of the kinetic data obtained using the DIP with reported values are presented in tables 3, 4 and 5 for 
Figure 6. 'Direct Iinear Plot' of $\mathrm{H}^{3}-\mathrm{I}-\mathrm{NE}$ uptake into hypothalamic crude synaptosomal homogenate.

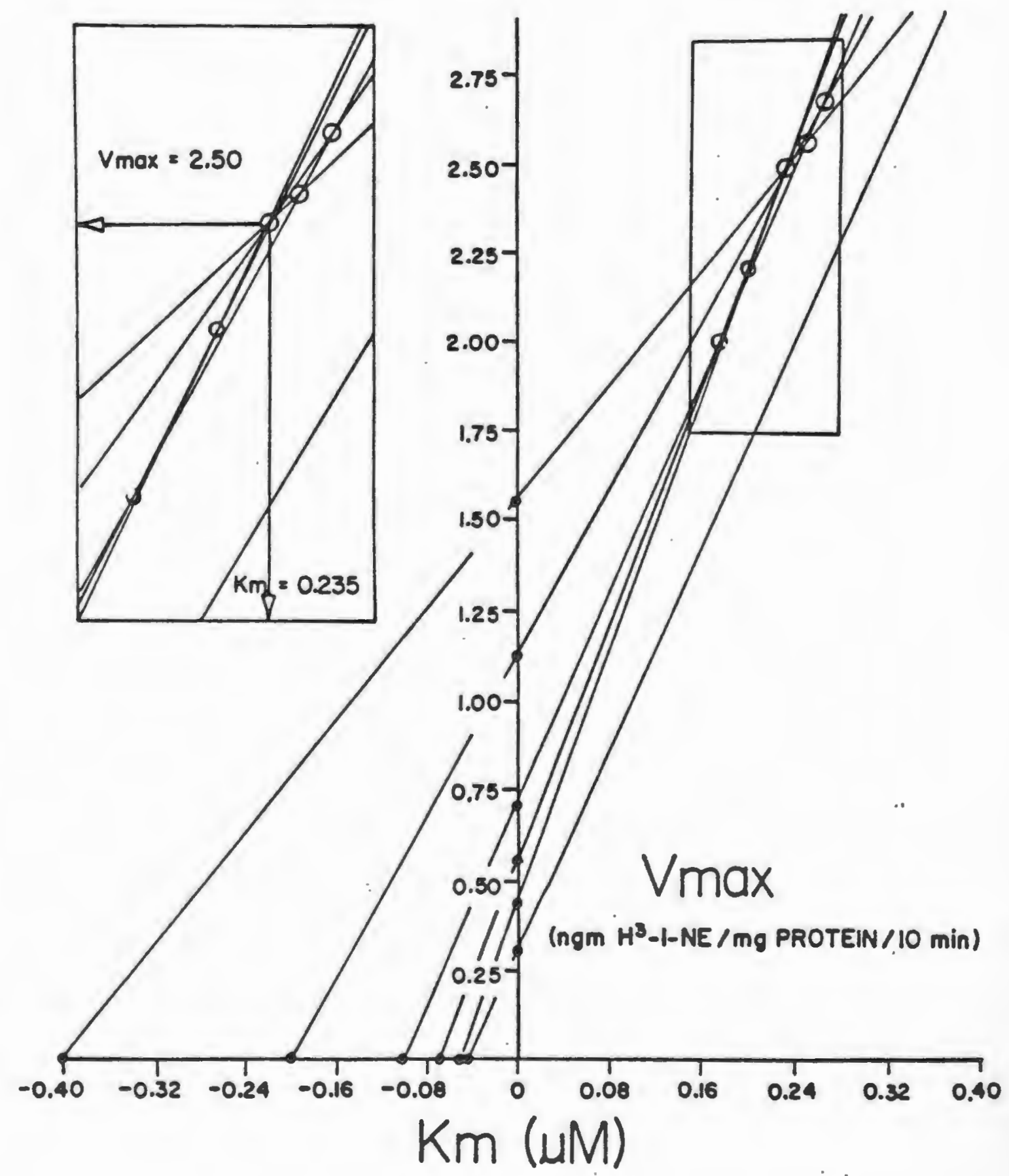


Table 3. Kinetic constants obtained for norepinephrine uptake using substrate concentrations in the high affinity range.

\begin{tabular}{|c|c|c|c|}
\hline $\begin{array}{l}\mathrm{Km} \\
(u M)\end{array}$ & $\left(\mathrm{nM} / \mathrm{g}^{\mathrm{max}} \mathrm{x}_{\min }\right)$ & Reference & Remarks \\
\hline .0 .4 & 0.11 & Ross and Renyi, 1964 & $\begin{array}{l}\mathrm{d}, \mathrm{l}-\mathrm{NE} \text { mouse } \\
\text { cortex slices }\end{array}$ \\
\hline .0 .48 & 0.71 & Salama et al., 1971 & $\begin{array}{l}\text { d, I-NE rat } \\
\text { brain slices }\end{array}$ \\
\hline 0.4 & 1.66 & Snyder et al., 1968 & $\begin{array}{l}\mathrm{d}, \mathrm{l}-\mathrm{NE} \text { rat } \\
\text { brain slices }\end{array}$ \\
\hline 0.2 & $\mathrm{~N} / \mathrm{A}$ & Ross and Renyi, 1975 & $\begin{array}{l}\text { I-NE rat brain } \\
\text { synaptosomes }\end{array}$ \\
\hline 0.5 & $\mathrm{IN} / \mathrm{A}$ & Ross and Renyi, 1975 & $\begin{array}{l}\text { l-NE rat brain } \\
\text { slices }\end{array}$ \\
\hline $0.2-0.4$ & $0.33-0.40$ & Shaskan and Snyder, 1970 & $\begin{array}{l}\mathrm{d}, \quad 1-\mathrm{NE} \\
\text { synaptosomes } \\
\text { hypothalamus }\end{array}$ \\
\hline 0.227 & 0.413 & This study & $\begin{array}{l}\text { calcium-free plus } \\
\text { EDTA enriched } \\
\text { medium }\end{array}$ \\
\hline
\end{tabular}


Table 4. Kinetic contants obtained for serotonin uptake using substrate concentrations in the high affinity range.

$\begin{array}{llll}\mathrm{Km} & \mathrm{V}_{\text {max }} & \text { Reference } & \text { Remarks }\end{array}$

\begin{tabular}{|c|c|c|c|}
\hline 0.7 & 0.23 & Ross and Reny1, 1969 & $\begin{array}{l}\text { mid-brain } \\
\text { mouse slices }\end{array}$ \\
\hline 0.1 & 1.13 & Wong et al., 1973 & $\begin{array}{l}\text { rat brain } \\
\text { synaptosomes }\end{array}$ \\
\hline $0.14-0.31$ & $0.66-0.82$ & Shaskan and Snyder, 1970 & $\begin{array}{l}\text { rat brain } \\
\text { synaptosomes }\end{array}$ \\
\hline 0.08 & $\mathrm{~N} / \mathrm{A}$ & Ross and Renyi, 1975 & $\begin{array}{l}\text { rat brain } \\
\text { synaptosomes }\end{array}$ \\
\hline 0.6 & $\mathrm{~N} / \mathrm{A}$ & Ross and Renyi, 1975 & $\begin{array}{l}\text { rat brain } \\
\text { slices }\end{array}$ \\
\hline 0.15 & 0.84 & This study & $\begin{array}{l}\text { Medium of } \\
\text { Snyder and } \\
\text { Coyle (1969) }\end{array}$ \\
\hline & & & \\
\hline
\end{tabular}


Table 5. Kinetic constants obtained for dopamine uptake using substrate concentrations in the high affinity range.

$\underset{(\mathrm{Km})}{\mathrm{Km}} \quad\left(\mathrm{nM} / \mathrm{g}^{\mathrm{V}^{\mathrm{max}}} \mathrm{m}_{\mathrm{m}}\right) \quad$ Reference

$0.31-0.4 \quad 4.08-5.0 \quad$ Shaskan and Snyder, 1970

$0.13 \quad 5.08 \quad$ This study 
$\mathrm{NE}, 5-H T$ and DA respectively. The data appears in good agreement with the values reported by other investigators. Small differences may be due to the tissue preparation used (slices vs. synaptosomes) as well as the duration of incubation which can result in larger differences in the $\nabla_{\max }$ values. These results suggest that the experimental protocol as used here yields results that are similar to those obtained in other laboratories. Further support for this conclusion is offered in a review of the in-vitro data of section ix.

vi) Subcellular distribution of $\mathrm{H}^{3}$-norepinephrine uptake

An attempt to characterize the functional integrity of the synaptosomal transport system was made by separating some of the constituents of the CSH. The synaptosomes are known to be confined to a region on the sucrose gradient corresponding to 1.1 molar (Gray and Whittaker, 1962). Mitochondria are known to sediment below $1.2 \mathrm{M}$ sucrose and monoamine oxidase activity is taken to be a marker for mitochondria (Laduron, 1977). In figure 7 it can be observed that the radioactive neurotransmitter concentration is highest at the synaptosomal fraction and some label can be seen associated with myelin and microsomes as might be expected due to adsorption. The supernatant possesses a large degree of label but little or no MAO activity. This evidence suggests that the CSH is selectively incorporating radioactive neurotransmitter within the synaptosomal fraction and there is little contamination of uptake into either 
F1gure 7. Subcellular distribution of previously accumulated H3-I-NE into hypothalamic crude eynaptosomal homogenete.

\section{SUCROSE (M)}
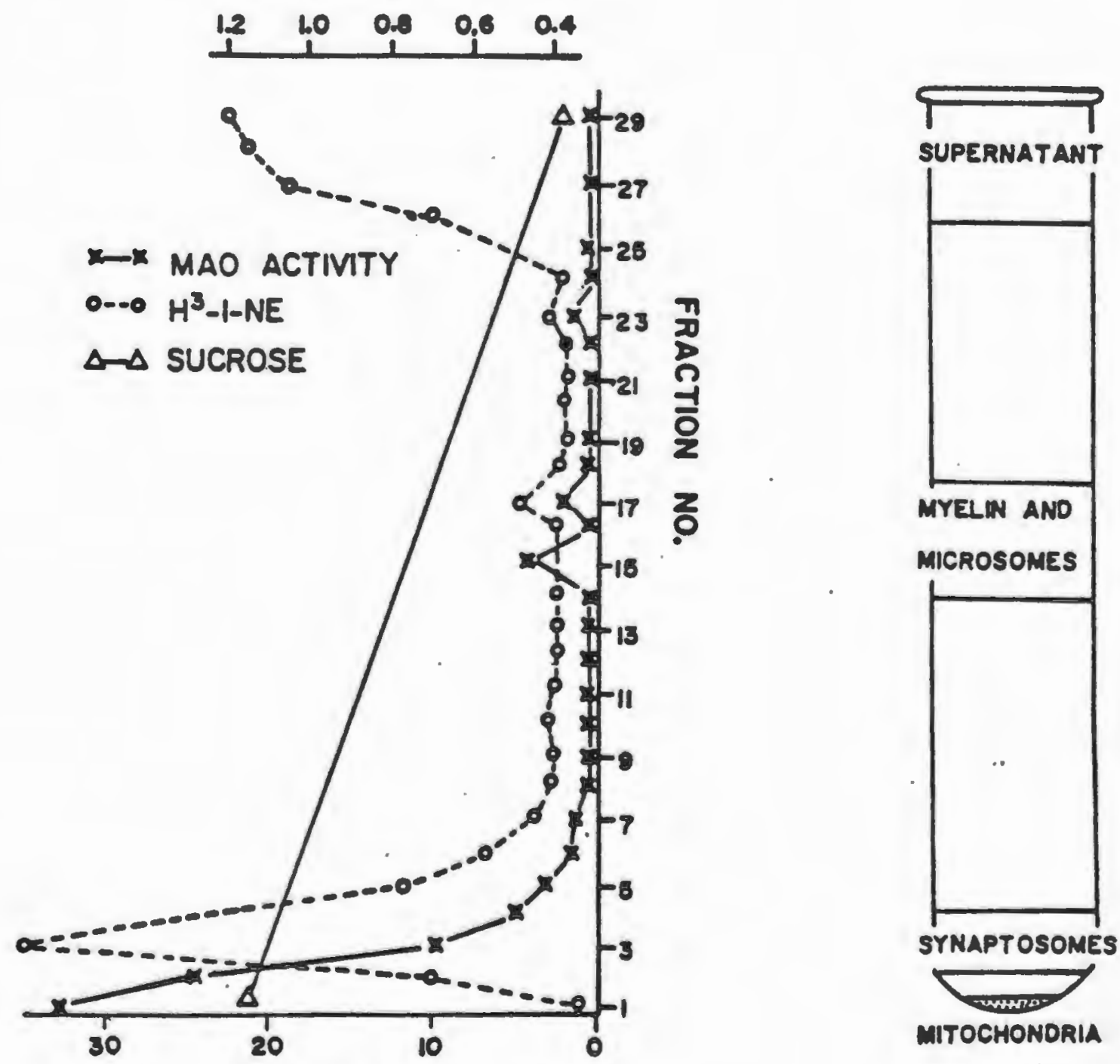

\% TRITIUM AND MAO ACTIVITY 
mitochondrial or microsomal components.

vii) Regional distribution of neurotransmitter uptake

The selection of brain regions for studying the uptake of different neurotransmitters was based on the rationale that selection of brain regions with a high concentration of nerve terminals would result in a greater degree of uptake compared with non-specific binding. Snyder and coyle (1969) used hypothalamus for norpeinephrine and striatum for dopamine uptake studies. Kuhar et al. (1972) used forebrain for serotonin uptake studied. Confirmation of this selection of brain regions was attempted by studying the uptake of each neurotransmitter in each brain region. Figure 8 depicts the results. Each value and its associated variation represents the results obtained from 3 animals. Dopamine uptake is unquestionably greatest into the striatal tissue although a fair amount of dopamine can be taken up into hypothalamic tissue presumably into noradrenergic as well as dopaminergic neurons to some degree (Coyle \& Snyder, 1969). Norepin- . phrine uptake, although large in the striatum, may represent uptake into dopaminergic and other nerve terminals. Hokefelt (1970) estimated the noradrenergic contribution towards total catecholamines in the striatum to be $5 \%$ based on histochemical observations. The selection of tissue for uptake is similarly clear with greatest values obtained for serotonin uptake into forebrain tissue.

The choice of $N E$ into hypothalamus appears to be governed more by tradition and concentrations of all three 
Figure 8. Regional distribution of high affinity neurotransmitter uptake.

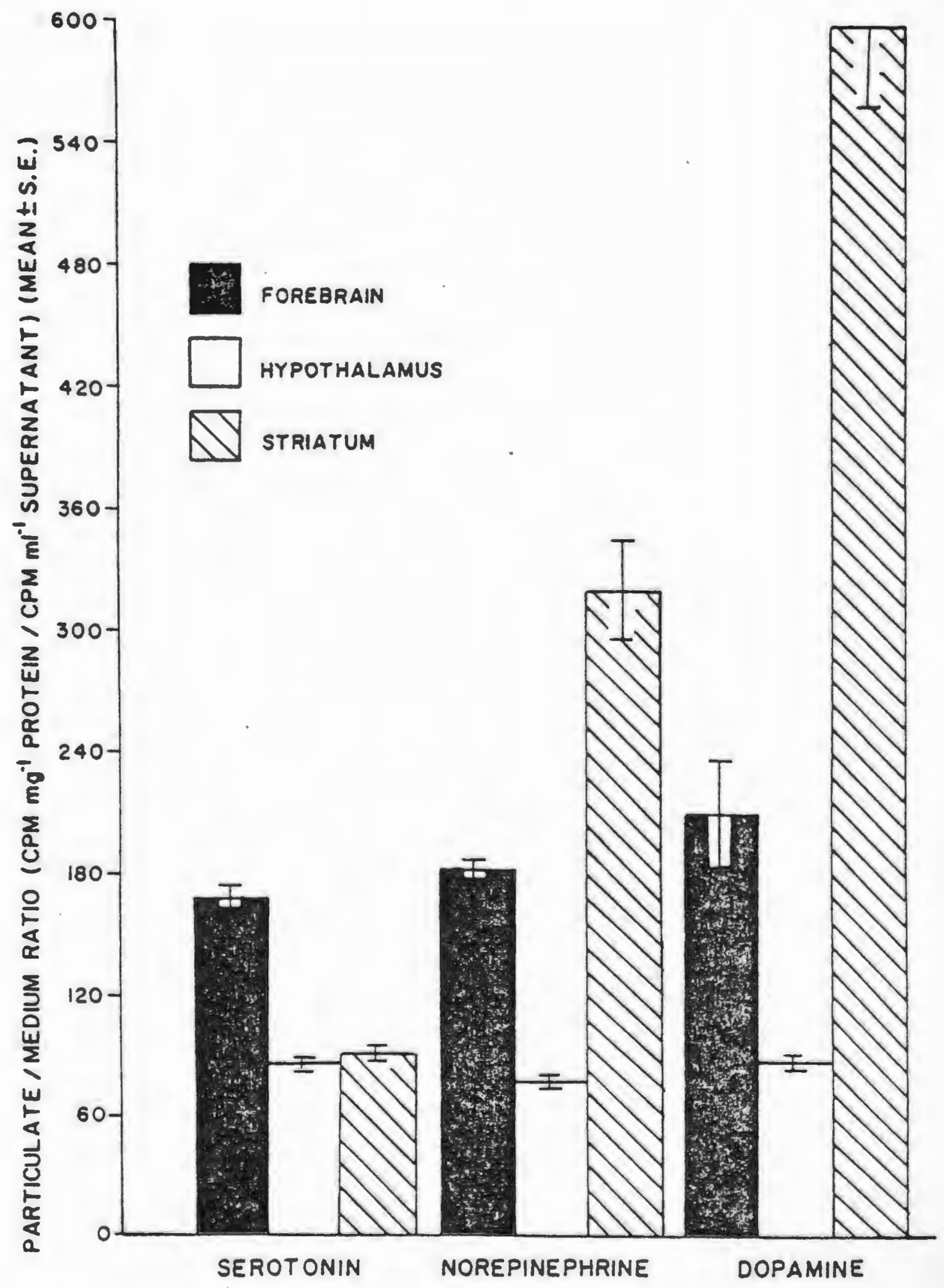


84

neurotransmitters are shown to be equivalent in the hypothalamus (Anden et al., 1966).

viii) Effect of calcium in 1-norepinephrine Induced release by hi-potassium depolarization

This study was prompted by the observation that a tremendous degree of variability occured in the in-vitro measurement of uptake after intraperitoneal administration of protriptyline. In an attempt to uncover the source of this variability it was reasoned that endogenous neurotransmitter may influence uptake. The results of this experiment are presented in table 6. Although the data presented here could not be shown to differ using an analysis of variance, the data can be taken to. suggest a trend. Stimulation of release of endogenous neurotransmitter by $h i-\mathrm{K}^{+}$depolarization resulted in greater uptake when compared with tissue identically processed in normal Kreb's. Depleting the synaptosomes by $\mathrm{hi}^{+} \mathrm{K}^{+}$in the absence of $\mathrm{Ca}^{++}$results in a value of uptake intermediate between what can be considered a greater and lesser degree of emptying. Refilling the synaptosomes with unlabeled NE and subsequently incubating with labeled neurotransmitter results in a value higher than that obtained for the same treatment except for refilling.

The results appear predictable. Removing neurotransmitter from the synaptosomes may reasonably result in greater uptake by increasing the physical capacity to accumulate neurotransmitter taken up. Kant and Meyerhoff (1977) demonstrated at $17.5 \%$ increase in NE released by 
Table 6. Effects of Hi- $\mathrm{K}^{+}$depolanization pretreatment upon subsequently measured $\mathrm{H}^{2}-1-\mathrm{N}$ I uptake

Tissue:Medium ratio (CPM/gm. brain:CPM/ml. supernatant) Percent of Control (Means \pm s.e.m.)

\begin{tabular}{|c|c|c|c|}
\hline Normal Kreb's & $\underset{\text { with } \mathrm{Ca}^{++}}{\mathrm{Hi}-\mathrm{K}^{+}}$ & $\begin{array}{c}\mathrm{Hi}-\mathrm{K}^{+} \\
\text {without } \mathrm{Ca}^{++}\end{array}$ & $\begin{array}{l}\mathrm{ND} \text { prefilling } \\
\text { Ei-K } \\
\text { with } \mathrm{Ca}^{++}\end{array}$ \\
\hline $100 \pm 27$ & $160 \pm 43$ & $139 \pm 18$ & $177 \pm 49$ \\
\hline
\end{tabular}


$\mathrm{Hi}-\mathrm{K}^{+}$in the absence of $\mathrm{Ca}^{++}$which can be used to explain the reason for an increase in uptake over normal Kreb's. The fact that uptake is increased in normal $\mathrm{Ca}^{++} \mathrm{Hi}-\mathrm{K}^{+}$as opposed to $\mathrm{Ca}^{++}$free $\mathrm{Hi}-\mathrm{K}^{+}$media suggests that exogenously administered NE may equilibrate with both neurotransmitter compartments (i.e. vesicular and cytoplasmic).

The increase in uptake measured in the refilling condition appear inconsistent with notions regarding the distribution of neurotransmitter within the synaptosome. Classical theory considers norepinephrine to be bound with a protein, chromagranin $\mathrm{A}, \mathrm{ATP}$ and $\mathrm{Mg}^{++}$(Cooper, Bloom \& Roth, 1974). It is also postulated that a mobile pool exists within the vesicle (Kirshner, 1962). The exact relationship between the uptake of exogenously administered norepinephrine and subsequent compartmentalization between vesicles and cytoplasm is not known. Release studies using preloading techniques make the assumption of equal distribution inherently. It might appear then that previously accumulated norepinephrine distributes itself to some extent in both compartments and in doing so may result in inhibiting any further uptake of exogenously applied neurotransmitter by mass action. The results indicate quite the contrary and may be interpreted to reflect an uneven distribution within compartments. Although admittedly speculative, one explanation may be that recently accumulated neurotransmitter is inactivated or bound by vesicular complexing components. This binding would reduce the outward 
flow of previously accumulated neurotransmitter and may result in a priming effect on intra-vesicular Mg-ATP-protein complex. Alternatively, high cytoplasmic concentrations of accumulated $N E$ may result in a greater rate of exchange so that uptake may appear to increase overall but net uptake does not change. Answers to these questions are experimentally approachable and in view of the fact that Grahame-Smith and Parfitt (1970) report similar results with 1-tryptophan, a study of this type may help to elucidate the compartmentalization of exogenously administered neurotransmitter.

ix) IC $_{50}$ for some representative tricyclic antiThere are, as in obtaining kinetics constants, different methods used to calculate the $I_{50}$ for a compound with respect to particular response. Ross and Renyi (1967) introduced the concept of an uptake blocker standard $(1.0 \mathrm{x}$ $10^{-4} \mathrm{M}$ cocaine) by which other compounds of varying doses could be compared. The data is reported as percent of total inhibition and the $I C_{50}$ calculated by graphic observation of the resultant log dose-response curve. Another alternative is to use a vehicle-treated (or untreated) control and compare the value obtained for drug treated samples with those obtained for controls and represent the data graphically as in the log dose-response curve of figure 9. The results in this figure are the means obtained in four separate experiments when drug treated samples wherecompared with an untreated control. The resultant points were connected by a straight line using visual methods and the point at which 
Figure 9. Graphlcal determination of IC 50 's.

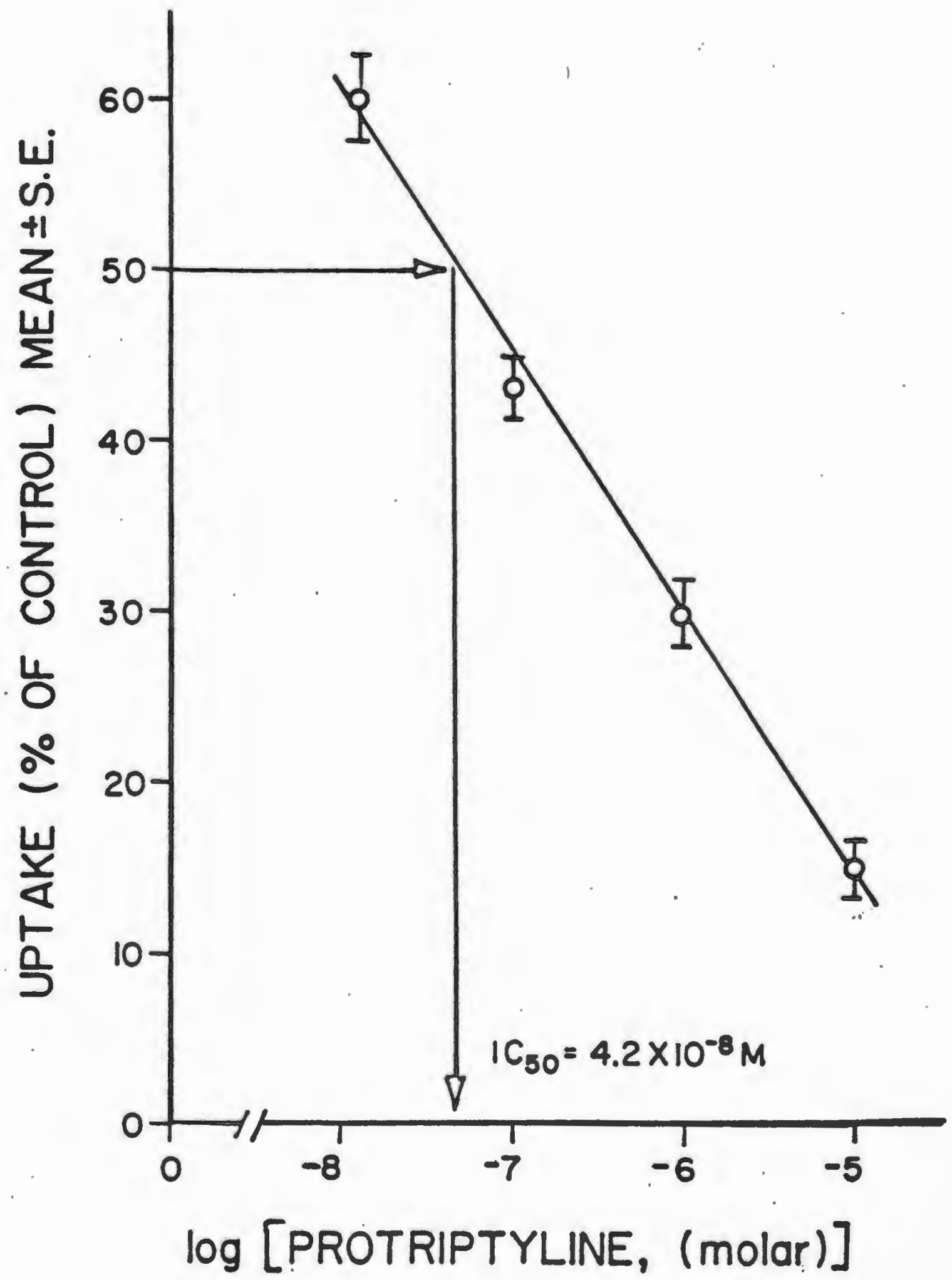


uptake is equivalent to $50 \%$ of control was taken directly by dropping a perpendicular line to the log dose axis.

Use of the logarithmic transformation for biological data has statistical considerations as its basis. Finney (1971) demonstrates how biological data of dose versus response are typically skewed and a logarithmic transformation results in a normal frequency distribution thereby rendering analysis of the data to the application of linear regression and correlation models. The assumption of lin-. earity over a large portion of the dose-response curve is unjustified except for rough approximations (Goldstein, 1967). The use of more accurate methods of determining values of inhibitory constants such as probit analysis can be used for critical experiments. The objectives of these experiments are most conveniently served by the graphic method described.

The results of calculated $I_{50}$ 's are compared with published results for serotonin, norepinephrine and dopamine in tables 7,8 and 9 respectively. Review of these results indicates that the obtained values correlate well with published values. The generalizations described in the literature review for the uptake blocking characteristics of tertiary versus secondary amines is supported by the data summarized in tables seven through nine. Tertiary amines appear more effective in blocking serotonin uptake whereas secondary amines are potent inhibitors of norepinephrine uptake. There appears to be no obvious separation of effects 
Table 7. Norepinephrine uptake $I_{5}{ }^{\prime} s^{*}$ for representative secondary and tertiary amines of the tricyclic antidepressant class of drugs.

\begin{tabular}{|c|c|c|c|c|c|}
\hline PRO & DMI & IMI & AMI & DOX & Reference \\
\hline & 0.03 & 0.2 & & & Ross and Renyi, 1967a \\
\hline & 0.03 & 0.15 & & & Shaskan and Snyder, 1970 \\
\hline & 0.05 & 1.0 & 0.055 & 0.65 & Horn et al., 1971 \\
\hline & 0.006 & & & & Carmichael and Israel, 1973 \\
\hline 0.06 & 0.008 & & & & Maxwell et al., 1974 \\
\hline & 0.003 & 0.028 & 0.054 & & Ross and Renyi, 1975 \\
\hline $\begin{array}{l}0.043 \\
(4)^{3}\end{array}$ & $\begin{array}{c}0.075 \\
(2)^{2}\end{array}$ & $(4)$ & $\frac{0.1}{(2)}$ & $\begin{array}{l}0.51 \\
(2)\end{array}$ & $\begin{array}{l}\text { This study } \\
\text { (number of experiments) }\end{array}$ \\
\hline
\end{tabular}

* $\mathrm{IC}_{50}$ 's expressed in micromolar (uM) 
Table 8. Serotonin uptake $I_{50} \mathrm{~s}^{*}$ for representative secondary and tertiary amines of the tricyclic antidepressant class of drugs.

secondary

\begin{tabular}{|c|c|c|c|c|c|}
\hline PRO & DMI & IMI & AMI & DOX & References \\
\hline \multirow{5}{*}{2.3} & 0.285 & & 0.49 & & Horn and Trace, 1974 \\
\hline & 1.1 & 0.14 & 0.18 & & Ross and Renyi, 1975 \\
\hline & 2 & 0.5 & 0.5 & & Squires, 1974 \\
\hline & 4 & & & & Ross and Renyi, 1969 \\
\hline & 5.8 & 0.5 & & & Carlsson, 1970 \\
\hline 8.3 & 2.7 & & & & Shaskan and Snyder, 1970 \\
\hline 2.7 & 3.3 & 0.3 & 0.67 & & Buus Lassen et al., 1975 \\
\hline 4 & 6.0 & 0.6 & 0.8 & & Kannengiesser et al., 1973 \\
\hline$(4)$ & $(2 \cdot 5)$ & $(4)$ & -- & $2 \cdot 5$ & $\begin{array}{l}\text { This study } \\
\text { (number of experiments) }\end{array}$ \\
\hline
\end{tabular}

* IC 50 's expressed in micromolar (uM) 
Table 9. Dopamine uptake $\mathrm{IC}_{50}{ }^{\prime} \mathrm{s}^{*}$ for representative seconday and tertiary amines of the tricyclic antidepréssant class of drugs.

\begin{tabular}{|c|c|c|c|c|c|}
\hline PRO & DMI & IMI & AMI & DOX & References \\
\hline & 80 & & & & Ross and Reny1, 1967 \\
\hline 6 & 50 & 8 & 4 & 25 & Horn et al., 1971 \\
\hline $\left.\begin{array}{l}5 \\
4\end{array}\right)$ & $\begin{array}{l}50 \\
(2)\end{array}$ & $\begin{array}{l}16 \\
(4)\end{array}$ & $\left(\begin{array}{l}4 \\
2\end{array}\right)$ & -- & $\begin{array}{l}\text { This study } \\
\text { (number of experiments) }\end{array}$ \\
\hline
\end{tabular}

* $\mathrm{IC}_{50}$ 's expressed in micromolar (uM) 
of either secondary or tertiary amines with respect to the inhibition of dopamine uptake.

x) Protriptyline - acute time course

The results of 6 animals in each treatment group given $20 \mathrm{mg} / \mathrm{kg}$ protriptyline 1.p. are presented in figure 10. A one-way analysis of variance applied to the data could not demonstrate the differences seen in this figure to be statistically significant. The data from an additional twelve animals were combined for the control and 6 hour treated groups. The results of a comparison of treatment means using a two-tailed t-test demonstrated significance at the P.05 level. Variability in this system has been shown to be high throughout preliminary investigations of intraperitoneal administration of these drugs with subsequent testing in-vitro. It is important to note that the number of subjects in the acute as well as the subacute study represent individual animals. The variability of the steadystate levels of tricyclic antidepressants in plasma has been shown to be high (Asberg et al.,1971) and this phenomenon may be reflective of the high variability observed in these results. In any event, there appears to be an effect of blocking the uptake of I-NE on acute administration of the drug after six hours.

xi) Protriptyline - subacute time course

Preliminary investigations using a dosage schedule of $20 \mathrm{mg} / \mathrm{kg}$ daily resulted in the death of a number of animals within 8 to 14 days. In virtually all cases of death, 


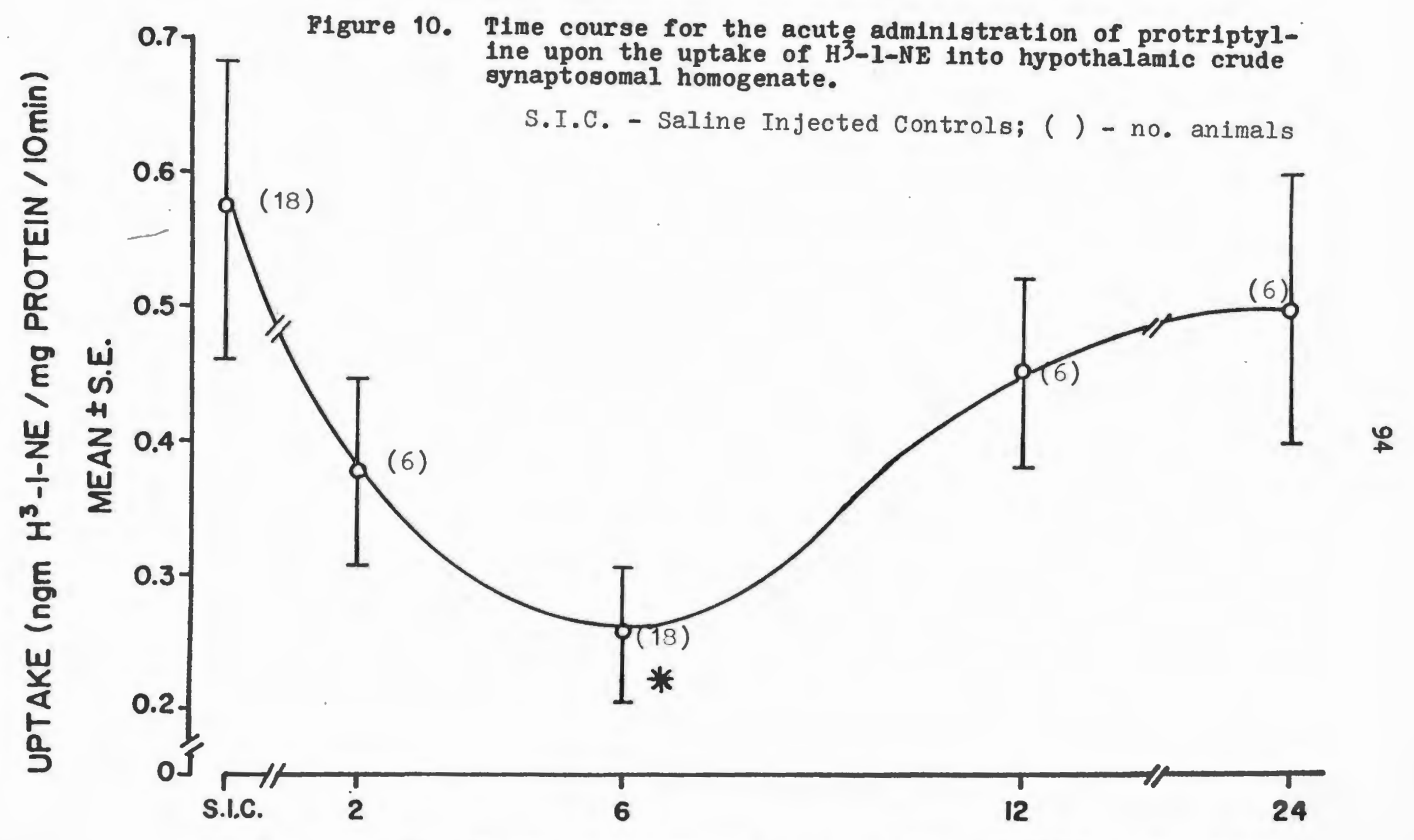

HOURS POST ADMINISTRATION OF PROTRIPTYLINE (20mg/kg I.P.) 
distension of the abdomen was obvious. Autopsies performed on the animals revealed the lower intestine to be filled with gas whereas the upper intestine and stomach was filled with partially digested food. It was concluded that pronounced anticholinergic activity of the drug resulted in paralysis of peristalsis with death due to starvation. Gross behavioral observations of the animals prior to death showed progressive weakening with diminished locomotor activity. The dose employed for the subacute and chronic study was reduced from 20 to $10 \mathrm{mg} / \mathrm{kg}$ to insure the survival of the animals during the chronic study and provide a source for comparison to the subacute study.

Figure 11 illustrates the results obtained from 10 animals per condition administered $10 \mathrm{mg} / \mathrm{kg}$ protriptyline i. o. Once again application of an analysis of variance to the data did not result in statistically significant results. The reduction of uptake observed at 2 hours was $18 \%$ of saline injected controls (S.I.C.). Application of a number of statistical methods including winsorizing and Trimming (Winer,1971) did not result in establishing differences between treatment means. The results then can only be taken as suggestive of an effect at 2 hours.

Ten metabolites for the tertiary amine, imipramine, have been identified and the degree of catabolism of all but the conjugated species have been studied in-vitro using a rat liver microsomal preparation (Bickel,1966). The tertiary amine is catabolized to its corresponding secondary 
Figure 11. Time course for the sub-acute administration of protriptyline upon the uptake of $\mathrm{H}^{3}-\mathrm{I}-\mathrm{NE}$ into hypothalamic crude synaptosomal homogenate.

$$
\begin{aligned}
& \text { S.I.C. - Saline Injected Controls } \\
& N=10
\end{aligned}
$$

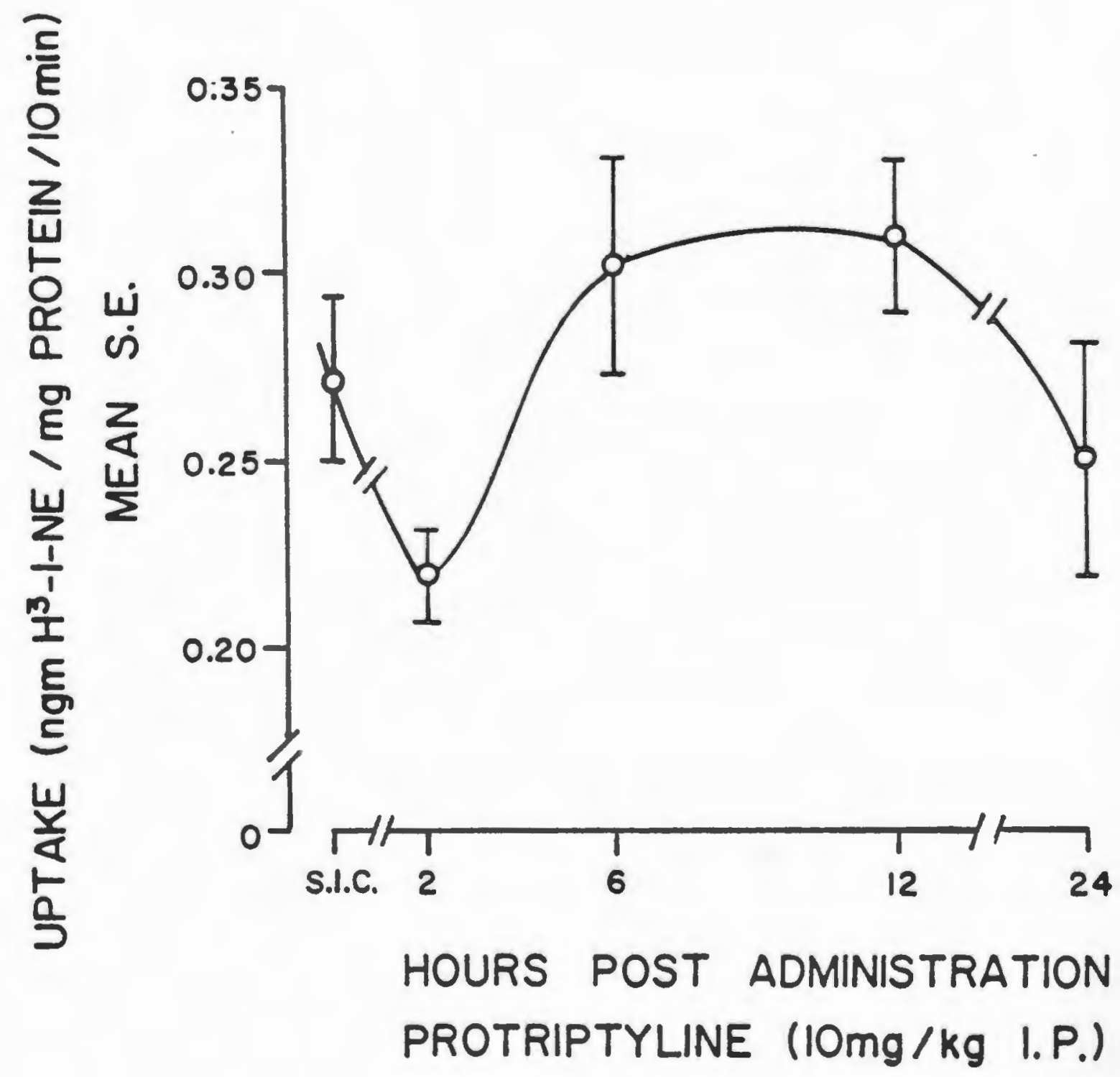


amine and this represents the only major metabolite formed in-vitro. The secondary amine attains peak plasma levels within two hours. Subsequent metabolism of the secondary amine to a primary amine or N-oxidation or ring hydroxylation is less than $10 \%$ of the total substrate concentration within the parameters of the in-vitro test. These results may reflect the metabolism in-vivo and may account for the time course observed in the acute study.

Subacute administration, however, does not lead to a clearly interpretable time course for the effect of uptake inhibition. In fact, variability is extremely high at this point with noticeable, although not statistically significant, increases in uptake above controls which may suggest a rebound phenomenon of the neuronal ending to re-capture lost neurotransmitter. The subacute data of figure 11 can be interpreted in another way. Instead of postulating a clear-cut but relatively ineffective decrease in uptake at two hours as well as a small rebound increase above controls at six and twelve hours, it is possible to view the results as simply no effect after six days of drug. The former view has much conjecture associated with it, the latter is more nearly that necessarily obtained by strict statistical interpretation of the results.

xii) Protriptyline - chronic time course

The results of chronic (18 days) administration of protriptyline upon the uptake of 1-NI into hypothalamic CSH are depicted in figure 12. Application of a one-way analysis 
Figure 12. Time course for the chronic administration of protriptyline upon the uptake of $\mathrm{H} 3-1-\mathrm{NE}$ into hypothalamic crude synaptosomal homogenate.

$$
\begin{aligned}
& \text { S.I.C. - Saline Injected Controls } \\
& \mathbb{N}=5
\end{aligned}
$$

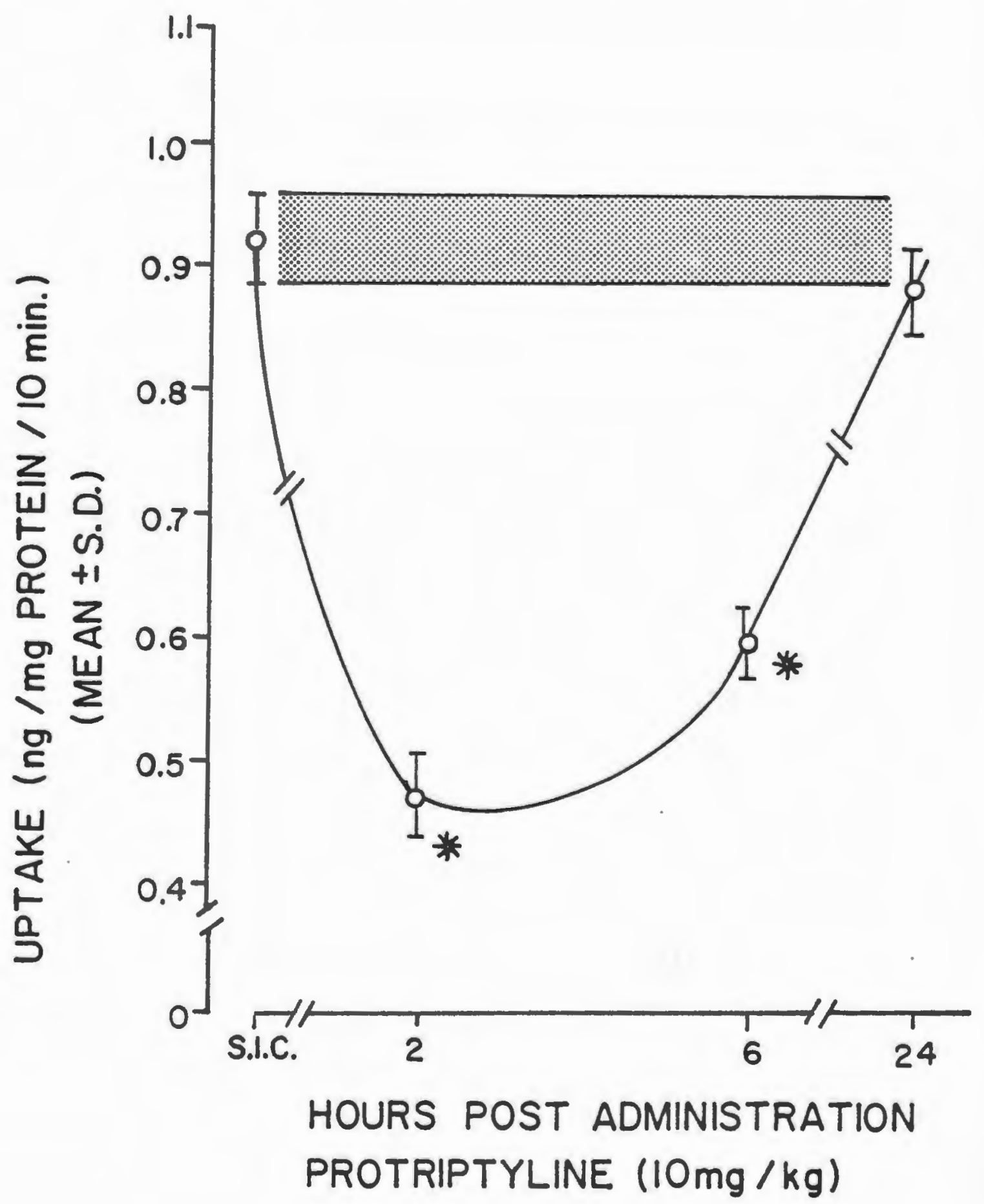


of variance resulted in rejection of the hypothesis of equal means $\left(F_{3,16}=10.52, P .01\right)$ and the assumption of homogeneity of variance was not violated as determined by an $F_{\max }$ test. Subsequent comparison of the treatment means was performed using the Student-Neuman-Keuls multiple range test and demonstrated statistically significant differences at P. 01 for control versus two hours, control versus six hours and twenty-four versus two hours. Treatment at twenty-four versus six hours was shown to be significant at $P .05$, whereas, no statistically significant differences could be demonstrated for either the two versus six hour or control versus twent-four hour treatment groups.

The measure of dispersion about the means reoresented in figure twelve are standard deviations and not standard errors of the means as used in the acute and subacute figures. Although twenty-five animals were used in each treatment condition, the effect of pooling allows them to be represented only as an $n_{i}=5$. Even with relatively low $n_{i}$ compared with previous studies, the data obtained are so precise that it may be concluded that the effect of pooling serves to mask the individual variability considerably.

One additional point worthy of discussion is that of the variation observed between the control groups of the acute, subacute and chronic studies. The weights (and ages) of the animals used in each of these studies varied considerably because selection of the animals used in these studies was based primarily upon animal availability. In the acute 
group the animals weighed approximately 300 grams and the uptake value for the controls in that group was approximately 0.6 as indicated in figure 10. The subacute study employed animals weighing 450 grams and the control uptake value of 0.3 can be observed from figure 11. Finally, the chronic group employed 200 gram animals and their control values were 0.9 as depicted in figure 12. It is easy to conclude that uptake is inversely proportional to the age or weight of the animal. Although each group had its own control, a good argument can be made for the inappropriateness of comparing the results between groups because two variables are known to differ i.e. treatment and the age of the animals. The argument is difficult to refute on theoretical grounds and no attempt to do so will be persued. Instead, these experiments will be repeated with post-pubertal animals prior to publication.

A comparison of the maximal percent of uptake inhibition with respect to their own saline injected controls in the acute, subacute and chronic studies are 56, 18 and 48 percent, respectively. If the consideration noted in the preceding paragraph turns out to be unjustified, then these values alone suggest that uptake inhibition is prominant during either acute or chronic administration and not nearly as effective during subacute administration of protriptyline. The results may suggest that chronic adm nistration of protriptyline results in a long lasting inhibition of the transport carrier for 1-NE uptake into the hypothalamic CSH, 
unlike the results of the subacute experiment. Since uptake after subacute administration may be considered neither prolonged nor unidirectional, it appears that therapeutic effectiveness would not be anticipated during subacute administration and this reflects the clinical picture very accurately. The prolonged effect upon chronic administration appears predictive of a therapeutic response and is clearly temporally related to the therapeutic effect observed clinically. These results support the hypothesis that tricyclic antidepressants may work by blocking re-uptake of endogenous neurotransmitter in-situ. 
102

CHAPTER IV: GENERAI DISCUSSION 
A. Interpretive constraints

One of the principal responsibilities of a scientist is to attempt to remove or at least counter-balance the bias which permeates his or her line of investigation. A good deal of bias is corrected for with the proper use of controls but much bias remains at the level of assumptions made prior to the experimental design. Three assumptions have been made and are discussed here to alert the reader to the limitations of the interpretations made both in the preceding chapter and the conclusions to follow. The first deals with the assumption of unidirectional transmembranous flux of neurotransmitter into synaptosomes and how this phenomenon may confound the measurement of uptake. The second assumption is that of no effect of endogenous neurotransmitter levels upon uptake. Finally the assumption of equivalent protein binding of tricyclic antidepressants to physiological proteins present in the incubation medium is discussed.

Wuch of the experimental work reported here is concerned with the characterization of the synaptosomal system used to study the effects of intraperitoneally administered drugs by subsequent in-vitro testing. The characterization lends credibility to the proper functioning of the system in that it clearly demonstrates the degree to which this system compares with those used by other investigators. However, in no way does it resolve critical issues with respect to the transport of 1-norepinephrine into synaptosomes.

One such issue remaining unresolved is that regarding 
the extent to which neurotransmitter exchange processes occur and may confound the measurement of an estimate of net uptake. An approach to resolving this question may be to measure the specific activity of neurotransmitter located in the incubation medium, the synaptosomal cytoplasmic as well as vesicular fractions separately both before and after incubation at physiological temperatures. The results of these experiments may indicate whether endogenous neurotransmitter stored within vesicles, the cytoplasm or that resulting from de novo synthesis somehow contributes toward an exchange process.

Another unresolved question is that of the relationship between endogenous neurotransmitter content and the uptake of exogenously applied neurotransmitter. A review of the literature suggests that endogenous biogenic amine levels are lowered upon chronic administration and turnover appears to be increased. These effects appear intuitively concordant with what one might expect of uptake inhibitors, however, this interpretation presupposes that the effect of uptake inhibition is responsible for the lowered levels. The evidence comes from demonstrating the uptake inhibitory characteristics of the tricyclic antidepressants as measured in-vitro. Lowered levels of endogenous amines may be responsible for a decrease in uptake and if this is so, the inhibition of uptake observed may be an indirect effect of the drug. This important question seems experimentally approachable by comparing the effects of biogenic amine 
synthesis inhibitors upon uptake measured both in-vitro and after parenteral administration with subsequent testing invitro. The data presented in this thesis with respect to neurotransmitter refilling of previously emptied synaptosomes suggests that levels of neurotransmitters can affect uptake. Snyder and Coyle (1969) reported a decrease in uptake of norepinephrine in animals previously treated with reserpine as opposed to saline injected controls. In addition it is important to realize that compartmentation of neurotransmitter within the neuronal ending may affect uptake differentially. Investigation of the pharmacodynamics of the tricyclics with respect to uptake inhibition should consider the effect of pool sizes as well as total levels of endogenous neurotransmitters.

The potencies of the tricyclics as uptake inhibitors are taken from concentration terms in-vitro whereas it has been shown that the binding of tricyclic antidepressants to plasma proteins is variable and does not reflect a clear separation of effects between secondary and tertiary amines (Borga et al., 1969). The in-vitro experiments therefore necessitate an assumption of equivalent binding to plasma proteins for a direct domparison of potency to be considered legitimate. Protriptyline and desmethylimipramine are bound to plasma proteins leaving only 8.0 and 8.4 percent free in the plasma, respectively. Imipramine and amitriptyline are free in plasma to the extent of 4.2 and 3.6 percent, respectively. Nortriptyline however, is 94.5 percent 
bound. Since free drug is considered to be pharmacologicalIy active whereas that bound to plasma proteins are inactive, the relative degree of binding may be an important variable in ascertaining meaningful potency ratios in-vitro and invivo (Glassman et al., 1977). Although much work has been done in an attempt to correlate blood plasma levels with the therapeutic effect (Alexanderson et al., 1973), little has been reported on the relationship between the concentration of free drug and clinical outcome (Glassman et al., 1973, Nuscettola et al., 1978).

B. Alternative mechanisms

Caution must be taken at this time to refrain from concluding that tricyclics mediate their therapeutic effects by uptake inhibition or blockade. The spectrum of pharmacological activity of the tricyclic antidepressants is not limited to uptake blockade. In fact, two additional biochemical effects are being suggested as possible determinants of the therapeutic effect of the tricyclics. Edwards and Burns (1974) demonstrated the ability of tricyclics to block MAO found in human platelets and Roth and Gillis (1974) reported an MAO inhibitory effect of tricyclics in rabbit brain mitochondria.

Alternatively, tricyclics possess reasonable strong anticholinergic effects demonstrated both peripherally, using antagonism of carbachol contraction of the guinea pig ileum, and centrally by displacements of $\mathrm{H}^{3}$-quinuclidinyl benzylate, a potent muscarinic antagonist, from homogenates 
of rat brain (Sayers and Burki, 1976; Snyder and Yammamura, 1977). Janowsky et al. (1972) review the evidence for the efficacy of anticholinergics in the treatment of depression.

In addition to the effects suggested as components of the thymoleptic effect, tricyclics possess other therapeutic effects when administered in doses below that required for the treatment of depression. Wilkerson (1978) reported o the ability of tricyclics to reverse ouabain induced ventricular arrhythmias in dogs at doses below that used for the treatment of depression. Similarly, protriptyline has been demonstrated to be effective in the treatment of hypersomnia and the narcolepsy-catalepsy syndrome after a single bedtime dose of $10 \mathrm{mg}$ (Schnidt et 21., 1977).

Other clinically effective antidepressants exhibit different pharmacological profiles than the tricyclics. Mianserin is not a very potent inhibitor of catecholamine or serotonin uptake (Goodlet et al., 1977). Nomifensine possesses weak anticholinergic activity but is very potent inhibitor of dopamine uptake and has clinical psychostimulatory properties (Funt et al., 1974).

Comparisons of the performance of tricyclics as well as other clinically effective antidepressants in a number of biochemical systems may serve to demonstrate the extent to which each property (i.e. anticholinergic, uptake inhibition, receptor binding affinity, MAO inhibition, etc.) may either contribute to or be predictive of either the thymoleptic effect or side effects. These comparisons may suggest a 
role for specific neurotransmitters or a balance of neurotransmitters in depression or any of the other clinical entities in which tricyclics are or can be used.

One type of comparative technique is demonstrated in table 10. The table was compiled from all in-vitro data reviewed in tables $7-9$ as well as the anticholinergic data of Shein and Smith (1978). Inclusion of the anticholinergic potencies was based upon availability of comparative data for the particular tricyclics investigated and reported in this thesis. Unfortunately, comparative data across a number of seconday and tertiary amines are not available for the other properties mentioned.

Before proceeding it is important to point out that a similarly compiled table may possess a great deal more predictive value if each of the biochemically determined potencies were measured after chronic parenteral administration of the drug. As Inch et al. (1973) point out, the comparison of drug effects in-vivo can only be considered valid if the time course for the effect of each drug is determined and the time representing the optimal effects are used to determine potencies. This would necessitate performing a time course for each drug after chronic administration. The in-vitro comparisons then are performed with the understanding that they might not reflect the actual relative potencies which may occur upon chronic administration of the drug, however, it represents a starting point and a source of comparison for data which will no doubt become 
Table 10. Summary of relative and absolute potencies of representative secondary and tertiary amines of the tricyclic antidepressant (TCA) class of drugs.

\begin{tabular}{|c|c|c|c|c|c|c|c|c|c|c|c|}
\hline \multirow{2}{*}{$\begin{array}{l}\text { Sub } \\
\text { class } \\
\text { of } \\
\text { TCA }\end{array}$} & \multirow[b]{2}{*}{ DRUG } & \multicolumn{4}{|c|}{ POTENCIES } & \multicolumn{6}{|c|}{ RELATIVE POTENCY RATIOS } \\
\hline & & 5-HT & NE & $\mathrm{DA}$ & ** R Ach P & $\frac{5-\mathrm{HT}}{\mathrm{NE}}$ & $\frac{\mathrm{DA}}{\mathrm{NE}}$ & $\frac{5-\mathrm{HT}}{\mathrm{DA}}$ & $\frac{\mathrm{Ach}}{5-\mathrm{HT}}$ & $\frac{\mathrm{Ach}}{\mathrm{NE}}$ & $\frac{\mathrm{Ach}}{\mathrm{DA}}$ \\
\hline \multirow{2}{*}{\begin{tabular}{|l|}
$S$ \\
E \\
C \\
O \\
N \\
D \\
A \\
B \\
\end{tabular}} & PRO & $\begin{array}{c}5.3 \pm 1.4 \\
+(5)\end{array}$ & $\begin{array}{c}.015 \pm .01 \\
(2)\end{array}$ & $\begin{array}{c}5.5 \pm .5 \\
(2)\end{array}$ & 2.18 & 104 & 108 & .964 & $\begin{array}{c}.411 \\
\ddagger(0.9 \%)\end{array}$ & $\begin{array}{c}42.7 \\
(98.1 \%) \\
\end{array}$ & $\begin{array}{l}.396 \\
(0.9 \%)\end{array}$ \\
\hline & DMI & $\begin{array}{c}3.3 \pm .67 \\
(9)\end{array}$ & $\begin{array}{c}.025 \div 01 \\
(7)\end{array}$ & $\begin{array}{c}60 \pm 10 \\
(3)\end{array}$ & 0.33 & 132 & 2600 & .055 & $\begin{array}{c}.100 \\
(0.7 \%)\end{array}$ & $\begin{array}{c}13.2 \\
(99.2 \%) \\
\end{array}$ & $\begin{array}{l}.006 \\
(0.04 \%) \\
\end{array}$ \\
\hline \multirow{3}{*}{$\begin{array}{l}T \\
\mathbf{E} \\
\mathbf{R} \\
\mathbf{T} \\
\mathbf{I} \\
\mathrm{A} \\
\mathrm{R} \\
\mathbf{Y}\end{array}$} & IMI & $\begin{array}{c}.33 \pm .09 \\
(5)\end{array}$ & $\begin{array}{c}75 \pm .45 \\
(5)\end{array}$ & $\begin{array}{r}12 \pm 4 \\
(2)\end{array}$ & 1.00 & .440 & 16.0 & .028 & $\begin{array}{c}3.03 \\
(68.6 \%)\end{array}$ & $\begin{array}{c}1.30 \\
(29.5 \%\end{array}$ & $\begin{array}{l}.083 \\
(1.8 \%)\end{array}$ \\
\hline & AMI & $\begin{array}{c}.53 \pm .1 \\
(5)\end{array}$ & $\begin{array}{c}.07 \pm .01 \\
(4)\end{array}$ & $\begin{array}{c}4 \pm 0 \\
(2)\end{array}$ & 4.17 & 7.57 & 57.0 & .133 & $\begin{array}{c}7.86 \\
(11.4 \%)\end{array}$ & $\left|\begin{array}{c}59.6 \\
(87.1 \%)\end{array}\right|$ & $\begin{array}{l}.959 \\
(1.4 \%)\end{array}$ \\
\hline & DOX & $\begin{array}{c}2.9 \pm .4 \\
(2)\end{array}$ & $\begin{array}{c}.58 \pm .7 \\
(2)\end{array}$ & $\begin{array}{l}25 \\
(1)\end{array}$ & 0.42 & 5.00 & 43.0 & .116 & $\begin{array}{c}.145 \\
(16.3 \%)\end{array}$ & $\begin{array}{c}.724 \\
(81.7 \%)\end{array}$ & $\begin{array}{l}.017 \\
(1.9 \%)\end{array}$ \\
\hline \multicolumn{12}{|c|}{ 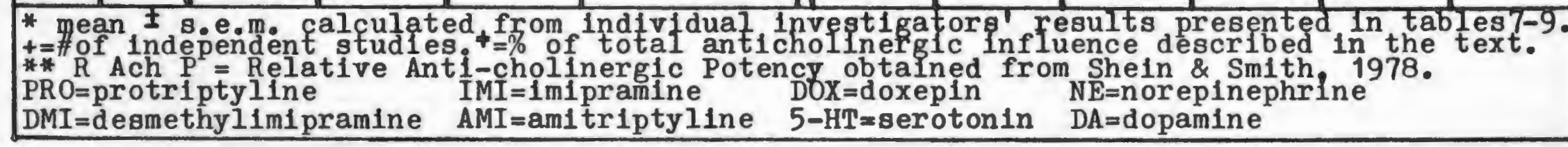 } \\
\hline
\end{tabular}


available with future research.

To the left of the vertical double line in table 10 are the uptake inhibitory potencies of the tricyclics with respect to three neurotransmitters as well as the relative (to imipramine) anticholinergic potencies. The relative potency ratios for serotonin, norepinephrine and dopamine, referred to as the biogenic amine ratios, can be compared with one another but the anticholinergic:biogenic amine ratios, in the last three columns of table 10, cannot be compared directly with the biogenic amine ratios simply because a different response measure was used in determining the anticholinergic influence. The values obtained for the anticholinergic:biogenic amine ratios become more meaningful when viewed as a percent of the total anticholinergic influence with respect to each of the biogenic amines. The values placed within parentheses directly below the anticholinergic: biogenic amine ratios were obtained by summing the three ratios for a single drug and expressing each ratio as a percent of the total.

Several portions of this table demonstrate interesting correlations with known effects of tricyclics. Based upon the biogenic amine comparisons in the first three columns to the right of the double line, there appears to be no difference between the effects of amitriptyline and doxepin. Clinical studies do not demonstrate any clear differences between doxepin and amitriptyline as antidepressants (Beck, 1973). Doxepin has been reported to be well tolerated by 
older patients because of fewer incidences of anticholinergic side effects. This difference is clearly reflected in the potencies of anticholinergic effects where amitriptyline is ten times more potent than doxepin. This may suggest that the anticholinergic properties are not important for the clinical therapeutic effect. Cairncross et al. (1963) suggested that the anticholinergic effect was important for therapeutic activity but Blackwell et al. (1972) concluded that the anticholinergic effect was related more to the sedative properties of the drugs. This is difficult to reconcile since stimulation of locomotor activity, a behavioral response measure which could be viewed as an inverse indicator of sedation, has been shown to be centrally mediated by anticholinergic drugs (Aquilonius et al., 1972). It may be that the difference in anticholinexgic potency is only reflected in the periphery with these two drugs. The particular proportional indluences upon the biogenic amine systems may serve to mask any psychostimulatory properties these drugs possess if they were purely anticholinergic. This may occur as a result of the lack of proportional influences within the periphery that these neurotransmitter systems may possess within the central nervous system. A look at the anticholinergic:biogenic amine ratio "percent" figures of table 10 suggests that there are no differences in the relative effects of anticholinergic to biogenic amine influences between amitriptyline and doxepin. It may be as a result of this balancing influence that doxeoin 
and amitriptyline are sedative.

On the other end of the pharmacological spectrum is the psychostimulatory effects of protriptyline. Frotriptyline is the only tricyclic antidepressant recommended by a manufacturer for use in anergic patients. Since dopaminergic influences are believed to be responsible for stimulating effects on locomotor activity (Fibiger et al., 1973; Creese and Iversen, 1973; 1975; Tseug and Ioh, 1974; Tseug et al., 1974) and cholinergic-dopaminergic interactions are important as well (Klawans, 1968; Hornykiewicz, 1966; Friehoff \& Alpert, 1973), it seems reasonable to conclude that a cholinergic-dopaminergic interaction may mediate the psychostimulatory effect of this drug. Compared with desmethylimipramine, the percent of anticholinergicdopaminergic interaction is 22 times greater for protriptyline whereas the effects with respect to anticholinergicnorepinephrine or anticholinergic-serotonin influences are norepinephrine or anticholinergic-serotonin influences are comparable. This may reflect the mechanism of psychostimu lation occuring in-vivo.

This approach may offer a reasonable, although admittedly speculative, interpretation for the different effects observed with the clinical use of tricyclics, but most important is the therapeutic effect. Review of the relative potency ratios for biogenic amines demonstrates the common factors which may suggest how all these drugs may work with respect to the neurotransmitters studied. All 
dopamine:norepinephrine ratios are greater than one and all serotonin:dopamine ratios are less than one. Considering only the biogenic amines, this is the only factor common to all tricyclics reflected in table 10. It may be suggested that the relative effects of serotonin with respect to dopamine and norepinephrine with respect to DA are involved in the antidepressant effect of these drugs.

Continuing with the concept that a balance of effects upon critical neurotransmitters may be responsible for the therapeutic effect, these observations are reminisent of the elements of the "permissive hypothesis of depression" (Prange et al., 1974). This hypothesis states that serotonin acts as a gating or controlling influence for the expression of either mania or depression by increased or decreased levels of norepinephrine, respectively. Agents affecting these systems perhaps with respect to a third buffer or reference system such as the dopaminergic system may account for the common dimension required for a unified neurotransmitter hypothesis of affective disorders.

An attempt to explain the mechanism of action of these drugs using only four of possibly scores of neurotransmitters is admittedly premature. Certainly other mechanisms may be responsible for the therapeutic effect both within as well as outside the frame work of the biogenic amine hypothesis.

It is important to nore that the development of the tricyclics came out of investigations with antihistaminic 
drugs are tricyclics possess antihistaninic properties. Research directed towards evaluating these properties may reflect more light on even more complex interactions then we dare to expect. Knowledge of the role of neurotransmitters in behavior is still at its infancy (Baldessarini, 1975). Until more data is accumulated with respect to the pharmacokinetics, physical properties and pharmacodynamics of tricyclic antidepressants, table like that of table 10 represent a way in which leads toward a particular line of investigation may be obtained. A glimmer of hope in unravelling the complex interactions of a number of variables in biological systems is the increasing applications of statistical methods. Techniques of utilizing parametric and nonparametric statistical methods are becoming more accessible and easier to apply. Pattern recognition techniques are available to aid in the analysis of complex interactions using cluster analysis, path analysis, orincipal components and other multivariate approaches (Harper, et al., 1977). C. Conclusion

Should the limitations noted above prove to be negligible, then it would appear that protriptyline blocks the uptake of norepinephrine upon chronic administration whereas when administered sub-acutely uptake blockade is not clearly discernable. These findings demonstrate a temporal correlation of uptake blockade with clinical effectiveness in humans and lends support to both the catecholamine hypothesis and the proposed mechanism of action of tricyclics as uptake 
blockers. In addition it is interesting to note that there is a rislk of attempted suicide in depressives during the initial days of chemotherapy (Beck, 1973) and sub-acute administration displays highly variable effects and perhaps even some potentiation of uptake thereby enhancing the functional depletion of neurotransmitter at the level of the post synaptic receptors. This effect could explain the clinical observation of increased suicidal tendency during initiation of tricyclic chemotherapy.

In support of the sub-acute data obtained is the evidence that twelve hours post-protriptyline using either serotonin or tryptamine as substrate, MAO activity is elevated (Ed Kaiser-personal communication). An increase in uptake provides more substrate for $M A O$ therefore these results appear corroborative of the subacute data presented here.

It appears that the relative anticholinergic properties can be predictive of the psychostimulatory effects of protriptyline. Comparisons of the effects of drugs using in-vitro biochemical techniques may be useful in formulating hypotheses regarding the mechanism of action of the tricyclic antidepressants. The lack of psychostimulatory action of the most potent anticholinergic of the tricyclics, amitriptyline, may be explained by a relative interaction between anticholinergic and dopaminergic influences. 
116

CHAPTER V: REFERENCES 
Adamic. S.: Accumulation of acetylcholine by the rat diaphragm Biochem. Pharmaco1. 19:2445-2451,1970.

Aghajanian, G.K., 3loom, F.E., Lovell, R.A., Sheard, M.H. \& Freedman, D.X.: The uptake of 5-hydroxytryptamine-H3 from the cerebral ventricles: An autoradiographic localization. Biochem. Pharmacol. 15:1401-1403,1966.

Alexanderson, B., Asberg, M. \& Tuck, D.: Relationship between the steady-state plasma concentration of nortriptyline and some of its pharmacological effects. In Biological Effects of Drugs in Relation to Their Plasma Concentration Davies, D.S. \& Erichard, 3.N.C. (eds.) British Pharmacological Society, University Park Press, Baltimore, Md. 191-199,1973.

Anden, N.-E., Dahlstrom, A., Larsson, K., Olson, I. \& Ungerstedt, U.: Ascending monoamine neurons to the telencephaion and diencephalon. Acta Physiol. Scand. $67: 313-326,1966$.

Aquilonius, S.M., Iundholm, B. \& Winbladh, B.: Effects of some anticholinergic drugs on cortical acetylcholine release and motor activity in rats. Europ. J. Pharmacol. 20:224-230,1972.

Arregui, A., Logan, W.J., Bennett, J.P. \& Snyder, S.H.: Specific glycine-accumulating synaptosomes in the spinal cord of rats. Proc. Nat. Acad. Sci. 69:34853489,1972 .

Asberg, M., Cronholm, B. Sjoqvist, F. \& Tuck, D.: The correlation of subjective side-effects with plasma concentrations of nortriptyline. Brit. Med. J. 4: $18-21,1970$.

Asberg, M., Cronholm, B., Sjoqvist, F. \& Tuck, D.: Relationship between plasma level and therapeutic effect of nortriptyline. Brit. Med. J. 3:331-334,1971.

Avakian, O.V. \& Gillespie, J.S.: Uptake of noradrenaline by adrenergic nerves, smooth muscle and connective tissue in isolated perfused arteries and its correlation with vasoconstrictor response. Brit. J. Pharmacol. Chemother. 32:168-184,1968.

Axelrod, J.: 0-Methylation of epinephrine and other catechols in-vitro and in-vivo. Science 126:400-401,1957.

Axelrod, J.: Metabolism of epinephrine and other sympathomimetic amines. Physiol. Rev. 39:751-776,1959.

Axelrod, J., Hertting, G. \& Patrick, R.W.: Inhibition of $\mathrm{H}^{3}$-norepinephrine release by monoamine oxidase inhib- 
itors. J. Pharmacol. 134:325-328,1961a.

Axelrod, J., Hertting, G. \& Potter, I.T.: Effect of drugs on the uptake and release of $\mathrm{H}^{3}$-norepinephrine in the rat heart. Nature 194:297,1962.

Axelrod, J. \& Tomchick, R.: Increased rate of metabolism of epinephrine and norepinephrine by sympathomimetic amines. J. Pharmacol. 130:367,1960.

Axelrod, J., Weil-Malherbe, H. and Tomchick, R.: The physiological disposition of $\mathrm{H}^{3}$-epinephrine and its metabolite metanephrine. J. Pharmacol. Exp. Ther. 127:251-256, 1959.

Axelrod, J., Whitby, I.G. and Hertting, G. psychotropic drugs on the uptake of $\mathrm{H}^{3}$-norepinephrine by tissues. Science 133:383-384,1961b.

Bacq, Z.M., Gosselin, I., Dresse, A. \& Renson, J.: Inhibition of O-methyltransferase by catechol and sensitization to epinephrine. Science 130:453-454,1959.

Balcar, V.J. \& Johnston, G.A.R.: Glutamate uptake by brain slices and its relation to the depolarization of neurons by acidic amino acids. J. Neurobiol. 3:295$301,1972 a$.

Balcar, V.J. \& Johnston, G.A.R.: The structural specificity of the high affinity uptake of l-glutamate and l-aspartate by rat brain sices. J. Neurochem. 19: 2657-2666,1972b.

Balcar, V.J. \& Johnston, G.A.R.: High affinity uptake of neurotransmitters: Studies on the uptake of 1-aspartate, GABA, l-glutamate and glycine in cat spinal cord. J. Neurochem. 20:529-539,1973.

Baldessarini, R.J.: Biogenic amine hypothesis of affective disorders. In The Nature and Treatment of Depression Flack, F.F. \& Draghi, S.C. (eds.) Wiley \& Sons, Inc. New York. 347-385, 1975.

Balzer, H. \& Holtz, P.: Beeinflussung der wirkung biogener amine durch hemmung der aminoxydase. Arch. Exp. Path. Pharmakol. 227:547-558,1956.

Beck, A.T.: The Diagnosis and Management of Depression University of Pennsylvania Press, Phila. 81-122,1973.

Bennett, J.P., Iogan, W.J. \& Snyder, S.H.: Amino acid neurotransmitter candidates: Sodium dependent highaffinity uptake by unique synaptosomal fractions. Science 178:997-999,1972. 
Bickel, M.H.: Metabolism and structure-activity relationships of thymoleptic drugs. In Antidepressant Drugs Garattini, S. \& Dukes, M.N.G. (eds.) Excerpta Medica Foundation, International Congress Series No, 122. 3-9, 1966 .

Blackwell, B. Iipkin, J.O., Meyer, J.H., Kuzma, R. \& Boulter, W.V.: Dose responses and relationships between anticholinergic activity and mood with tricyclic antidepressants. Psychopharmacol. 25:205-217, 1972.

Blaustein, M.P., Johnson, E.M. \& Needleman, P.: Calciumdependent norepinephrine release from pre-synaptic nerve endings in-vitro. Proc. Nat. Acad. Sci. 69: 2237-2240,1972.

Bloom, F.E. \& Iversen, I.I.: Localizing 3H-GABA in nerve terminals of rat cerebral cortex by electron microscopic autoradiography. Nature 229:628-630,1971.

Bogdanski, D.F. and Tissari, A.: Sodium dependent uptake and storage of amines in synaptosomes. Pharmacologist 9: 250,1967 .

Bogdanski, D.F., Tissari, A.H. \& Brodie, B.B.: Role of sodium, potassium, ouabain and reserpine in uptake, storage and metabolism of biogenic amines in synaptosomes. Iife Sci. 1:419-428,1968.

Borga, O., Azarnoff, D.I., Plym Forshell, G. \& Sjoqvist, F.: Plasma protein binding of tricyclic antidepressants in man. Bíochem. Pharmacol. 18:2135-2143,1969.

3radford, H.F.: Isolated nerve terminals as an in-vitro preparation for the study of dynamic aspects of transmitter metabolism and release. In Handbook of Psychopharmacology Plenum Press, New York Iversen, I.L., Iversen, S.D. and Snyder, S.F. $1: 191-252,1975$.

Brodie, B.B. \& Beaven, M.A.: Neurochemical transducer systems. Med. Exp. 8:320-351,1963.

Brown, G.I.: The Croonian Iecture 1964: The release and fate of the transmitter liberated by adrenergic nerves. Proc. Roy. Soc. Biol. 162:1-19,1965.

Burgen, A.S.V. \& Iversen, I.I.: The inhibition of noradrenaline uptake by sympathomimetic amines in the rat isolated heart. Brit. J. Pharmacol. Chemother. $\underline{25}: 34-49,1965$.

Burn, J.H.: The action of tyramine and ephedrine. 
J. Pharmacol. Exp. Ther. 46:75-95,1932.

Burn, J.H.: The mechanism of the action of chemical substrates at nerve endings and the part played by peripheral ganglia. Acta Physiol. Scand. 29:40-49,1953.

Burnstock, G., McClean, J.R. \& Wright, M.: Noradrenaline uptake by non-innervated smooth muscle. Brit. J. Pharmacol. 43:180-189, 1971 .

Burton, R.M.: Gangliosides and acetylcholine of the central nervous system III. The binding of radioactive acetylcholine by subcellular particles of the brain. Int. J. Neuropharmacol. 3:13-21,1964.

Cairncross, K.D.:, Gershon, S. \& Gust, I.D.: Some aspects of the mode of action of imipramine. J. Neuropsychiatr. $4: 224-231,1963$.

Carlsson, A., Hillarp, N.A. \& Waldeck, B.: Analysis of the $\mathrm{Mg}^{++}-\mathrm{ATP}-d e p e n d e n t$ storage mechanisms in the amine granules of the adrenal medulla. Acta Physiol. Scand. 59Suppl.215, 1963.

Chirigos, M.A., Greengard, P. \& Udenfriend, S.: Uptake of tyrosine by rat brain in-vitro. J. Biol. Chem. 235: 2075-2079, 1960.

Clarke, D.E., Jones, C.J. \& Iinley, P.A.: Histochemical flourescence studies on noradrenaline accumulation by uptake 2 in the isolated rat heart. Brit. J. Pharmacol. 37: 1-9,1969.

Cleland, W.W.: The statistical analysis of enzyme kinetic . data. Advan. Enzymol. 29:1-32,1967.

Cohen, S.R. and Lajtha, A.: Amino acid transport. In Handbook of Neurochemistry Lajtha, A. (ed.) Plenum Press, New York 7:543-572, 1972.

Colburn, R.W., Goodwin, F.K., Murphy, D.I., Bunney, W.E. \& Davis, J.M.: Quantitative studues of norepinephrine uptake by synaptosomes. Biochem. Pharmaco1. 17:957$964,1968$.

Cooper, J.R., Bloom, F.E. \& Roth, R.H.: Blochemical Basis of Neuropharmacology Second Edition; Oxford Press p. 126,1974 .

Corne, S.J. \& Graham, J.D.P.: The effect of inhibition of amine oxidase in-vivo on administered adrenaline, noradrenaline, tyramine and serotonin. J. Physiol. 135:339-349,1957. 
Cornish-Bowden, A. \& Eisenthal, R.: Statistical considerations in the estimation of enzyme kinetic parameters by the direct linear plot and other methods. Biochem. J. $139: 721-730,1974$.

Costa, E.: Turnover rate of neuronal monoamines: pharmacological implications. In The Present Status of Psychotrooic Drugs: Proceedings of the International Congress of the collegium Internationale NeuroDsych-oharmacologium Cerletti, C. \& Bove, F.J. Excerpta Medica Foundation, Amsterdam. 11-35,1969.

Costa, E. \& Meek, J.I.: Regulation of biosynthesis of catecholamines and serotonin in the CNS. Ann. Rev. Pharmacol. 14:491-511,1974.

Coyle, J.T. \& Snyder, S.H.: Catecholamine uptake by synaptosomes in homogenates of rat brain: Stereospecificity in different areas. J. Pharmacol. Exp. Ther. 170:221231,1969 .

Creese, I. \& Iversen, S.D.: Blockade of amphetamine induced motor stimulation and stereotypy in the adult following neonatal treatment with 6-hydroxydopamine. Brain Res. 55:369-382,1973.

Creese, I. \& Iversen, S.D.: The pharmacological and anatomical substrates of the amphetamine response in the rat. Brain Res. 83:419-436,1975.

Davis, J.M.: Theories of biological etiology of affective disorders. Int. Rev. Neurobiol. 12:145-175,1970.

Davis, J.M., Goodwin, F.K., Bunney, W.E. Jr., Murphy, D.I. \& Colburn, R.W.: Effects of ions on uptake of norepinephrine by synaptosomes. Pharmacologist 2:184,1967.

Dengler, H.J.: Effects of drugs on monoamine uptake in isolated tissue. In Proceedings of the second International Pharmacology Meeting. Prague, 1963 Koelle, G.3. Douglas, W.W. \& Carlsson, A. Pergamon Press, Oxford. $\underline{3}: 261-275,1965$.

Dengler, H.J., Michaelson, I.A., Splegel, H.E. \& Titus, E.: Int. J. Neuropharmacol. 1:23-38,1962.

Dengler, H.J., Spiegel, H.E. \& Titus, E.O.: Effects of drugs on uptake of isotopic norepinephrine by cat tissues. Nature 191:816-817,1961.

Dowd, J.E. \& Riggs, D.S.: A comparison of estimates of Michaelis-Menten kinetic constants from various linear transformations. J. Yiol. Chem. 240:863-869,1965.

Dowdall, M.J. and Simon, E.J.: Comparative studies on 
synaptosomes: Uptake of (N-Me- $\left.\mathrm{F}^{3}\right)$ choline by synaptosomes from squid optic lobes. J. Neurochem. 21:969$982,1973$.

Draskoczy, P.R. \& Trendelenburg, U.: Intra-neuronal and extra-neuronal sympathomimetic amines in the isolated nictitating membrane of the cat. J. Pharmacol. Exp. Ther. 174:290-306,1970.

Edwards, D.J. \& Burns, M.O.: Effects of tricyclic antidepressants upon human platelet monoamine oxidase. Iife Sci. 15:2045-2058, 1974.

Ehinger, B.: Cellular location of the uptake of some amino acids into the rabbit retina. Brain Res. 46:297-311,1972.

Ehinger, B. \& Sporrong, B.: Neural and extra-neural localization of noradrenaline in the rat heart after perfusion at high concentration. Experientia 24:265-266, 1968.

Eisenthal, R. \& Cormish-Bowden, A.: The direct linear plot: A new graphical procedure for estimating kinetic parameters. Biochem. J. 139:715-720,1974.

Elliot, X.A.C. \& Henderson, N.: Factors affecting acetylcholine found in excised brain. Amer. J. Physiol. $165: 364-374,1951$.

Elmore, D.T., Kingston, A.E. \& Shields, D.3.: The computation of velocities and kinetic constants of reactions, with particular reference to enzyme-catalysed processes. J. Chem. Soc. Pt.2:2070-2078,1963.

Euler, U.S. von \& Iishajko, F.: Uptake of I- and D- isomers of catecholamines in adrenergic nerve granules. Acta Physiol. Scand. 60:217-222,1964.

Farnebo, I.0. \& Malmfors, T.: Histochemical studies on the uptake of noradrenaline and alpha-methylnoradrenaline in the perfused rat heart. Europ. J. Pharmacol. 5: 313-320, 1969 .

Fibiger, H.C., Fibiger, H.P. \& Zis, A.P.: Attenuation of amphetamine induced motor stimulation and stereotypy by 6-hydroxydopamine in the rat. Brit. J. Pharmacol. 47:683-692, 1973 .

Finney, D.J.: Probit Analysis Third Edition Cambridge University Press, London 8-17, 1971.

Fischer, J.E., Kopin, I.J. and Wurtman, R.I.: Effects of lumbar sympathectomy on the uterine uptake of catechol- 
amines. Nature 203:938-939,1964.

Friedhoff, A.J. \& Alpert, N.: A dopaminergic-cholinergic mechanism in the production of psychotic symptoms. Biol. Psychiatr. 6:165-169,1973.

Iuxe, K. \& Ungerstedt, U.: Localization of 5-hydroxytryptamine uptake in rat brain after intraventricular injection. J. Pharm. Pharmacol. 19:335-337,1967.

Giese, J., Ruther, E. \& Matussek, N.: Quantitative estimation of $\mathrm{H}^{3}$-norepinephrine and its metabolites by thinlayer chromatography. Iife Sci. 6:1975-1982,1967.

Gillespie, J.S.: The role of receptors in adrenergic uptake. In Adrenergic Neurotransmission Wolstenholme, G.E.W. \& O'Connor, H. (eds.) Ciba Foundation Study Group No. 33. Churchill, Iondon. 61-72,1968.

Gillespie, J.S.: Uptake of noradrenaline by smooth muscle. Brit. Med. Bull. 29:136-141,1973.

Gillespie, J.S., Hamilton, D.N.H. \& Hosie, R.J.A.: The extraneuronal uptake and localization of noradrenaline in the cat spleen and the effect on this of some drugs of cold and of denervation. J. Physiol. 206:563-590, 1970 .

Gillespie, J.S. \& Towart, R.: Uptake kinetics and ion requirements for extraneuronal uptake of noradrenaline by arterial smooth muscle and collagen. Brit. J. Pharmacol. 47:566-567, 1973.

Glassman, A.H., Hurwic, M.J. \& Perel, J.M.: Plasma binding of imipramine and clinical outcome. Amer. J. Psychiatr. 130: $1367-1369,1973$.

Glassman, A.H., Perel, J.M., Shostak, M., Kantor, S.J. \& Fleiss, J.I.: Clinical implications of imipramine plasma levels for depressive illness. Arch. Gen. Psychiatr. 34:197-204,1977.

Glick, N.B.: Inhibitors of transport reactions. A. Inhibitors of ATP-ase: NaK-ATPase and related enzymic activities. In Metabolic Inhibitors Hochester, R.M., Kates, M. \& Quastel, J.H. (eds.) Academic Press, New York. $3: 1-45,1972$.

Glowinsky, J. \& Iversen, I.I.: Regional studies of catechol amines in the rat brain. J. Neurochem. 13:655-669, 1966.

Goldstein, A.: Biostatistics: An Introductory Text 
MacMillan Co., New York. p.154,1967.

Goodlet, I., Mireylees, S.E. \& Sugrue, M.F.: Effects of mianserin, a new antidepressant, on the in-vitro and in-vivo uptake of monoamines. Brit. J. Pharmacol. 61: $307-313,1977$.

Graefe, H.-H.: Methodology of catecholamine transport studies: Definition of terms. In The Mechanism of Neuronal and Extraneuronal Transport of Catecholamines Paton, D.W. (ed.) Kaven Press, il ew York. 7-35,1976.

Grahame-Smith, D.G. \& Parfitt, A.H.: Tryptophan transport across the synaptosomal membrane. J. Neurochem. 17: $1339-1353,1970$.

Gray, E.G. \& Whittaker, V.P.: The isolation of nerve endings from brain: An electron-microscopic study of cell fragments derived by homogenization and centrifugation. J. Anat. 26:79-88,1962.

Guroff, G., King, W. and Udenfriend, S.: The uptake of tyrosine by rat brain in-vitro. J. Biol. Chem. 236: $1773-1777,1961$.

Guth, P.S.: Effect of phenothiazines on neurohumor-containing particles from mammalian brain. Fed. Proc.

(Amer. Soc. Exp. Biol.) 21:1100-1102,1962.

Haga, T. \& Noda, F.: Choline uptake systems of rat brain synaptosomes. Biochim. Biophys. Acta 291:564-575,1973.

Hamberger, B.: Reserpine-resistant uptake of catecholamines in isolated tissues of the rat. Acta Psysiol. Scand. 71Suppl.295:7-55,1967.

Hamberger, B., Malmfors, T., Norberg, K.A. and Sachs, C.: Uptake and accumulation of catecholamines in peripheral adrenergic neurons of reserpinized animals, studied with a histochemical method. Biochem. Pharmacol. 13: $841-844,1964$.

Hamberger, B. \& Masuoka, D.: Localization of catecholamine uptake in rat brain slice. Acta Pharmacol. Toxicol. $\underline{22}: 363-368,1965$.

Hammer, W., Idestrom, C.M. \& Sjoqvist, F.: Clinical control of antidepressant drug therapy. In Antidepressant Drugs Garattini, S. \& Dukes, M.N.G. (eds.) Excerpta Medica Foundation, International Congress Series No. 122. 301-310,1966.

Harper, A.M., Duewer, D.I., Kowalski, B.R. \& Fasching, J.I.: ARTHUR and experimental data analysis: The use of poly- 
algorithm. In Chemometric Theory and Applications American Chemical Society Symposium Series No. 52 , 1977.

Harris, J.E. \& Baldessarini, R.J.: The uptake of $\mathrm{H}^{3}-$ dopamine by homogenates of rat corpus striatum: Effects of cations. Iife Sci. 13:303-312,1973.

Heilbronn, E.: The effect of phospholipases on the uptake of atropine and acetylcholine by slices of mouse brain cortex. J. Neurochem. 16:627-635,1969.

Hemsworth, B.A. \& Bosman, H.B.: The incorporation of triethylcholine into isolated guinea pig cerebral cortex synaptosomal and synaptic vesicle fraction. Europ. J. Pharmacol. 16:164-170,1971.

Hendley, E.D. \& Snyder, S.H.: Relationship between the action of monoamine oxidase inhibitors on the noradrenaline uptake system and their antidepressant efficacy. Nature 220:1330-1331,1968.

Hertting, G., Potter, I.T. \& Axelrod, J.: Effect of decentralization and ganglionic blocking agents on the spontaneous and reserpine induced release of $\mathrm{E}^{3}$-norepinephrine. J. Pharmacol. 136:289-292,1962.

Hodgkin, A.I. \& Martin, K.: Choline uptake by giant axons of Loligo. J. Physiol. 179:26-27,1965.

Hokfelt, T., Jonsson, G. \& Lidbrink, P.: Plectron microscopic identification of nerve ending particles in rat brain homogenates. Brain Res. 22:147-151,1970.

Horn, A.S.: Structure-activity relations for the inhibition of catecholamine uptake into synaptosomes from noradrenaline and dopaminergic neurones in rat brain homogenates. Brit. J. Pharmacol. 47:332-338,1973.

Hornykiewicz, 0.: Dopamine (3-hydroxytryptamine) and brain function. Pharm. Rev. 18:925-964,1966.

Hosli, I. \& Hosli, E.: Autoradiographic localization of the uptake of glycine in cultures of rat medulla oblongata. Brain Res. 45:612-616,1972.

Hunt, P., Kannengiesser, M-Y. \& Raynaud, J-P.: Nomifensine: A new potent inhibitor of dopamine uptake into synaptosomes from rat brain corpus striatum. J. Pharm. Pharmaco1. 26:370-371,1974.

Inch, T.D., Green, D.M. \& Thompson, P.3.J.: J. Pharm. Pharmacol. 25:359,1973. 
Iversen, I.I.: The inhibition of noradrenaline uptake by drugs. Advan. Drug Res. 2:5-46,1965.

Iversen, I.I.: The uptake of adrenaline by the rat isolated heart. Brit. J. Pharmacol. 24:387-394,1965a

Iversen, I.I.: The uptake of catecholeamines at high perfusion concentrations in the rat isolated heart: $\mathrm{A}$ novel catecholeamine uptake process. Brit. J. Pharmacol. 25: $18-33,1965 \mathrm{~b}$.

Iversen, I.I.: Role of transmitter uptake mechanisms in synaptic neurotransmission. Brit. J. Pharmacol. 4l: 571-591,1971.

Iversen, I.I.: Methods involved in studies of the uptake of biogenic amines. In Methods in Ivestigative and Diagnostic Endocrinology Berson, S.A. (ed.) NorthHolland Fublishing Co. Amsterdam I:569-603,1972.

Iversen, I.I.: Uptake process for biogenic amines. In Handbook of Psychopharmacology Iversen, I.I., Iversen, S.D. \& Snyder, S.H. (eds.) 3:381-442,1975.

Iversen, I.I. \& Bloom, F.E.: Studies of the uptake of $\mathrm{H}^{3}$ GABA and $\mathrm{H}^{3}$-glycine in slices and homogenates of rat brain and spinal cord by electron microscopic autoradiography. Brain Res. 41:131-143,1972.

Iversen, I.I., Glowinski, J. \& Axelrod, J.: The physiological disposition and metabolism of norepinephrine in immunosympathectomized animals. J. Pharmacol. Exp. Ther. 151:273-284,1966.

Iversen, I.I. \&.Johnston, G.A.R.: GABA uptake in rat central nervous system: Comparison of uptake in slices and homogenates and the effects of some inhibitors. J. Neurochem. 18:1939-1950,1971.

Iversen, L.I. \& Neal, M.J.: The uptake of H3-GABA by slices of rat cerebral cortex. J. Neurochem. 15:1141-1149, 1968.

Iversen, I.I. \& Salt, P.J.: Inhibition of the extra-neuronal uptake of catecholamine in the isolated rat heart by cholesterol. Nature New Biol. 238:91-92,1972.

Iversen, I.I. \& Snyder, S.H.: Synaptosomes: Different populations storing catecholamines and gamma-aminobutyric acid in homogenates of rat brain. Nature 220:796-798; 1968.

Jacobowitz, D. \& Brus, R.: A study of the extraneuronal 
uptake of norepinephrine in the perfused heart of the guinea pig. Europ. J. Pharmacol. 15:274-284,1971.

Jacobsen, E.: The theoretical basis of the chemotherapy of depression. In Depression: Proceedings of the Symposium held at Cambridge, 22 to 26 Sept. 1959 Davies, E.. (ed.) Cambridge Univ. Press 208,1964.

Janowsky, D.S., El-Yousef, M.K., Davis, J.M. \& Sekerke, H.G.: A cholinergic-adrenergic hypothesis of mania and depression. Iancet ii $: 632-635,1972$.

Johnston, G.A.R. \& Iversen, I.I.: Glycine uptake in rat central nervous system slices and homogenates:

Evidence for different uptake systems in spinal cord and cerebral cortex. J. Neurochem. 18:1951-1961,1971.

Kaiser, Ed.: Personal Communication. Research Associate, Dept. Endocrinology, Rhode Island Fospital. Unpublished Doctoral Dissertation research.

Kamijo, K., Koelle, G.B. \& Wagner, H.H.: Modification of the effects of sympathomimetic amines and of adrenergic nerve stimulation by 1-isonicotinyl-2-isopropylhydrazine (IIH) and isonicotinic acid hydrazide (INH). J. Pharmacol. 117:213-227,1955.

Kant, G.J. \& Meyerhoff, J.I.: Release of endogenous norepinephrine from rat hypothalamus in-vitro. Iife Sci. 20:149-154,1977.

Katz, B. \& Miledi, R.: The binding of acetylcholine to receptors and its removal from the synaptic cleft. J. Physiol. 231:549-574,1973.

Katz, H.S., Salehmoghaddum, S. \& Collier, B.: The accumulation of radioactive acetylcholine by a sympathetic ganglion and by brain: Failure to label endogenous stores. J. Neurochem. 20:569-579,1973.

Katz, R.I. \& Chase, T.N.: Neurohumoral mechanisms in the brain slice. Advan. Pharmac. Chemother. 8:1-30,1970.

Kirshner, N.: Uptake of catecholamines by a particulate fraction of the adrenal medulla. J. Biol. Chem. 237:2311-2317, 1962 .

Klawans, H.I.: The pharmacology of Parkinsonism. Dis. Nerv. Syst. $29: 805-816,1968$.

Koelle, G.B. \& Valk, A.: Physiological implications of the histochemical localization of monoamine oxidase. J. Physiol. 126:434-447,1954. 
Kopin, I.J., Gordon, E.K. \& Horst, D.: Determinants of tissue uptake of norepinephrine. Fed. Proc. (Amer. Soc. Exp. Biol.) 23:349,1964.

Kuhar, M.J.: Neurotransmitter uptake: A tool in identifying neurotransmitter specific pathways. Life Sci. $13: 1623-1634,1973$.

Kuhar, M.J., Aghajanian, G.K. \& Roth, R.H.: Serotonin neurons: A synaptic mechanism for the reuptake of serotonin. In Advances in Biochemical Psychooharmacology Raven Press, New York. 10:287-295, 1974 .

Kuhar, M.J., Green, A.I., Snyder, S.H. \& Gfeller, E.: Separation of synaptosomes storing catecholamines and gamma-aminobutyric acid in rat corpus striatum. Brain Res. 21:405-417,1970.

Kuhar, M.J., Roth, R.H. \& Aghajanian, G.K.: Synaptosomes from forebrains of rats with mid brain raphe lesions: Selective reduction of serotonin uptake. J. Pharmacol. Exp. Ther. 181:36-45,1972.

Kuhar, M.J., Sethy, V.H., Roth, R.H. \& Aghajanian, G.K.: Choline: Selective accumulation by central cholinergic neurons. J. Neurochem. 20:581-593,1973.

Kuriyama, K., Weinstein, H. \& Roberts, E.: Uptake of gammaaminobutyric acid by mitochondrial and synaptosomal fractions from mouse brain. Brain Res. 16:479-492, 1969.

Laduron, P.: Tissue fractionation in neurobiochemistry: An analytical tool or a source of artifacts. Int. Rev. Neurobiol. 251-281, 1977.

Lane, J.D. \& Aprison, M.H.: Calcium-dependent release of endogenous serotonin, dopamine and norepinephrine from nerve endings. Iife Sci. 20:665-672,1977.

Langer, S.Z.: The metabolism of $\mathrm{H}^{3}$-noradrenaline released by electrical stimulation from the isolated nictitating membrane of the cat and from the vas deferens of the rat. J. Physiol. 208:515-546,1970.

Iapin, I.P. \& Oxenkrug, G.F.: Intensification of the central serotoninergic processes as a possible determinant of the thymoleptic effect. Lancet $1: 132-136,1969$.

Ieonard, B.E.: Neurochemical and neuropharmacological aspects of depression. Int. Rev. Neurobiol. 18:357-387, 1975.

Levi, G. \& Raiteri, M.: Detectability of high and low 
affinity uptake systems for GABA and glutamate in rat brain slices and synaptosomes. Iife Sci. 12:81-88, 1973a.

Levi, G. \& Raiteri, M.: GABA and glutamate uptake by subcellular fractions enriched in synaptosames: Critical evaluation of some methodological aspects. Brain Res. $165-185,1973 b$.

Levi, G. and Raiteri, M.: Synaptosomal transport processes. Int. Rev. Neurobiol. 51-74,1976.

Lew, G.M. \& Quay, W.B.: Twenty four hour rhythmic uptake of $\mathrm{H}^{3}$-norepinephrine in vitro by hypothalamus and medial lower brainstem. Int. J. Chronobiol. 2:209-213,1974.

Iiang, C.C. \& Quastel, J.H.: Uptake of acetylcholine in rat brain cortex slices. Biochem. Pharmacol. 18:1169-1185, 1969.

Lidbrink, P. \& Jonsson, G.: Noradrenaline nerve terminals in the cerebral cortex: Effects on noradrenaline uptake and storage following axonal lesion with 6-hydroxydopamine. J. Neurochem. 22:617-626,1974.

Iightman, S. \& Iversen, I.I.: Role of uptake, in the extraneuronal uptake and metabolism of catecholamines in the isolated rat heart. Brit. J. Pharmacol. 37:638-649,1969.

Iindmar, R. \& Muscholl, D.: Die wirkung von pharmaka auf die elimination von noradrenalin aus der perfusions flussigkeit und die noradrenalin-aufnahme in das isolierte herz. Arch. Exp. Path. Pharmakol. 247: 469-472, 1964 .

Iineweaver, F. \& Burk, D.: The determination of enzyme dissociation constants. J. Amer. Chem. Soc. 56: 658-666, 1934 .

Ijungdahl, A. \& Hokfelt $\mathrm{T}$.: Autoradiographic uptake patterns of $(3 \mathrm{H}) \mathrm{GABA}$ and $(3 \mathrm{H})$ glycine in central nervous tissue with special reference to the cat spinal cord. Brain Res. 62:587-595,1973.

Iogan, W.J. \& Snyder, S.H.: High affinity uptake systems for glycine, glutamic acid and aspartic acid in synaptosomes of rat central nervous tissue. 3rain Res. 42:413-431,1972.

Lowry, O.H., Rosebrough, I.J., Farr, A.I. \& Randall, R.J.: Protein measurement with the folin phenol reagent. J. Biol. Chem. 193:265-275,1951.

Macmillan, W.H.: A hypothesis concerning the effects of 
cocaine on the action of sympathomimetic amines. Brit. J. Pharmacol. 14:385-391, 1959 .

Marchbanks, R.M.: Exchangeability of radioactive acetylcholine of synaptosomes and synaptic vesicles.

Biochem. J. 106:87-95, 1968a.

Marchbanks, R.M.: The uptake of $\left(\mathrm{C}^{14}\right)$ choline into synaptosomes in-vitro. Biochem. J. 110:533-541,1968b.

Marchbanks, R.M.: Biochemical organization of cholinergic nerve terminals in the cerebral cortex. In Cellular Dynamics of the Neuron Barondes, S.H. (ed.) Academic Press, New York 115-135, 1969.

Martell, A.E. \& Sillen, I.G.: Stability Constants Iondon Chemical Society, 1964; and Suppl. 1, 1971.

Massari, V.J. and Sanders-Bush, E.: Synaptosomal uptake and levels of serotonin in rat brain areas after parachloroamphetamine or B-9 lesions. Furop. J. Pharmacol. 33:419-422, 1975 .

Matus, A.I. \& Dennison, M.E.: An autoradiographic study of uptake of exogenous glycine by vertebrate spinal cord slices in-vitro. J. Neurocytol. 1:27-34,1972.

McClure, D.J.: The role of dopamine in depression. Can. Psychiatr. Assoc. J. 18:309-312,1973.

Mirkin, B.I., Giarman, N.J. and Freedman, D.X.: Uptake of noradrenaline by subcellular particles in homogenates of rat brain. Biochem. Pharmacol. 12:214-216,1963.

Muscettola, G., Goodwin, F.X., Potter, W.z., Claeys, M.M. \& Markey, S.P.: Imipramine and desipramine in plasma and spinal fluid. Arch. Gen. Psychiatr. 35:621-625, 1978.

Muscholl, E.: Die hemmung der noradrenalin-aufnahme des herzens durch reserpin und die wirkung von tyramin. Arch. Bxp. Path. Pharmakol. 240:231-234,1960.

Muscholl, E.: Effect of cocaine and related drugs on the uptake of noradrenaline by heart and spleen. Brit. J. Pharmacol. 16:352-359,1961.

Neal, M.J.: The uptake of $\left(\mathrm{C}^{14}\right)$ glycine by slices of mammalian spinal cord. J. Physiol. 215:103-117,1971.

Neame, K.D.: Phenylalanine as inhibitor of transport of amino acids in brain. Nature 192:173-174,1961.

Neame, K.D.: Uptake of histidine, histamine and other imida- 
zole derivatives by brain slices. J. Neurochem. 11: $655-662,1964$.

Norberg, K-A. \& Hamberger, $\mathrm{B}_{\text {.: }}$ The sympathetic adrenergic neuron. Acta Physio1. Scand. 63Suppl.238:5-42,1964.

O'Donnell, S.R. \& Saar, N.: A histochemical study of noradrenaline in the guinea pig trachea. Brit. J. Pharmacol. 49:267-278,1973.

Oswald, I., Brezinova, V. \& Dunleavy, D.I.F.: On the slowness of action of tricyclic antidepressant drugs. Brit. J. Psychiatr. 120:673-677,1972.

Parfitt, A. \& Grahame-Smith, D.G.: The transfer of tryptophan across the synaptosomal membrane. In Aromatic Amino Acid in the Brain Ciba Foundation Symposium lo. 22 Elsevier, New York 175-192,1974.

Peterson, N.A. \& Rughupathy, E.: Developmental transitions in uptake of amino acids by synaptosomal fractions isolated from rat cerebral cortex. J. Neurochem. 21: 97-110,1973.

Phillis, J.W.: Minireview: The role of calcium in the central effects of biogenic amines. Iife Sci. 14:11891201,1974 .

Polak, R.I.: The influence of drugs on the uptake of acetylcholine by slices of cerebral rat cortex. Brit. J. Pharmacol. 36:144-152,1969.

Polak, R.I., \& Meeuws, M.M.: The influence of atropine on the release and uptake of acetylcholine by the isolated cerebral cortex of the rat. Biochem. Pharmacol. 15: 989-992,1966.

Porter, W.R. \& Trager, W.F.: Improved non-parametric statistical methods for the estimation of MichaelisMenten kinetic parameters by the direct linear plot. Biochem. J. 161:293-302,1977.

Potter, I.T.: Uptake of choline by nerve endings isolated from rat cerebral cortex. In The Interactions of Drugs and Subcellular Components on Animal Cells Campbell, P.N. (ed.) 293-304,1968.

Potter, I.T. \& Axelrod, J.: Intracellular localization of catecholamines in tissues of the rat. Nature 194: $581-582,1962$.

Potter, I.T. \& Axelrod, J.: Subcellular localization of catecholamines in tissues of the rat. J. Pharmacol. $142: 291-298,1963$. 
Prange, A.J. Jr., Wilson, I.C., Lynn, C.W., Alltop, I.B. \& Stikeleather, R.A.: I-Tryptophan in mania. Arch. Gen. Psychiatr. 30:56-62,1974.

Raab, W. \& Humphreys, R.J.: Drug action upon myooardial epinephrine-sympathin concentration and heart rate. J. Pharmacol. Exp. Ther. 89:64-76,1947.

Rajan, K. S., Wiehle, R. D., Riessen, W. H., Colbum, R. W. \& Davis, J. M.: Effect of metal chelating agents on the storage of norpeinephrine in-vitro by cerebral synaptic vesicles. Biochem. Pharmacol. 26:1703-1708,1977.

Roberts, D. T.: Enzyme Kinetics. Cambridge University Press, New York 1-326,1977.

Rosell, S., Kopin, I.J. \& Axelrod, J.: Fate of $\mathrm{H}^{3}$-noradrenaline in sketal muscle before and following sympathetic stimulation. Am. J. Physiol. 205:317-321, 1963.

Ross, S.B. \& Renyi, A. I.: Blocking action of sympathomimetic amines on the uptake of tritiated noradrenaline by mouse cerebral cortex tissues in-vitro. Acta. Pharmacol. Toxicol. 2l:226-239,1964.

Ross, S.B. \& Renyi, A. I.: Uptake of some tritiated sympathomimetic amines by mouse brain cortex slices in-vitro. Acta. Pharmacol. Toxicol. 24:297-309,1966.

Ross, S. B. \& Renyi, A. L.: Inhibition of the uptake of tritiated catecholamines by antidepressant and related agents. Europ. J. Pharmacol. 2:181-186,1967a.

Ross, S. 3. \& Renyi, A. I.: Tricyclic antidepressant agents. II. Effect of oral administration on the uptake of $\mathrm{F}^{3}$ noradrenaline and $C^{14-5-h y d r o x y t r y p t a m i n e ~ i n ~ s l i c e s ~ o f ~}$ the midbrain-hypothalamos region of the rat. Acta Pharmacol. Toxicol. 36:395-408,1975b.

Roth, J.A. \& Gillis, C.I.: Some structural requirements for inhibition of type $A$ and $B$ forms of rabbit monoamine oxidase by tricyclic psychoactive drugs. Mol. Pharmacol. 11:28-35,1974.

Rubin, R.P.: The role of calcium in the release of neurotransmitter substances and hormones. Pharm. Rev. 22: 389-428, 1970 .

Salt, P.J.: Inhibition of noradrenaline uptake, in the isolated rat heart by steroids, clonidine and methoxylated phenylethylamines. Europ. J. Pharmacol. 20: $329-340,1972$. 
Sayers, A.C. \& Burki, H.R.: Antiacetylcholine activities of psychoactive drugs: A comparison of the (3H) quinuclidinyl benzilate binding assay with conventional methods. J. Pharm. Pharmacol. 28:252-253,1976.

Schildkraut, J.J: The catecholamine hypothesis of affective disorders: A review of supporting evidence. Amer. $J$. Psychiatr. 112:509-522, 1965 .

Schildkraut, J.J.: Norepinephrine metabolites as biochemical criteria for classifying depressive disorders and predicting responses to treatment: Preliminary findings. Amer. J. Psychiatr. 130:695-698, 1973.

Schildkraut, J.J. \& Kety, S.S.: Biogenic amines and emotion. Science 156:21-30,1967.

Schmidt, H.S., Clark, R.W. \& Hyman, P.R.: Protriptyline: An agent effective in the treatment of the narcolepsycataplexy syndrome and hypersomnia. Amer. J. Psychiatr. 134 : $183-185,1977$.

Schuberth, J.: Choline acetyltransferase: Furification and effects of salts on the mechanism of the enzyme catalysed reaction. Biochim. Biophys..Acta 122:470-481,1966.

Schuberth, J. \& Sundwall, A.: Effect of some drugs on the uptake of acetylcholine in cortex slices of mouse brain. J. Neurochem. 14:807-812,1967.

Shaskan, E.G. \& Snyder, S.H.: Kinetics of serotonin accumulation into slices from rat brain: Relationship to catecholamine uptake. J. Pharmacol. Exp. Ther. $175: 404-418,1970$.

Shein, H. \& Smith, S.E.: Structure-activity relationships for the anticholinoceptor action of tricyclic antidepressants. Brit. J. Pharmacol. 62:567-571,1978.

Shore, P.A.: Release of serotonin and catecholamines by drugs. Pharm. Rev. 14:531-550,1962.

Sjoqvist, F., Taylor, P.W. Jr. \& Titus, E.: The effects of immunosympathectomy on the retention and metabolism of noradrenaline. Acta Physiol. Scand. 69:13-22,1967.

Snodgrass, S.R., Hedley-Whyte, E.T. and Iorenzo, A.V.: GABA transport by nerve ending fractions of cat brain. J. Neurochem. 20:771-782,1973.

Snyder, S.H., Brown, B. \& Kuhar, M.J.: The synaptosomal localization of histamine, histadine decarboxylase, the histamine methyltransferase in rat hypothalamus. 
J. Neurochem. 23:37-45,1974.

Snyder, S.H. \& Coyle, J.T.: Regional differences in H3norepinephrine and $\mathrm{H}^{3}$-dopamine uptake into brain homogenates. J. Pharmacol. Exp. Ther. 165:78-86,1969.

Snyder, S.H., Glowinski, J. \& Axelrod, J.: The storage of norepinephrine and some of its derivatives in brain synaptosomes. Life Sci. $4: 797-807,1965$.

Snyder, S.H., Green, A.I. \& Hendley, E.D.: Kinetics of $\mathrm{Y}^{3}$ norepinephrine accumulation into slices from different regions of the rat brain. J. Pharmacol. Exp. Ther. $164: 90-102,1968$.

Snyder, S.H., Iogan, W.J., Bennett, J.P. \& Arregui, A.: Amino acids as central nervous transmitters: Biochemical studies. In Neuroscience Research: Chemical Aporoaches to Brain Function Fhrenpreis, S. 2 Kopin, I.J. (eds.) Academic rress, New York 131-157, 1973.

Snyder, S.H. \& Yaminamura, H.I.: Antidepressants and the muscarinic acetylcholine receptor. Archiv. Gen. Psychiatr. 34:236-239,1977.

Stromblad, B.C.R. \& Nickerson, M.: Accumulation of epinephrine and norepinephrine by some rat tissues. J. Pharmacol. Exp. Ther. 134:154-159,1961.

Thoenen, H., Hurlimann, A. \& Haefely, W.: The effect of sympathetic nerve stimulation on volume, vascular resistance and norepinephrine output in the isolated perfused spleen of the cat and its modification by cocaine. J. Pharmacol. Exp. Ther. 143:57-63,1964a.

Thoenen, H., Hurlimann, A. \& Haefely, W.: Wirkungen von phenoxybenzamin, phentolamin und azapetin auf adrenergische synapse der katzenmilz. Helv. Physiol. Pharmacol. Ácta 22:148-161,1964b.

Tipton, K.F., Mantle, T.J. \& Houslay, M.D.: A kinetic method for the detection of inhibitory contaminants in radioactive substrates. Biochem. Pharmacol. 26: $1525-1527,1977$.

Tissari, A.H. \& Bogdanski, D.F.: Biogenic amine transport, VI. Comparison of effects of ouabain and $\mathrm{K}^{+}$deficiency on the transport of 5-hydroxytryptamine and norepinephrine by synaptosomes. Pharmacology 5:225-234,1971.

Tseng, I. F. \& Ioh, H. H.: Significance of dopamine receptor activity in methoxy-amphetamine and d-amphetamineinduced locomotor activity. J. Pharmacol. Exp. Ther. $189: 717-724,1974$ 
Tseng, I. F., Hitzemann, R. J. \& Ioh, H. H.: Comparative effects of $d, 1-p-m e t h o x y$-amphetamine and d-amphetamine on catecholamine release and reuptake in-vitro. J. Pharmacol. Exp. Ther. 189:708-716,1974.

Umbreit, W. W., Burris, R. H. \& Stauffer, J. F.: Manometric Techniques Fourth Edition Burgess Publ. Co. 132,1964.

Weil-Malherbe, H.: The biochemistry of affective disorders. In Handbook of Neurochemistry: Pathological Chemistry of the Nervous System. Rlenum Press, New York 371-416, 1972.

Wentworth, i. E.: Rigorous least squares adjustment: Application to some non-linear equations, I. J. Chem. Ed. 42: 96-103,1965(a).

Wentworth, W. E.: Rigorous least squares adjustment: Applications to some non-linear equations, II. J. Chem. Ed. 42: $162-167,1965(\mathrm{~b})$.

Whitby I. G., Axelrod, J. \& Weil-Vialherbe, H.: The fate of $\mathrm{H}^{3}$-norepinephrine in animals. J. Pharmacol. Exp. Ther. 132:193-201,1961.

White, + T. D. \& Keen, P.: Effects of inhibitors of $\left(\mathrm{Na}^{+}+\right.$ $\left.\mathrm{K}^{+}\right)$-dependent adenosine triphosphatase on the uptake of norepinephrine by synaptosomes. Mol. Pharmacol. I: 4045,1971 .

Wilkerson, R.D.: Antiarrhythmic effects of tricyclic antidepressant drugs in ouabain induced arrhythmic in the dog. J. Pharmacol. Bxp. Ther. 205(3):666-674,1978.

Wilkinson, G.N.: Statistical estimations in enzyme kinetics. Biochem. J. 80:324-332,1961.

Winer, B. J.: Statistical Principles in Experimental Design Second Edition Pickraw-Hill, Inc., New York 51-54,1971.

Wofsey, A.R., Kuhar, M. J. \& Snyder, S.H.: A unique synaptosomal fraction which accumulates glutamic and aspartic acids in brain tissue. Proc.NatI. Acad. Sci. 68:1102$1106,1971$.

Wolfe, D. E., Potter, I. T. and Webster, E. I.: Radioantographic localization of norepinephrine- $\mathrm{H}$ in the tissues of mice. J. Pharmacol. Exp. Ther. 138:376-381,1962.

Wong, D. T., Horng, J. S. and Fuller, R. W.: Kinetics of serotonin accumulation into synaptosomes of rat braineffects of amphetamine and chloroamphetamines. Biochem. 
Pharmacol. 22:311-322,1973.

Wurtman, R. J. \& Axelrod, J.: A sensitive and specific assay for the estimation of monoamine oxidase. Biochem. Pharmacol. 12:1439-1441,1963.

Wylie, D. N., Archer, S. \& Arnold, A.: Augmentation of pharmacological properties of catecholamines by $0-$ methyl transferase inhibitors. J. Pharmacol. 130:239244,1960 .

Yamamurs, H. I. \& Snyder, S. H.: High affinity transport of choline into synaptosomes of rat brain. J. Neurochem. 21:1355-1374,1973.

Yoshida, H., Namba, J., Kaniike, K. \& Imaizumi, R.: Studies on the active transport of l-dopa (dihydroxy-phenylalanine) into brain slices. Jap. J. Pharmacol. 13: 1$9,1963 a$.

Yoshida, H., Kanilke, K. \& Namba, J.: Inhibitions by ouabain and protoveratrine on active transport of 1-dopa into brain slices. Jap. J. Pharmacol. 13:10-17,1963b.

Yoshida, H., Kaniike, K. \& Namba, J.: Properties of a carrier system to transport 1-dopa into brain slices. Nature 198(4876): 191-192,1963c.

Yoshida, H., Fujisawa, H. \& Ohi, Y.: Influences of protanine on the $\mathrm{Na}^{+}, \mathrm{K}^{+}$-dependent $\mathrm{ATP}$-ase and on the active transport processes of potassium and of l-dopa into brain slices. Can.J. Biochem. 43:841-849,1965.

Young, A. B., Pert, C. D., Brown, D. G., Taylor, K. M. \& Snyder, S.H.: Nuclear localization of histamine in neonatal rat brain. Science 173:247-249,1971.

Zaimis, E., Berk, I. \& Callingham, B.: Morphological biochemical and functional changes in the sympathetic nervous system of rats treated with NGF-antiserum. Nature 206:1221-1222,1965.

Zigmond, M. J., Chalmers, J. P., Simpson, J. R. \& Wurtman, R. J.: Effect of lateral hypothalamic lesions on uptake of norepinephrine by brain homogenates. J. Pharmacol. Fxp. Ther. 179:20-28,1971. 
137

APPFIDIX 
Figure A-I. Flowchart for "VIVO": A program used to calculate data for synaptosomal uptake studies

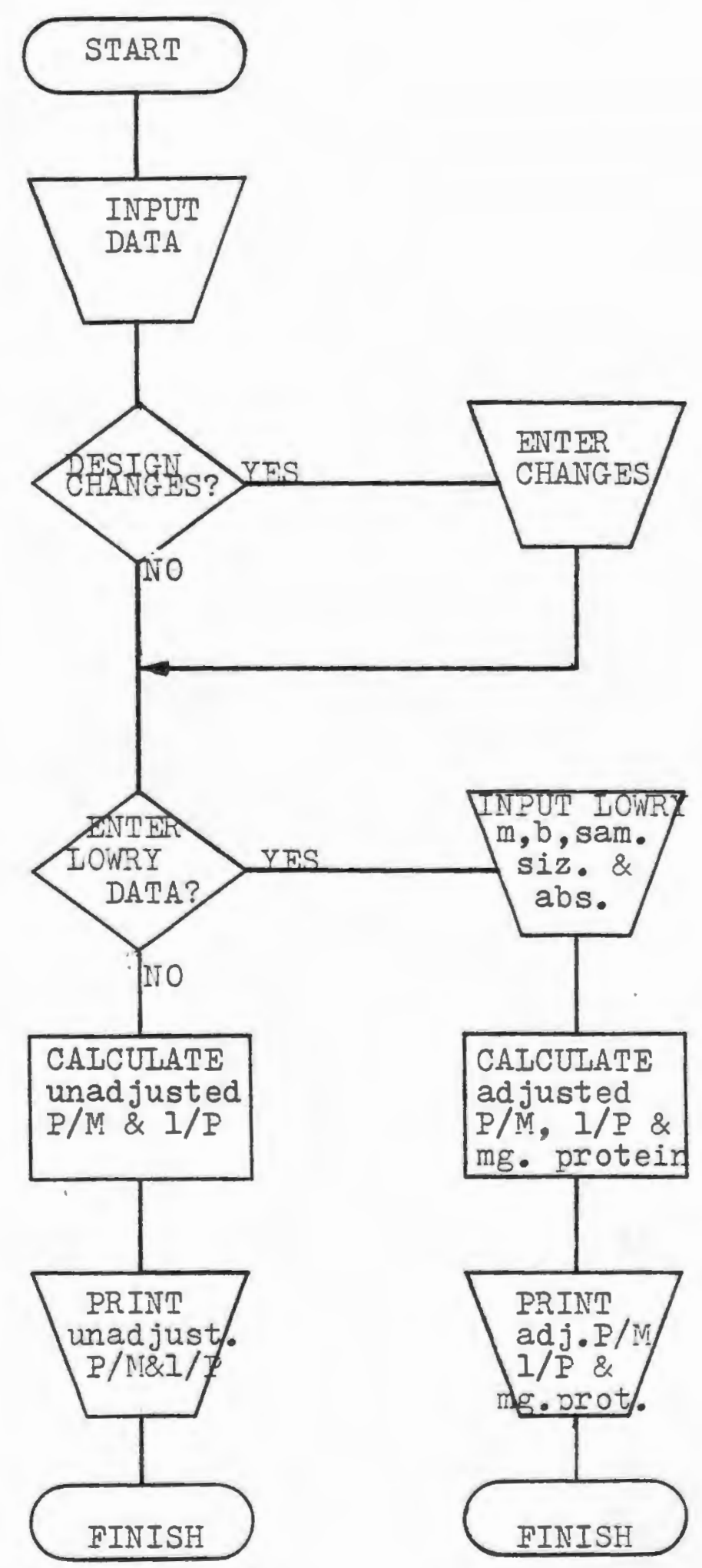


Table A-1. Iisting of 'VIVO': A program used to calculate data for synaptosomal uptake studies.

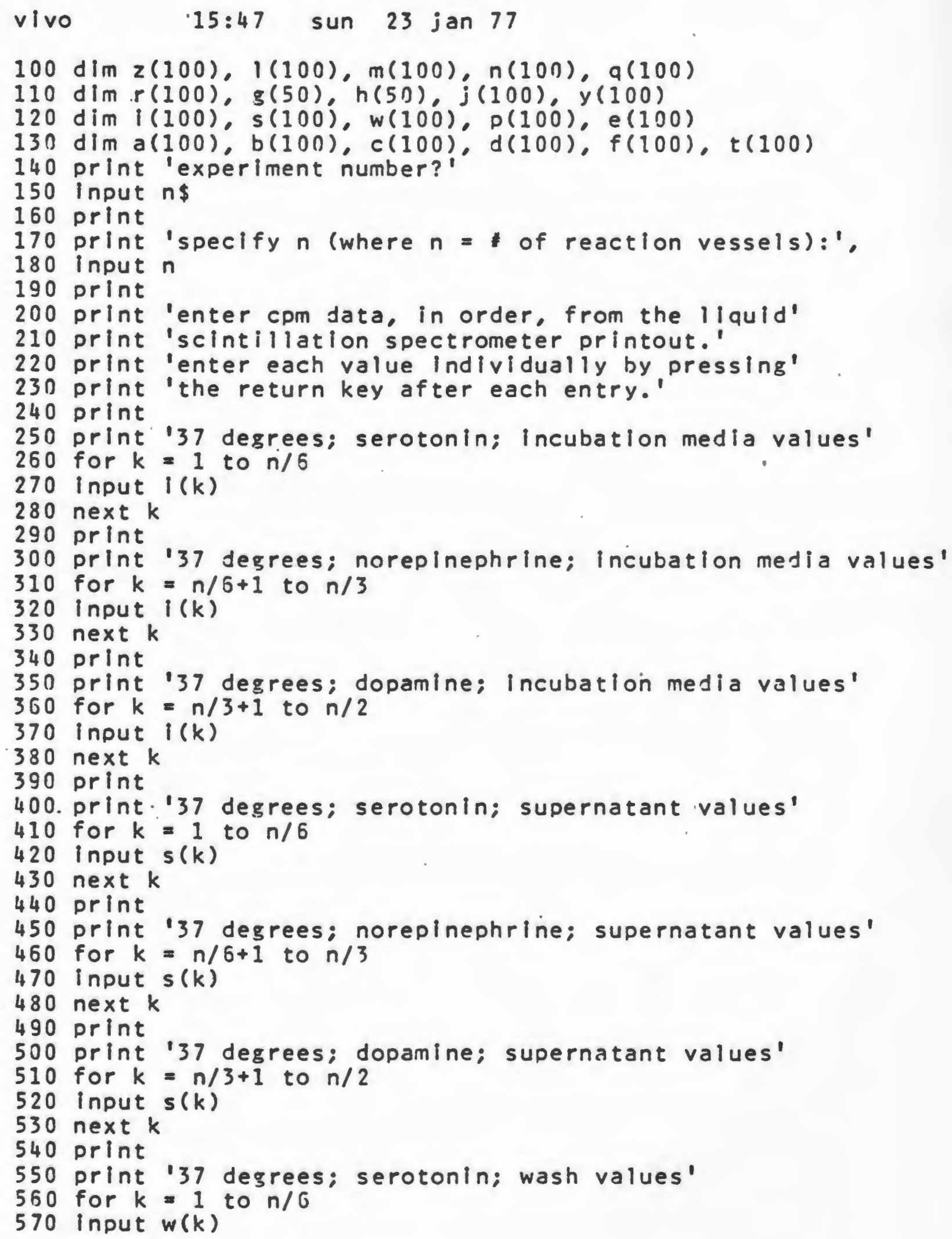




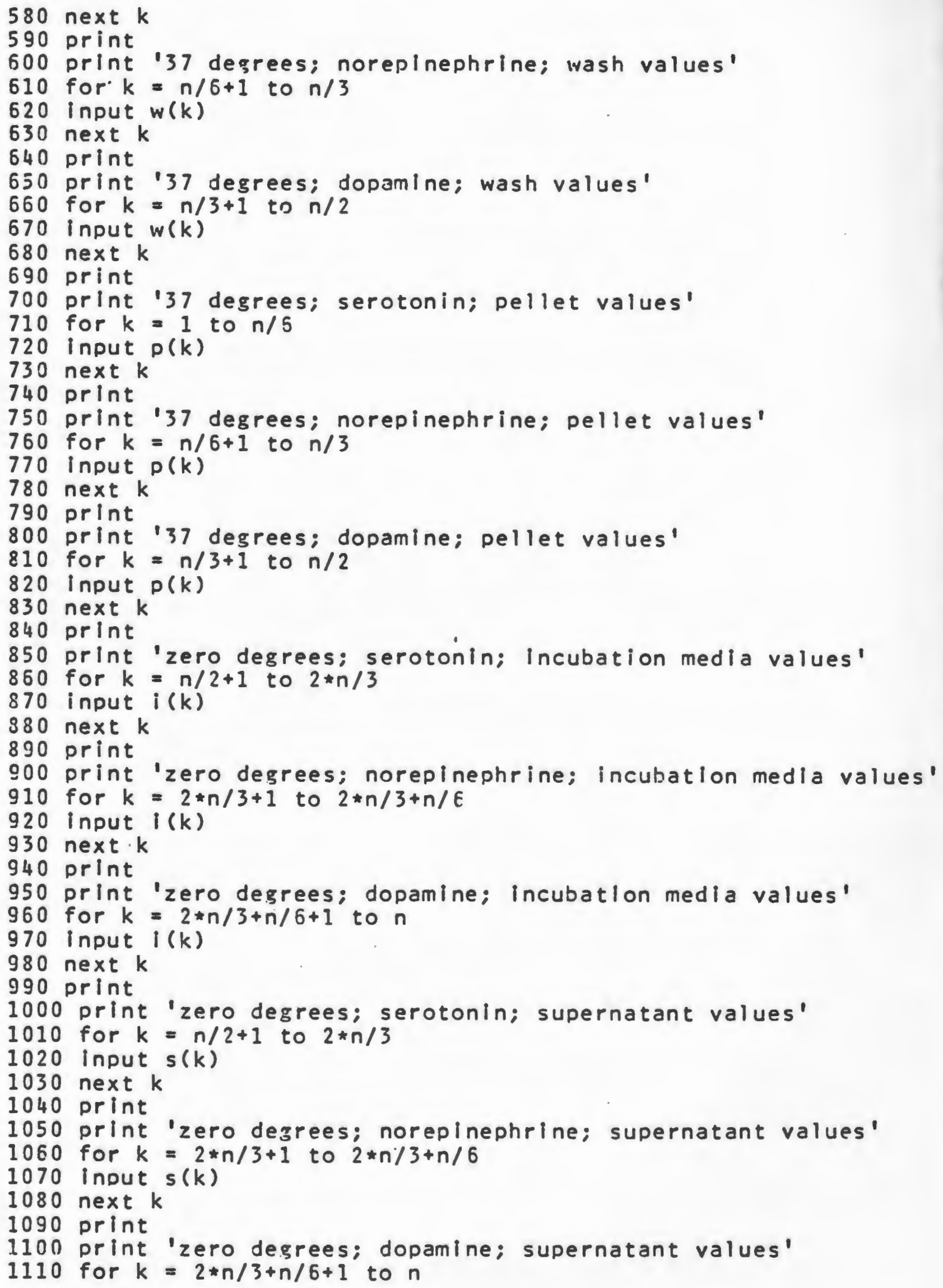




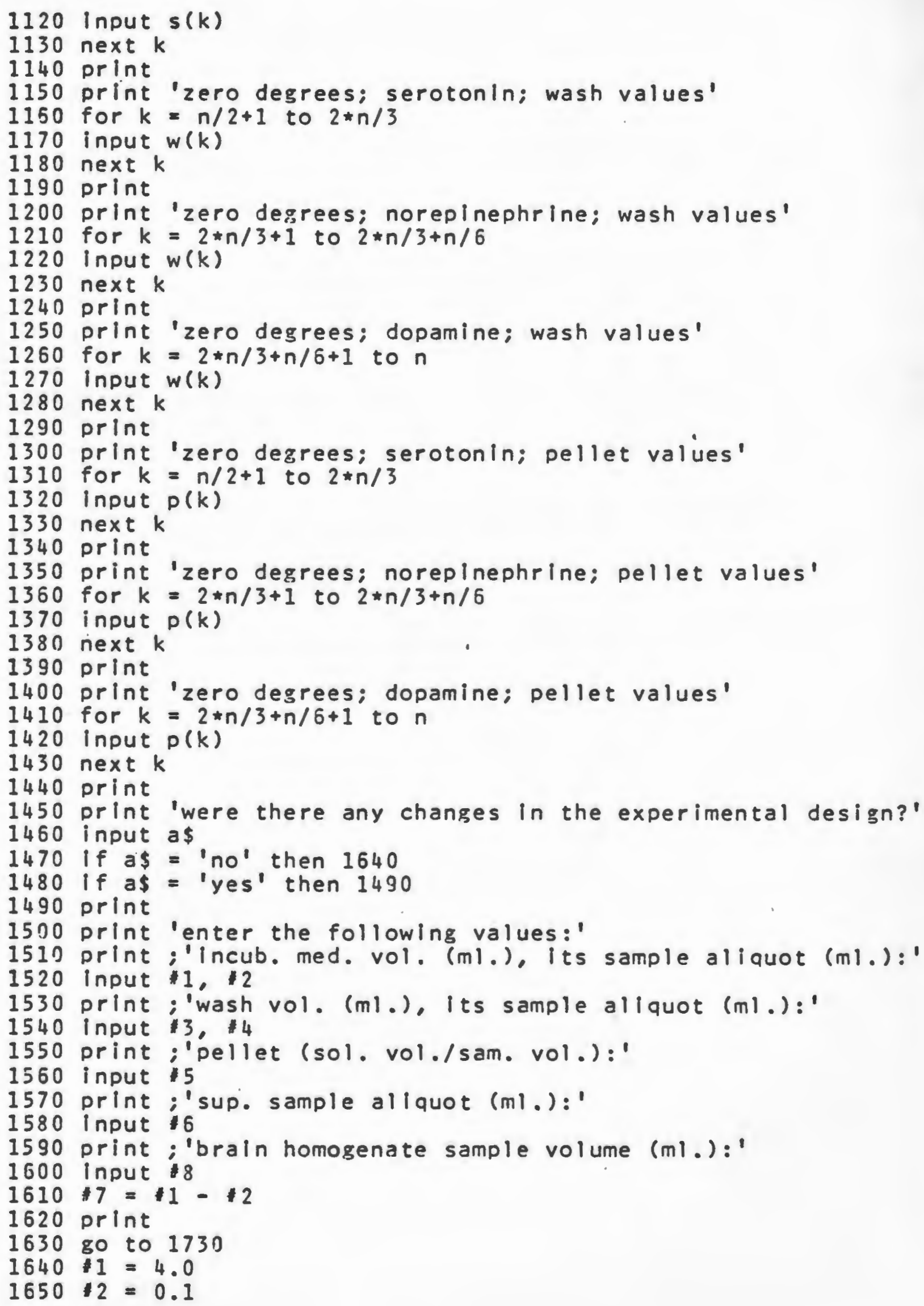




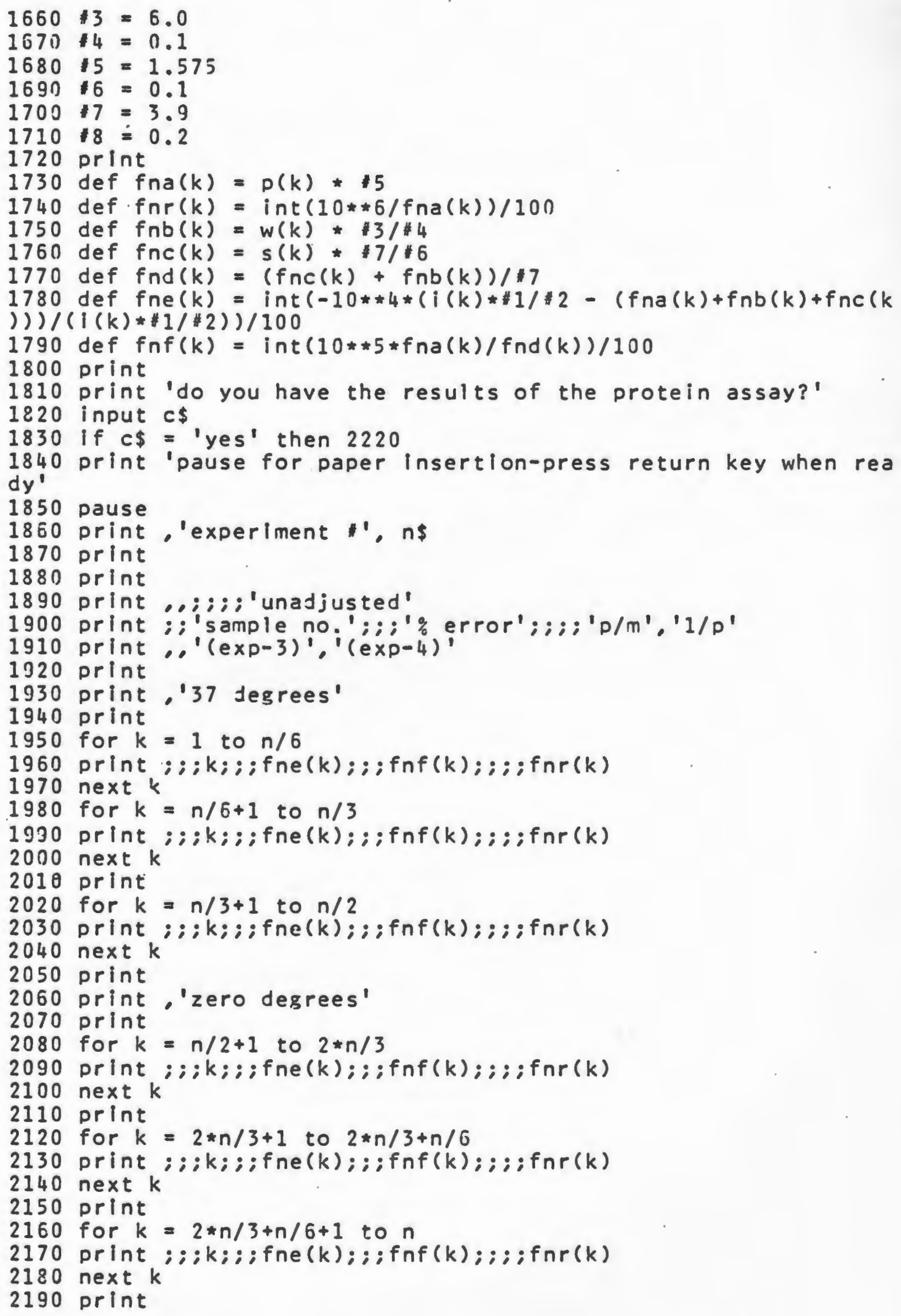


2200 go to 3410

2210 print

2220 print

2230 print

2240 print

'enter the results of the protein assay in the form'

$'(m, b, a 1, s 1, a 2, s 2 \ldots . . a n, s n)$ where $m$ and $b$ are the'

ively: '

2250 print 'al to an are the absorbances and $s 1$ to sn are their respective'

$22 \in 0$ print 'sample sizes $(\mathrm{ml}$.$) used to obtain valld absorbance$ readings ${ }^{\prime}$

2270 print 'beginning with $1=$ forebrain control, $2=10$ dose, $3=1$ ow-med,'

2280 print ' $4=h i-m e d$ and $5=h i$ dose; $6-10$ represent hypothalamus control to'

2290 print 'hi dose samples and finally $11-15$ are striatal valu es.'

2300 print : ' '(enter 2 values per line)'

2310 print

2320 input $m, b$

2330 print

2340 for $k=1$ to $n / 2$

2350 input $g(k), h(k)$

2360 next $k$

2370 def $f n j(k)=1 g t(g(j))$

2330 def $f$ ny $(k)=(f n j(k)-b) / m$

$2390 \mathrm{dim} k(100)$

2400 def $f n k(k)=\operatorname{lnt}(1000 *($ fny $(k) * 18 / h(j) / 10 * * 3)) / 1000$

2410 def $f_{n l}(k)=f n a(k) / f n k(k)$

2420 def $f n m(k)=f n l(k) / f n d(k)$.

2430 def $f n n(k)=10 * \star 5 / f n l(k)$

2440 print 'pause for paper insertion-press return key when rea dy'

2450 pause

2460 print, 'experiment ', n\$

2470 print

2480 print

2490 print i; ; : lowry adjusted'

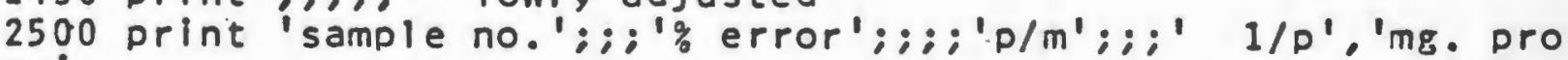
t.'

2510 print , $; ; i^{\prime} i^{\prime}(\exp -3)^{\prime} ; i^{\prime}(\exp -5)^{\prime}$

2520 def $f n a(k)=\operatorname{lnt}(10 * * 5 * f n m(k)) / 100$

2530 def $f n t(k)=\operatorname{int}(10 * * 2 * f n n(k)) / 100$

$2540 \mathrm{dim}$ o( 30$)$

$2550 \operatorname{dim} u(30)$

2560 print

2570 print, '37 degrees'

2580 print

2590 for $k=1$ to $n / 6$

2600 let $j=k$

2610 print ; $k$;:; fne $(k) ; ; ; f n q(k) ;: ; f n t(k): ; ; f n k(k)$

2620 let $o(k)=f n q(k)$

2630 let $u(k)=f n t(k)$

2640 next $k$

2650 print

2660 for $k=n / 5+1$ to $n / 3$

2670 let $j=k$ 


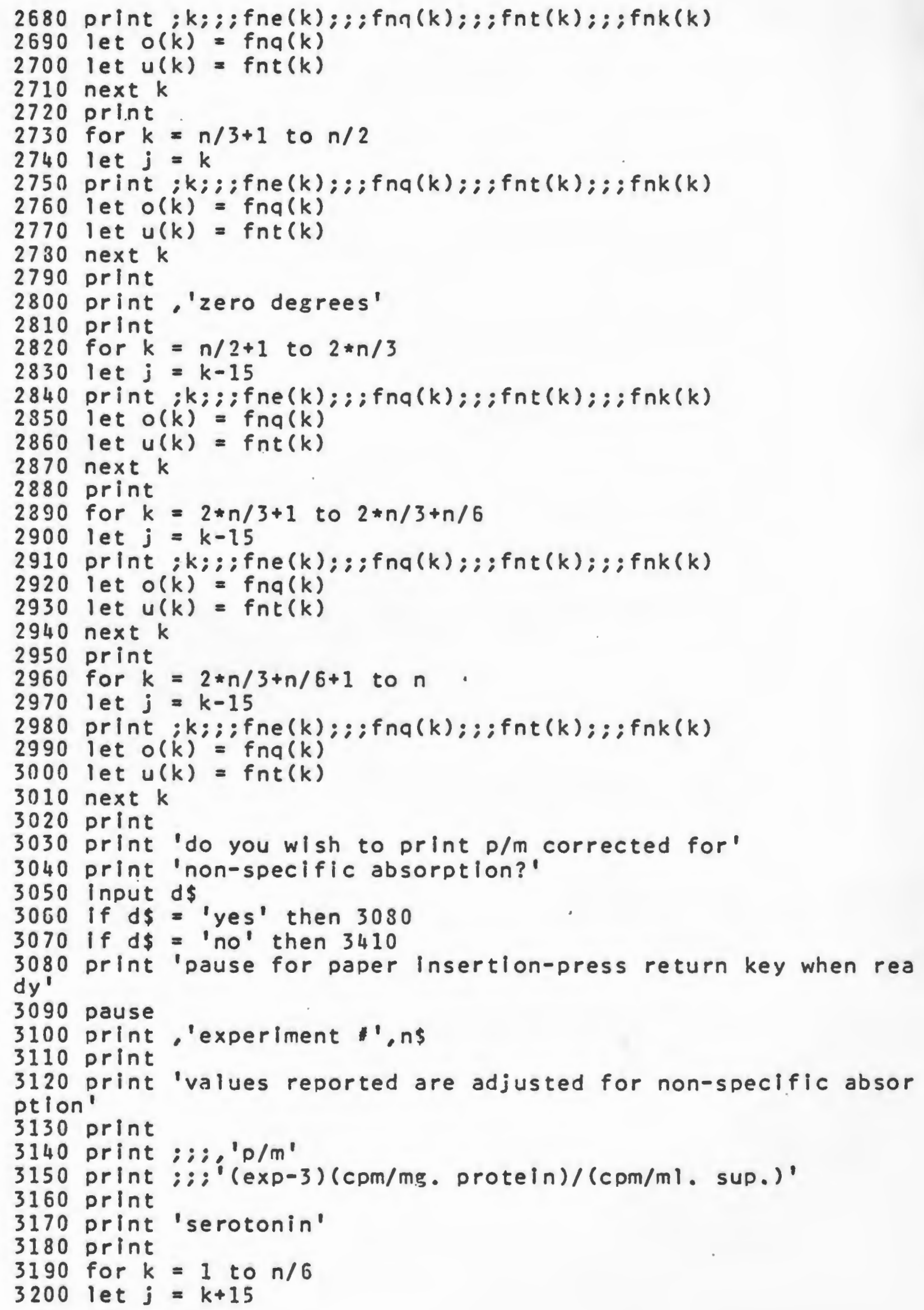




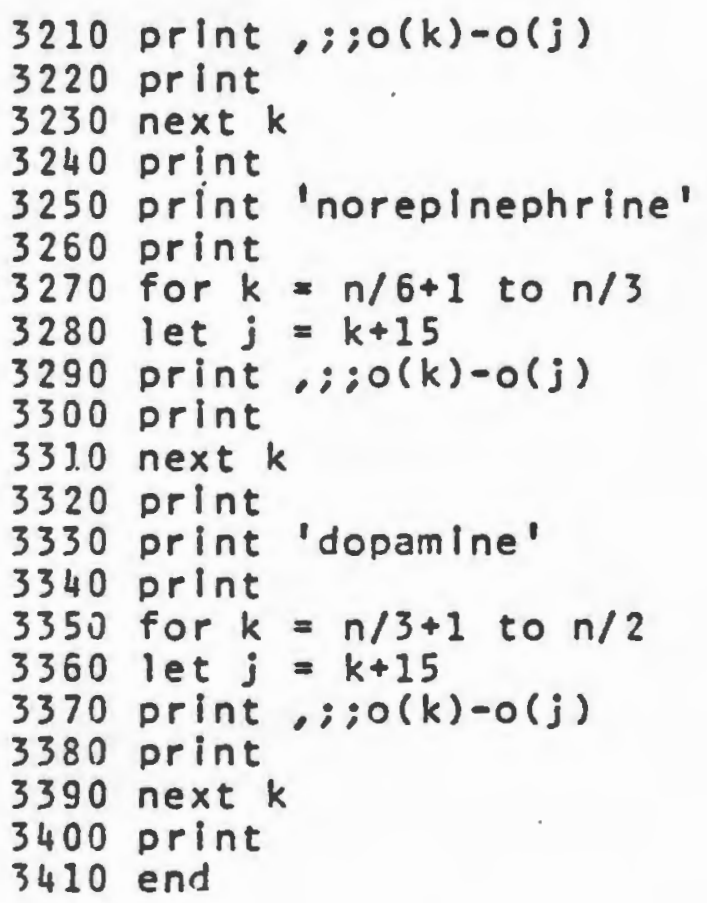

